Flavio Guimarães Caduda

\title{
Método de Alta Resolução em Imageamento Acústico
}

Dissertação apresentada à Escola Politécnica da Universidade de São Paulo para a obtenção do título de Mestre em Engenharia Elétrica

São Paulo 
Flavio Guimarães Caduda

Engenheiro Eletricista, Escola Politécnica da USP, 2003

\section{Método de Alta Resolução em Imageamento Acústico}

Dissertação apresentada à Escola Politécnica da Universidade de São Paulo para a obtenção do título de Mestre em Engenharia Elétrica

Área de Concentração:

Sistemas Eletrônicos

Orientador:

Luiz Antonio Baccalá

São Paulo 


\section{FICHA CATALOGRÁFICA}

\section{Caduda, Flavio Guimarães}

Método de alta resolução em imageamento acústico / F.G. Caduda. -- São Paulo, 2011.

$59 \mathrm{p}$.

Dissertação (Mestrado) - Escola Politécnica da Universidade de São Paulo. Departamento de Engenharia de Telecomunicações e Controle.

1. Processamento digital de sinais 2. Acústica 3. Estrutura de aeronaves I. Universidade de São Paulo. Escola Politécnica. Departamento de Engenharia de Telecomunicações e Controle II. $t$. 
Aos meus pais

Cleto de Sousa Caduda

e Irene Guimarães Caduda 
Este trabalho foi realizado com o apoio financeiro do Conselho Nacional do Desenvolvimento Científico e Tecnológico - CNPq

Agradeço também à EMBRAER pela oportunidade de analisar arrays utilizados em túnel de vento. 


\section{Agradecimentos}

Ao Prof. Dr. Luiz Antonio Baccalá pela orientação paciente, as muitas críticas construtivas, e o estímulo para a pesquisa.

Aos colegas Amanda de Paula, Lucas Massarope e Gilson Vieira pelas discussões e contribuições feitas no transcorrer deste trabalho.

A minha companheira Vânia Costa pela ajuda na revisão, e pelo apoio e compreensão.

A todos que de alguma forma contribuíram e involutariamente foram omitidos. 


\section{Resumo}

O estudo concentra-se na localização de fontes de ruídos em aeroacústica, através do processamento digital de sinais. O objetivo em aeroacústica é localizar fontes de ruído em estruturas aerodinâmicas (e.g.: aerofólios, slats, flaps e trens de pouso), motores e turbinas. Isto se faz possível utilizando arranjos de microfones, ou simplesmente arrays, cujos sinais são processados para localizar as fontes. Ao utilizar o beamforming clássico para processar os sinais vindos do array, este é incapaz de localizar as fontes de ruído de forma satisfatória. O 2D-ESPRIT é um método de alta resolução que é apresentado como alternativa. Nas simulações, é possível perceber que o 2D-ESPRIT tem melhor desempenho que o beamforming clássico, conseguindo localizar fontes próximas com arrays quadrados e com um número reduzido de amostras de sinal.

Palavras-chave: Array ESPRIT Aeroacústica Beamforming Acústica Imageamento Acústico 


\section{Abstract}

This study focuses on locating sources of noise in aeroacoustics, through digital signal processing. An objective in aeroacoustics is to locate sources of noise in aerodynamic structures (e.g.: airfoils, slats, flaps and landing gears), engines and fans. This is possible using microphone arrays, whose signals are processed to locate the sources. Using classical beamforming as the processing scheme for these signals, it is shown that it is incapable of locating sources satisfactorily in many of the practical scenarios. 2D-ESPRIT is a high resolution processing scheme that is presented as an alternative. Simulations show that 2D-ESPRIT outperfoms classical beamforming, locating closely positioned sources with the simple URA and with a reduced number of signal samples.

Keywords: Array ESPRIT Aeroacoustics Beamforming Acoustic Imaging 


\section{Sumário}

Resumo i

Abstract ii

Lista de Figuras vi

Lista de Símbolos vii

$\begin{array}{lll}1 & \text { Introdução } & 1\end{array}$

2 Conceitos Básicos 3

2.1 Arraus . . . . . . . . . . . . . . . . . . . . . . 3

2.2 Regiões de Operacão do Arrau . . . . . . . . . . . . . . . . . . . . 5

2.3 Fontes acústicas em campo distante . . . . . . . . . . . . . . . . 6

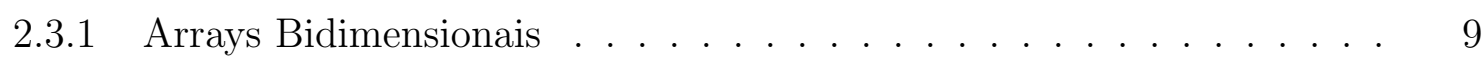

2.4 Fontes acústicas em campo próximo . . . . . . . . . . . . . . 10

\begin{tabular}{lll}
\hline & Processamento Digital de Sinais em Arrays & 14
\end{tabular}

3.1 Beamforming Clássico . . . . . . . . . . . . . . . . . . . . . 14

3.2 Estimacão Espectral Não-Paramétrica . . . . . . . . . . . . . . . . . . . . 17

3.3 Métodos de Deconvolução . . . . . . . . . . . . . . . . . . . . . . . 18

4 Alta Resolucão em Arrays 20

4.1 ESPRIT . . . . . . . . . . . . . . . . . . . . . . 20

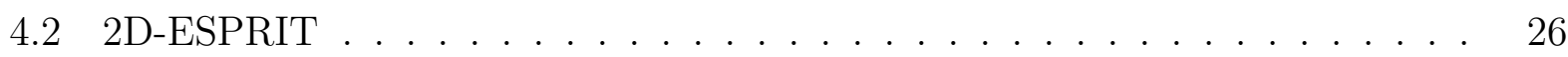

4.2 .1 O Problema do Pareamento . . . . . . . . . . . . . . 27

4.2 .2 2D-ESPRIT como Solução do Pareamento . . . . . . . . . . . 28

4.2 .3 Comentários Finais . . . . . . . . . . . . . . . . . . . 38

\begin{tabular}{ll|}
5 & Resultados \\
\hline
\end{tabular}

5.1 Beamforming Clássico . . . . . . . . . . . . . . . . . . . . . . . . 39

5.2 Alta Resolução aplicando 2D-ESPRIT . . . . . . . . . . . . . . . . . 41 
SUMÁRIO

6 Conclusões $\quad 46$

6.1 Contribuições . . . . . . . . . . . . . . . . . . . . . 47

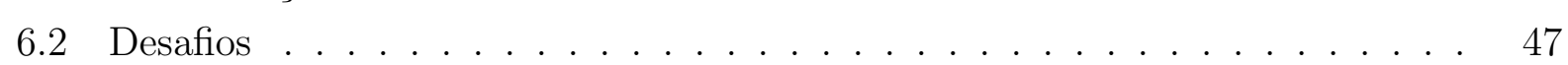

$\begin{array}{ll}\text { A Teoria da Difração } & 49\end{array}$

Referências Bibliográficas $\quad 52$ 


\section{Lista de Figuras}

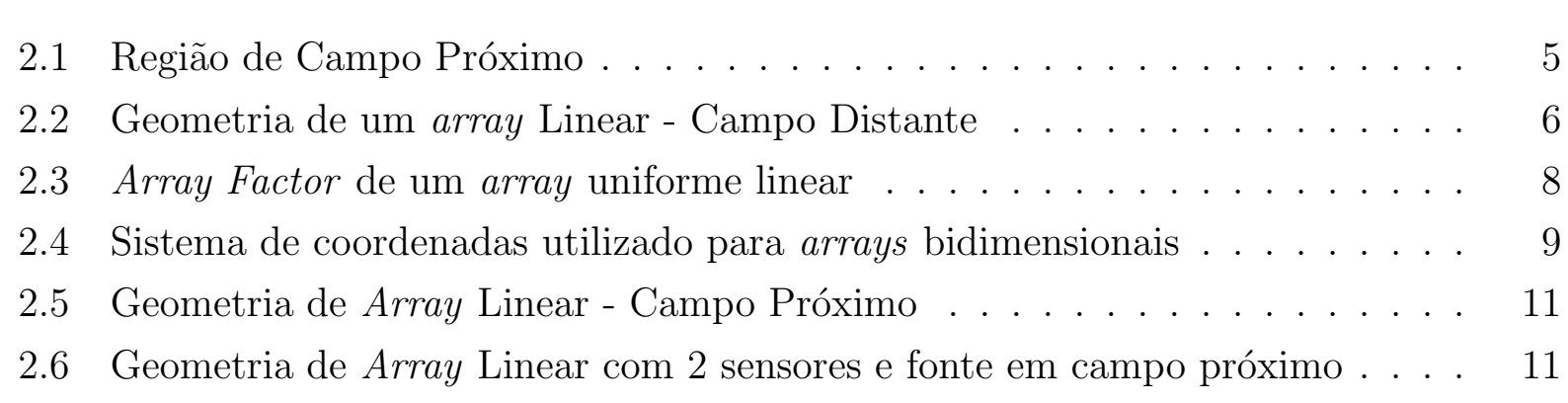

3.1 Diagrama de blocos para "Delay $\&$ Sum" . . . . . . . . . . . . . . . . 15

4.1 Configuracão típica para uso do ESPRIT . . . . . . . . . . . . . . . . . . . . 21

4.2 A diferenca de fase entre dois arraus $X$ e $Y \ldots \ldots . \ldots . \ldots . \ldots 22$

4.3 Aplicacão do ESPRIT em dois vetores de deslocamento . . . . . . . . . . . . 27

4.4 Exemplos de arraus com centro simetria utilizáveis no 2D-ESPRIT . . . . . 28

4.5 Array quadrado com 16 elementos dispostos uniformemente . . . . . . . . . 29

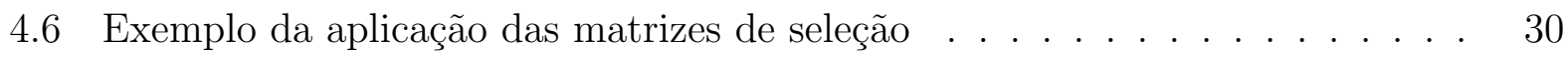

4.7 Par de sensores $1-4$ do array $2 \times 2$ em destaque . . . . . . . . . . . . . . . . 30

5.1 Array de 4 sensores com fonte pontual tonal - Beamforming Clássico . . . 40

5.2 Imageamento acústico para fonte em campo distante - Beamforming Clássico 40

5.3 Imageamento acústico para fonte em campo próximo - Beamforming Clássico 40

5.4 Geometria para 2 fontes - Beamforming Clássico . . . . . . . . . . . . 41

5.5 Imageamento de campo distante para 2 fontes tonais - Beamforming Clássico 41

5.6 Imageamento de campo próximo para 2 fontes tonais - Beamforming Clássico 41

5.7 Imagem acústica gerada por beamforming clássico para uma fonte tonal . . . 42

5.8 Imagem acústica gerada por 2D-ESPRIT para uma fonte tonal . . . . . . . . 42

5.9 Erros de localizacão para arrav quadrado $4 \times 4$. . . . . . . . . . . . . . 43

5.10 Imagem acústica gerada por beamforming clássico para 2 fontes tonais . . . 44

5.11 Imagem acústica gerada por 2D-ESPRIT para 2 fontes tonais . . . . . . . . 44

5.12 Imagem acústica gerada por 2D-ESPRIT com 4 fontes e 800 amostras . . . . 44

5.13 Imagem acústica gerada por 2D-ESPRIT com 4 fontes e 1000 amostras . . . 45

5.14 Imagem acústica gerada por beamforming clássico com 4 fontes . . . . . . . 45

5.15 Imagem acústica gerada por 2D-ESPRIT com 4 fontes . . . . . . . . . . . . 45 
6.1 Diagrama de uso dos módulos do software desenvolvidos . . . . . . . . . . 48

A.1 Condicão experimental para observar a difracão . . . . . . . . . . . . . . 50

A.2 Experimento de Young para estudo de padrões de interferência . . . . . . . . . 50 


\section{Lista de Símbolos}

$\gamma_{x}, \gamma_{z}$ Autovalores generalizados

$\lambda \quad$ Comprimento de onda

$\mathbf{a}(\theta, \phi)$ "Steering Vector"

$\mathbf{E}_{s} \quad$ Matriz de autovetores do sinal

J Matriz de seleção

k Vetor de onda

$\mathbf{P}_{s} \quad$ Matriz de potências

r Vetor de direção radial

$\mathbf{R}_{x x} \quad$ Matriz de covariância do array X

$\mathbf{R}_{x y} \quad$ Matriz de covariância cruzada entre os arrays $X$ e $Y$

$\mathbf{S}(f)$ Matriz de densidades espectrais de potência

$\omega \quad$ Frequência angular em rads/s

$\phi \quad$ Elevação

$\sigma^{2} \quad$ Potência do ruído branco aditivo

$\theta \quad$ Azimute

$\vec{\Delta} \quad$ Vetor de deslocamento entre arrays idênticos

c Velocidade do som em $\mathrm{m} / \mathrm{s}$

d Número de fontes a serem localizadas

E[.] Média temporal

$k \quad$ Número de onda 
M Número de sensores no array

$m_{x} \quad$ Número de sensores nos subarrays deslocados no eixo $X$

$m_{x} \quad$ Número de sensores nos subarrays deslocados no eixo $Z$

$p \quad$ Pressão sonora em $P a$

$P_{y} \quad$ Potência recebida no array

$R \quad$ Distância radial da fonte até um ponto qualquer

$S \quad$ Amplitude da pressão sonora na superfície de uma fonte sonora pontual

$s(t) \quad$ Sinal recebido no array

$t \quad$ Tempo em segundos

$w \quad$ Pesos para ajuste de fase de sinais

$y(t) \quad$ Sinal resultante da soma ponderada dos sinais recebidos no array 


\section{Capítulo 1}

\section{Introdução}

O presente trabalho estuda a aplicação de imageamento acústico para localizar e caracterizar fontes de sons e ruídos utilizando redes de microfones, doravante referidos simplesmente como arrays. Arrays deste tipo tem sido largamente estudados e tem particular interesse em acústica [3]. Suas primeiras aplicações remontam à Primeira Guerra Mundial (ca. 1917) [9] num contexto em que militares tinham interesse em medir a distância até artilharia e aviões inimigos. Desde então, a tecnologia evoluiu para soluções eletrônicas, de tamanho compacto e que não necessitam de movimento mecânico para direcionar o array. Atualmente, temos aplicações de arrays acústicos para videoconferência [11], [10], soluções viva-voz para telefonia [24], e prospecção em geologia através de imageamento sísmico [28].

Em aeroacústica, métodos computacionais (DNS - Direct Numerical Simulation para a solução das equações de Navier-Stokes, com ruído associado a fluxo turbulento [22], 23]) aplicados ao fluxo aerodinâmico são importantes para determinar fontes de ruído aeroacústico e para entender detalhes de seus mecanismos de geração. Ocorre, porém, que estes métodos numéricos requerem muito tempo de processamento sob elevados requisitos de poder computacional para que se possa reconhecer a área geradora de ruído. A aplicação de arrays para imageamento acústico tem em princípio o papel de informar a localização das fontes de ruído produzindo uma economia computacional uma vez que regiões de interesse podem ter discretização mais refinada somente nas regiões de interesse definidas pelo array. É neste sentido que o imageamento acústico gerado através de arrays serve para localizar e caracterizar fontes de ruído provenientes da estrutura de aeronaves, jatos e hélices [19], e acaba sendo uma ferramenta útil no estudo de mecanismos geradores de ruído aeroacústico.

A principal limitação de arrays em imageamento acústico diz respeito à sua resolução espacial que descreve a capacidade do array discriminar fontes sonoras adjacentes. A forma clássica de operar com arrays é através do chamado beamforming clássico, descrito na Sec. 3.1 cujo desempenho costuma ser inadequado quanto ao quesito resolução, sobretudo em frequências baixas. O procedimento usual para lidar com esta limitação é projetar arrays com geometrias complexas e que tenham maior diretividade.

A esta altura vale a pena observar que a teoria clássica de beamforming tem forte 
analogia com problemas de estimação espectral, pois ambas podem ser entendidas como representações na forma de transformadas de Fourier respectivamente a partir dos domínios do espaço e do tempo. Na realidade esta forma de interpretar o problema serve como meio para explicar as limitações do método clássico que representa uma espécie de dual espacial da estimação espectral não paramétrica [26].

É neste contexto que se insere o presente estudo que pretende enfocar o chamado método ESPRIT também estudado no contexto de estimação espectral e que por sua natureza paramétrica permite que se obtenha alta resolução.

Em aeroacústica, a implementação direta do ESPRIT tem a priori duas limitações: (a) sua versão espacial requer que os sinais sejam de banda estreita, e (b) a disposição dos sensores precisa ser uniforme e regular. A hipótese de banda estreita é facilmente superada através de uma decomposição do sinal em bandas e a segunda conflita diretamente com as geometrias não-uniformes geralmente empregadas em aeroacústica e portanto requer que se estudem adaptações. Parte do presente estudo é provar que há vantagem em adotar estruturas menos complexas do que as atualmente empregadas, e que as limitações são compensadas através do processamento feito no contexto do ESPRIT.

O presente texto se estrutura da seguinte forma: o funcionamento dos arrays é discutido conceitualmente na Sec. 2.1 segundo as hipóteses dos chamados (a) campo distante ou (b) campo próximo (Sec. 2.3 e 2.4 respectivamente). Indicamos que a formatação de feixe ou beamforming clássico tem desempenho inadequado na Sec. 3.1 e buscamos nos métodos de estimação espectral de alta-resolução uma forma mais eficiente de determinar as posições das fontes sonoras no Cap, 4. Foco é dado ao ESPRIT na Sec. 4.1 como um método interessante para esta aplicação e na Sec. 4.2 apresentamos a extensão do ESPRIT para arrays bidimensionais, o 2D-ESPRIT. No Cap. 5, comparamos o desempenho do 2D-ESPRIT com o beamforming clássico, apresentando resultados gerados a partir de simulações feitas em MatLab. Finalizamos com um apanhado do estudo no Cap. 6] e indicamos os próximos passos da aplicação do ESPRIT em arrays. 


\section{Capítulo 2}

\section{Conceitos Básicos}

Como qualquer ferramenta que é empregada em engenharia, há a necessidade de conhecê-la a fundo para que seja possível usá-la de forma ótima. Conhecer as deficiências e limitações permite visualizar as possíveis formas de melhorar a ferramenta, portanto, estuda-se primeiro o funcionamento de arrays. Com estes conceitos básicos de arrays, pode-se implementar os métodos de processamento de dados de forma consciente e crítica.

A propagação de ondas acústicas é brevemente revista com ênfase na recepção de multiplas fontes por um sensor, na Sec. 2.1. A operação do array em campo distante ou campo próximo descrito na Sec. 2.2, aponta equacionamentos diferentes apresentados nas Secs. 2.3 e 2.4. Ainda nas Secs. 2.3 e 2.4 são feitos os equacionamentos para arrays lineares e para arrays bidimensionais ou planares.

\section{$2.1 \quad$ Arrays}

Um array é definido como um conjunto de sensores distribuídos espacialmente, cuja posição é conhecida "a priori". É este conhecimento que torna possível a formatação eletrônica do seu feixe, direcionando-o para regiões específicas do espaço sem a necessidade de mover o array fisicamente. Isso na prática equivale a uma filtragem espacial que permite "focar" o array apenas nas regiões de interesse, simplesmente ponderando de forma adequada as saídas dos sensores. O procedimento é análogo ao feito em filtros de tempo discreto do tipo FIR (Finite Impulse Response) no domínio do tempo. Para escolher os termos multiplicativos deste filtro espacial é preciso entender o comportamento de um array.

A teoria básica de funcionamento da formatação de feixes no array provém da equação de onda, comumente utilizada no estudo de oscilações elétricas em condutores, vibrações mecânicas longitudinais e transversais, e vibrações em gases para citar alguns exemplos. A propagação acústica segue também este modelo [20]. Como um exemplo de aplicação da equação de onda em acústica, vamos considerar o caso unidimensional com uma fonte pontual harmônica. A equação de onda para pressão sonora neste caso é: 


$$
\frac{\partial^{2} p(x, t)}{\partial x^{2}}=\frac{1}{c^{2}} \frac{\partial^{2} p(x, t)}{\partial t^{2}}
$$

com $c$ a velocidade do som em $m / s, p$ a pressão sonora em $P a$, e $t$ o tempo em segundos.

Esta equação parcial diferencial tem soluções na forma:

$$
p(x, t)=A e^{j(\omega t-k x)}+B e^{j(\omega t+k x)}
$$

com $A$ e $B$ escolhidos para satisfazer condições de contorno específicas e em que, $\omega=2 \pi f$ a frequência angular e $k=\frac{2 \pi}{\lambda}$ o número de onda. Há então duas parcelas na solução que indicam a presença de ondas se propagando em direções opostas.

Em três dimensões, o resultado se generaliza utilizando as coordenadas cartesianas $(x, y, z)$. Estamos interessados apenas em uma das parcelas de Eq. (2.2) já que desejamos captar a onda em sensores. Para uma onda plana se propagando em uma única direção dada pelo vetor de onda $\mathbf{k}=\left(k_{x}, k_{y}, k_{z}\right)$ a pressão sonora é:

$$
p(x, y, z, t)=A e^{j\left(\omega t-k_{x} x-k_{y} y-k_{z} z\right)}
$$

Em notação vetorial, com vetores $\mathbf{k}=k_{x} \hat{\mathbf{x}}+k_{y} \hat{\mathbf{y}}+k_{z} \hat{\mathbf{z}}$ e $\mathbf{r}=x \hat{\mathbf{x}}+y \hat{\mathbf{y}}+z \hat{\mathbf{z}}$ onde $(\hat{\mathbf{x}}, \hat{\mathbf{y}}, \hat{\mathbf{z}})$ são versores do plano Cartesiano, a equação 2.3 fica:

$$
p(\mathbf{r}, t)=A e^{j(\omega t-\mathbf{k} \cdot \mathbf{r})}
$$

Esta solução para a equação de onda é utilizada no estudo da propagação esférica de uma fonte pontual. A onda esférica proveniente de uma fonte sonora pontual sofre uma atenuação proporcional a distância da fonte até um ponto qualquer [20], então $A=\frac{S}{R}$, com $S$ representando a pressão sonora na superfície da fonte pontual e $R$ a distância da fonte até um ponto qualquer. Com esta consideração, a pressão sonora fica sendo:

$$
p(\mathbf{r}, t)=\frac{S}{R} e^{j(\omega t-\mathbf{k} \cdot \mathbf{r})}
$$

Com a eq. (2.5), calcula-se a propagação da pressão sonora de uma fonte pontual até um ponto $(x, y, z)$ qualquer do espaço. Aplicando o princípio da reciprocidade acústica [20], as posições de fonte e de sensor são intercambiáveis. Assim, a eq. (2.5) é usada para determinar a pressão sonora ao redor de um sensor ao invés de uma fonte.

Em geral, definem-se arrays com $N$ sensores, para cálculo da propagação acústica. A superposição da propagação acústica de cada elemento é a resposta acústica do array.

$$
p_{\text {array }}(\mathbf{r}, t)=\frac{1}{N} \sum_{i=1}^{N} w_{i}(t) p_{i}(\mathbf{r}, t)
$$

Incluem-se os pesos $w_{i}(t)$ para ajustar a fase dos sinais captados em cada sensor, formatando o feixe do array. Veremos mais detalhes sobre a aplicação destes pesos na Sec. 3.1 . 


\subsection{Regiões de Operação do Array}

Para simplificar o tratamento dos dados coletados pelo array, é útil identificar a região de operação do array. Até este momento, considerou-se a propagação esférica em torno de uma fonte pontual, mas a medida que nos distanciamos da fonte, a propagação se torna praticamente plana. A distância na qual podemos fazer esta aproximação é determinada pela teoria da difração usualmente estudada em óptica [17]. O efeito é característico do modelo de propagação por ondas e acontece quando parte da frente de onda encontra um obstáculo. Difrações são classificadas como de Fraunhofer ou de Fresnel (de campo distante ou campo próximo respectivamente) [1, [17]. O apêndice \ apresenta uma revisão de óptica física e do efeito de difrações.

Para definir as regiões de campo distante e de campo próximo, faz-se aproximações da equação de difração de Fresnel-Kirchhoff [4]. A equação é uma representação matemática do princípio de Huygens-Fresnel. Para uma dedução mais detalhada ver [4], 13] e [15].

A região de campo distante é dada por:

$$
r_{\min }>\frac{D^{2}}{4 \lambda}
$$

em [20]. Alguns autores preferem ser mais conservadores definindo fronteiras mais afastadas do array para garantir que seu funcionamento seja de campo distante. Como exemplo citamos [1] que define a região de campo distante como $R>\frac{2 D^{2}}{\lambda}$, ou 8 vezes maior do que aquela apresentada em (2.7). Por ser uma aproximação, deve-se escolher aquela que é mais adequada e portanto mantemos a definição dada pela Eq. (2.7).

A Eq. (2.7) permite definir uma região esférica em torno do array que representa a transição entre um campo e o outro, como mostra a Fig. 2.1:

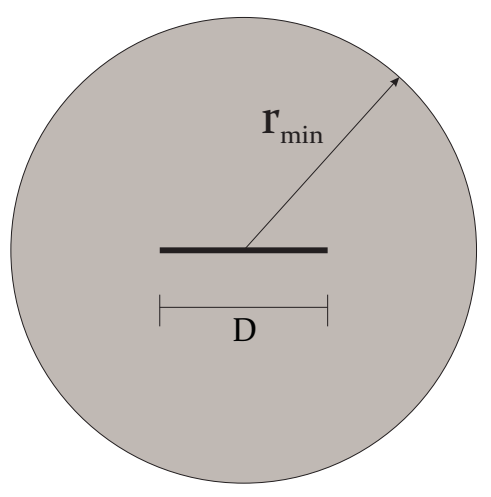

Figura 2.1: Região de Campo Próximo definida pela esfera de raio $r_{\min }$ e array cuja maior dimensão é D.

O funcionamento do array em campo distante permite determinar a direção de fontes em relação ao centro do array enquanto que em campo próximo pode-se determinar também a distância do array até a fonte. No campo próximo, é importante identificar esta distância 
do array até a fonte para obter uma imagem acústica com nitidez. Este efeito é análogo a imagens captadas por câmeras fotográficas onde o foco é ajustado para tirar um retrato. Para cada campo é feita uma abordagem diferente nos cálculos da propagação de onda. As Secs. 2.3 e 2.4, descrevem os conceitos envolvidos em cada campo e como abordá-los quantitativamente.

Aplicações de imageamento acústico em aeroacústica utilizam tanto um quanto o outro campo. Em medidas de túnel de vento o array opera em campo próximo, dado o espaço relativamente pequeno entre o modelo e a parede do túnel onde se posiciona o array. Quando o teste é feito com uma aeronave que sobrevoa o solo acima do array no chamado ensaio fly-over [25], o array opera em campo distante.

\subsection{Fontes acústicas em campo distante}

O funcionamento em campo distante é demonstrado através de um exemplo unidimensional. Neste exemplo tem-se um array linear de $N$ elementos, uniformemente espaçados sobre o eixo $x$. A Fig. 2.2 ilustra este array.

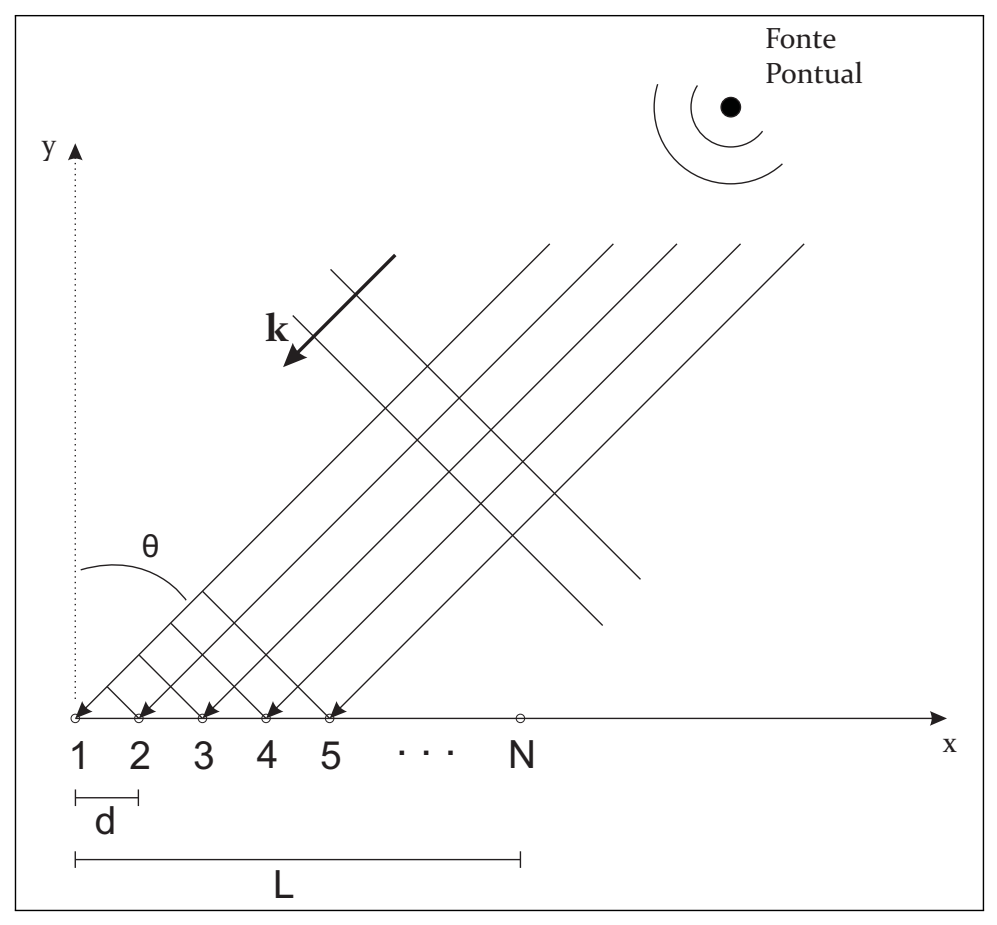

Figura 2.2: Geometria de um array Linear de N sensores uniformemente espaçados e fonte em campo distante com onda $\vec{k}$ atingindo o eixo com ângulo $\theta$.

Uma fonte pontual tonal está localizada a uma distância $R$ medida a partir do primeiro sensor $n=1$, chamado de sensor de referência. $R$ é muito maior que o comprimento do array $L(R \gg L)$, tal que a condição em (2.7) é atendida. Nesta situação, a propagação da onda sonora é praticamente plana ao chegar no array e a recepção nos sensores é proveniente 
sempre de uma mesma direção. Os ângulos são medidos a partir do eixo y. Para cada sensor a pressão sonora é:

$$
p_{n}\left(\mathbf{r}_{n}, t\right)=\frac{S}{\left|\mathbf{r}_{n}\right|} e^{j\left(\omega t-\mathbf{k} \cdot \mathbf{r}_{n}\right)}
$$

para $n=1,2, \ldots, N$ sensores do array. Definindo os vetores $\mathbf{k}=k\left(\sin \left(\theta_{k}\right) \mathbf{x}+\cos \left(\theta_{k}\right) \mathbf{y}\right)$ e $\mathbf{r}=R(\sin (\theta) \mathbf{x}+\cos (\theta) \mathbf{y})$, a pressão sonora no sensor de referência $n=1$ é:

$$
p_{1}\left(\mathbf{r}_{1}, t\right)=\frac{S}{R} e^{j\left(\omega t-k R \cos \left(\theta_{k}-\theta\right)\right)}
$$

Nos demais sensores do array, os vetores $\left\{\mathbf{r}_{n}, n=2,3,4, \ldots, N\right\}$ têm seus módulos sucessivamente decrementados por $d \sin (\theta)$ :

$$
\begin{array}{cc}
\left|r_{2}\right|= & (R-d \sin (\theta)) \\
\left|r_{3}\right|= & (R-2 d \sin (\theta)) \\
\vdots \\
\left|r_{N}\right|= & (R-(N-1) d \sin (\theta))
\end{array}
$$

e a pressão sonora para os $N$ sensores é,

$$
\begin{aligned}
& p_{2}\left(\mathbf{r}_{2}, t\right)=\frac{S}{\left|\mathbf{r}_{2}\right|} e^{j\left(\omega t-(k R-k d \sin (\theta)) \cos \left(\theta_{k}-\theta\right)\right)} \\
& p_{3}\left(\mathbf{r}_{3}, t\right)=\frac{S}{\left|\mathbf{r}_{3}\right|} e^{j\left(\omega t-(k R-2 k d \sin (\theta)) \cos \left(\theta_{k}-\theta\right)\right)} \\
& p_{N}\left(\mathbf{r}_{N}, t\right)=\frac{S}{\left|\mathbf{r}_{N}\right|} e^{j\left(\omega t-(k R-(N-1) k d \sin (\theta)) \cos \left(\theta_{k}-\theta\right)\right)}
\end{aligned}
$$

A atenuação devida ao termo $\frac{1}{\left|\mathbf{r}_{n}\right|}$ é aproximada para $\frac{1}{R}$ já que $R \gg L \quad$ e $L>d \Rightarrow$ $R \gg d$. Assim, para calcular a resposta do array, a soma de todos os elementos é:

$$
\begin{aligned}
p(\theta, t) & =\frac{1}{N} \sum_{n=1}^{N} \frac{S}{R} e^{j\left(\omega t-k R \cos \left(\theta_{k}-\theta\right)+(n-1) k d \sin (\theta) \cos \left(\theta_{k}-\theta\right)\right)} \\
& =\frac{S}{N R} e^{j\left(\omega t-k R \cos \left(\theta_{k}-\theta\right)\right)} \sum_{n=1}^{N} e^{j\left((n-1) k d \sin (\theta) \cos \left(\theta_{k}-\theta\right)\right)}
\end{aligned}
$$

A somatória

$$
A F(\theta)=\frac{1}{N} \sum_{n=1}^{N} e^{j\left((n-1) k d \sin (\theta) \cos \left(\theta_{k}-\theta\right)\right)}
$$


presente na Eq. (2.9) é chamada de fator do array (Array Factor) ou PSF (Point Spread Function) e depende somente das posições dos sensores e da direção descrita pelo ângulo $\theta$, dada uma fonte pontual. Como $|A F(\theta)| \leq 1$, o fator do array indica o ganho em uma determinada direção. A Fig. 2.3 ilustra o comportamento do fator do array para diferentes direçoes de um array uniforme linear com $N=5$ sensores, comprimento de onda $\lambda=0.343 \mathrm{~m}$, espaçamento entre sensores $d=\frac{\lambda}{2}$ e $\theta_{k}=0$ (fonte localizada a frente do array no eixo $y$ ). Pela Fig. 2.3, fica claro o comportamento de um filtro espacial. O array prioriza sinais vindos de fontes na direção $\theta=0$ aplicando ganho igual a 1 , e atenuando os sinais vindos de outras direções. Algumas direções apresentam nulos na Fig. 2.3. Fontes presentes nestas direções são efetivamente ignorados pelo array. Idealmente deseja-se que este filtro seja máximo para uma dada direção $\theta$, e nulo para todas as outras direções. Isto não é possível devido ao array ter tamanho limitado e ser constituído por um número discreto de sensores. Estas características estão relacionadas com a geração de lóbulos laterais. Na prática, isto implica que fontes acústicas localizadas em outras direções contribuem para a potência do sinal recebido pelo array, prejudicando a medida precisa da potência da fonte acústica na direção desejada do array. No projeto do arrays, procura-se minimizar o pico dos lóbulos laterais ajustando a quantidade e a posição dos sensores.

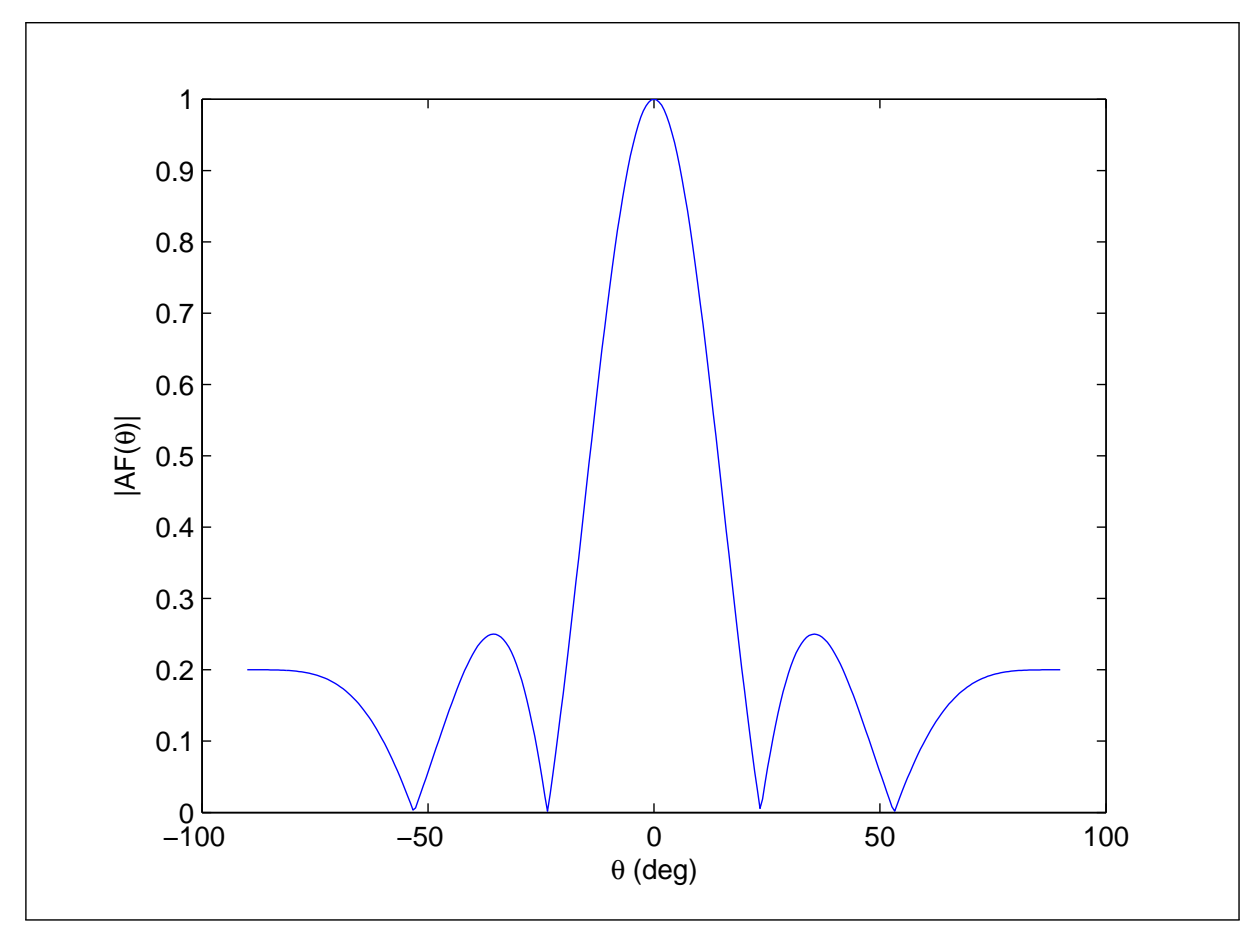

Figura 2.3: Array Factor de um array uniforme linear com $N=5$ sensores, espaçamento entre sensores $d=\frac{\lambda}{2}$ e $\operatorname{com} \theta_{k}=0$

A resposta do array naturalmente privilegia fontes acústicas localizadas a frente no eixo $y$, ou seja, para o ângulo $\theta=0$. É possível, utilizando pesos multiplicativos nas saídas dos sinais vindos dos sensores, modificar esta resposta que por sua vez modifica o ganho para direções que não sejam a central, $\theta \neq 0$. A direção é variada modificando o termo $k d \sin (\theta)$ 
da exponencial, para $k d \sin (\theta+\alpha)$. $\mathrm{O} \alpha$, em radianos, modifica a resposta do array para uma direção desejada. Pode-se então variar $\alpha$ para pontos escolhidos previamente com o objetivo de varrer o semiplano, medindo a intensidade da pressão acústica. Esta varredura eletrônica é vista em detalhes na Sec. 3.1.

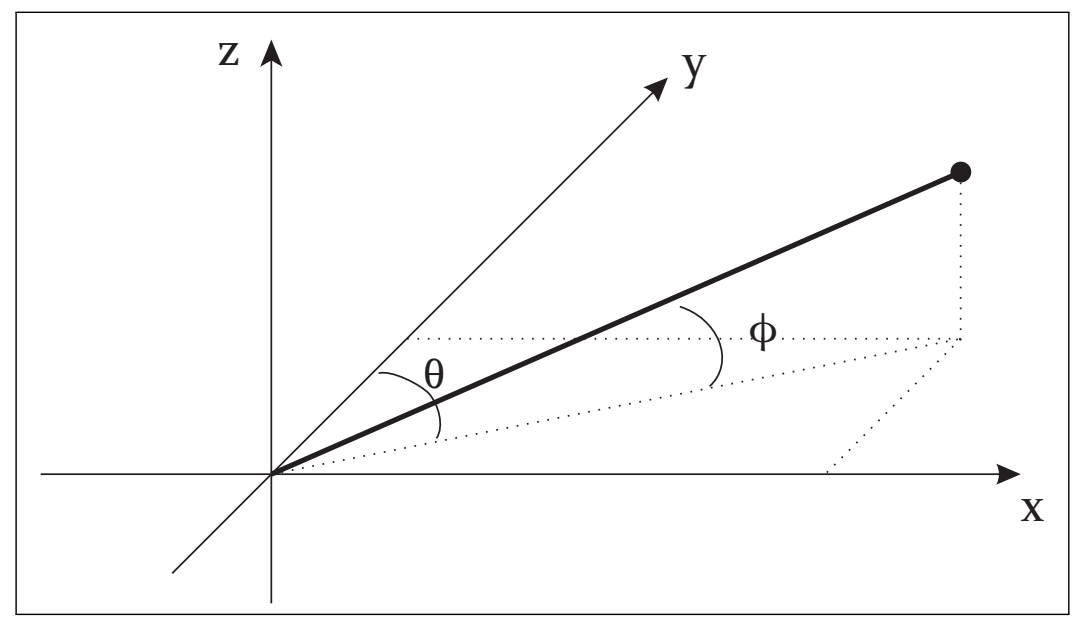

Figura 2.4: Sistema de coordenadas utilizado para arrays bidimensionais

\subsubsection{Arrays Bidimensionais}

Arrays unidimensionais limitam-se a varrer o plano $X Y$. Em aplicações de imageamento acústico são utilizados arrays bidimensionais, para varrer um espaço tridimensional. A resposta do array bidimensional é obtida estendendo os conceitos vistos anteriormente no final de Sec. 2.3 para a configuração de sensores posicionados sobre um mesmo plano. O sistema de coordenadas utilizado está ilustrado na Fig. 2.4. A posição do sensor $n=1$ coincide com a origem das coordenadas e os $N-1$ outros sensores são posicionados sobre o plano $X Z$. A pressão sonora para $n=1$ é:

$$
p_{1}\left(\mathbf{r}_{1}, t\right)=\frac{S}{R} e^{j\left(\omega t-k R\left(\cos ^{2}\left(\frac{\theta_{k}-\theta}{2}\right) \cos \left(\phi_{k}-\phi\right)+\sin ^{2}\left(\frac{\theta_{k}-\theta}{2}\right) \cos \left(\phi_{k}+\phi\right)\right)\right)}
$$

Cada um dos outros sensores terá uma distância $\left|\mathbf{r}_{n}\right|$ até a fonte que, para esta configuração, é re-escrita em função de $R$ utilizando coordenadas esféricas:

$$
\left|\mathbf{r}_{n}\right|=R-\left(x_{n} \sin (\theta) \sin (\phi)+z_{n} \cos (\theta) \sin (\phi)\right)
$$

em que, as posições dos sensores no plano são dadas pelas coordenadas $\left.\left(x_{n}, 0, z_{n}\right)\right)$. Os vetores $\mathbf{k}$ e $\mathbf{r}$ são expressos como:

$$
\begin{aligned}
\mathbf{k} & =|\mathbf{k}|\left(\cos \left(\phi_{k}\right) \hat{\mathbf{x}}+\sin \left(\phi_{k}\right) \cos \left(\theta_{k}\right) \hat{\mathbf{y}}+\sin \left(\phi_{k}\right) \sin \left(\theta_{k}\right) \hat{\mathbf{z}}\right) \\
\mathbf{r}_{n} & =\left|\mathbf{r}_{n}\right|(\cos (\phi) \hat{\mathbf{x}}+\sin (\phi) \cos (\theta) \hat{\mathbf{y}}+\sin (\phi) \sin (\theta) \hat{\mathbf{z}})
\end{aligned}
$$


e o produto escalar $\mathbf{k} \cdot \mathbf{r}_{n}$ é:

$$
\mathbf{k} \cdot \mathbf{r}_{n}=k\left|\mathbf{r}_{n}\right| \underbrace{\left(\cos ^{2}\left(\frac{\theta_{k}-\theta}{2}\right) \cos \left(\phi_{k}-\phi\right)+\sin ^{2}\left(\frac{\theta_{k}-\theta}{2}\right) \cos \left(\phi_{k}+\phi\right)\right)}_{=\beta_{k}}
$$

As pressões sonoras medidas nestes sensores são dadas por:

$$
\begin{gathered}
p_{2}\left(\mathbf{r}_{2}, t\right)=\frac{S}{\left|\mathbf{r}_{2}\right|} e^{j\left(\omega t-k\left(R-x_{2} \sin (\theta) \sin (\phi)-z_{2} \cos (\theta) \sin (\phi)\right) \beta_{k}\right)} \\
p_{3}\left(\mathbf{r}_{3}, t\right)=\frac{S}{\left|\mathbf{r}_{3}\right|} e^{j\left(\omega t-k\left(R-x_{3} \sin (\theta) \sin (\phi)-z_{3} \cos (\theta) \sin (\phi)\right) \beta_{k}\right)} \\
\vdots \\
p_{N}\left(\mathbf{r}_{N}, t\right)=\frac{S}{\left|\mathbf{r}_{N}\right|} e^{j\left(\omega t-k\left(R-x_{N} \sin (\theta) \sin (\phi)-z_{N} \cos (\theta) \sin (\phi)\right) \beta_{k}\right)}
\end{gathered}
$$

E finalmente a resposta do array bidimensional é:

$$
p(\theta, \phi, t)=\frac{S}{N R} e^{j\left(\omega t-k R \beta_{k}(\theta, \phi)\right)} \sum_{n=1}^{N} e^{j k\left(x_{n} \sin (\theta) \sin (\phi)+z_{n} \cos (\theta) \sin (\phi)\right) \beta_{k}(\theta, \phi)}
$$

A resposta do array bidimensional depende das coordenadas (em $\theta$ e $\phi$ ) e o filtro espacial atua em ambas coordenadas. Igualmente ao que foi feito com arrays lineares, o lóbulo principal deste array é direcionado para $(\theta, \phi)$ escolhidos para varrer o espaço tridimensional.

\subsection{Fontes acústicas em campo próximo}

No campo próximo, a distância do array até a fonte é pequena e a onda que chega nos elementos não é plana. Para ilustrar, considere o array linear de $N$ elementos da Fig. 2.5, com uma fonte pontual tonal.

Com a propagação esférica, não há maneira simples para relacionar os atrasos de chegada da frente de onda nos sensores como foi no caso de fonte no campo distante. Um exemplo simples aplicando a Eq. (2.5) é um array de $N=2$ sensores apresentado na Fig. 2.6 .

A equação da pressão sonora para cada elemento é:

$$
\begin{aligned}
& p_{1}(\mathbf{r}, t)=\frac{S}{\left|\mathbf{r}-\mathbf{r}_{1}\right|} e^{j\left(w t-\mathbf{k} \cdot\left(\mathbf{r}-\mathbf{r}_{1}\right)\right)} \\
& p_{2}(\mathbf{r}, t)=\frac{S}{\left|\mathbf{r}-\mathbf{r}_{2}\right|} e^{j\left(w t-\mathbf{k} \cdot\left(\mathbf{r}-\mathbf{r}_{2}\right)\right)}
\end{aligned}
$$

O produto escalar nas Eqs. (2.14) e (2.15) dependem dos ângulos $\theta_{1}$ e $\theta_{2}$, ilustrados na Fig. 2.6. Pode-se relacionar estes ângulos com o ângulo $\theta$ formado pelo vetor de posição $\mathbf{r}$, tal 


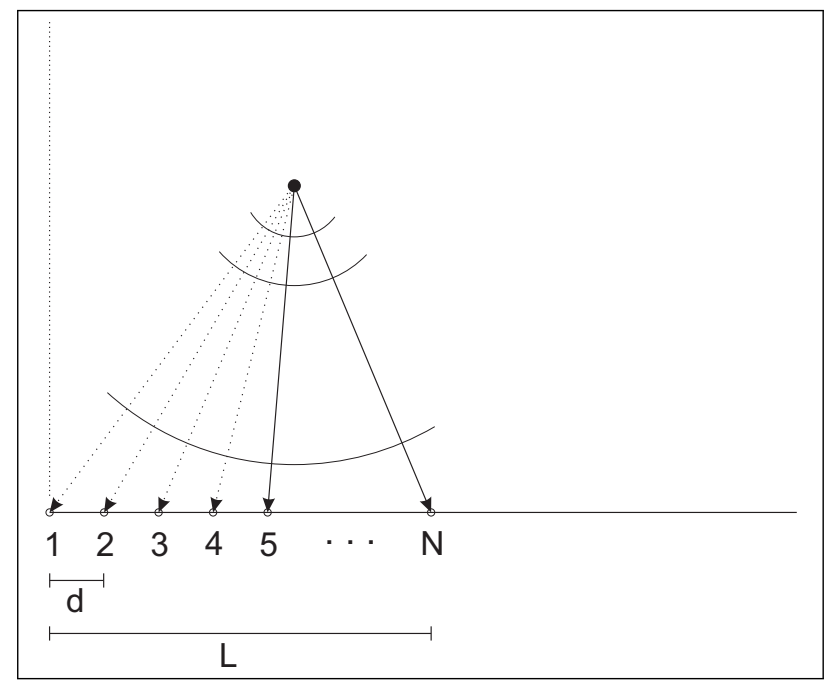

Figura 2.5: Geometria de Array Linear de $N$ elementos uniformemente espaçados no eixo $x$. Propagação de onda com fonte em campo próximo.

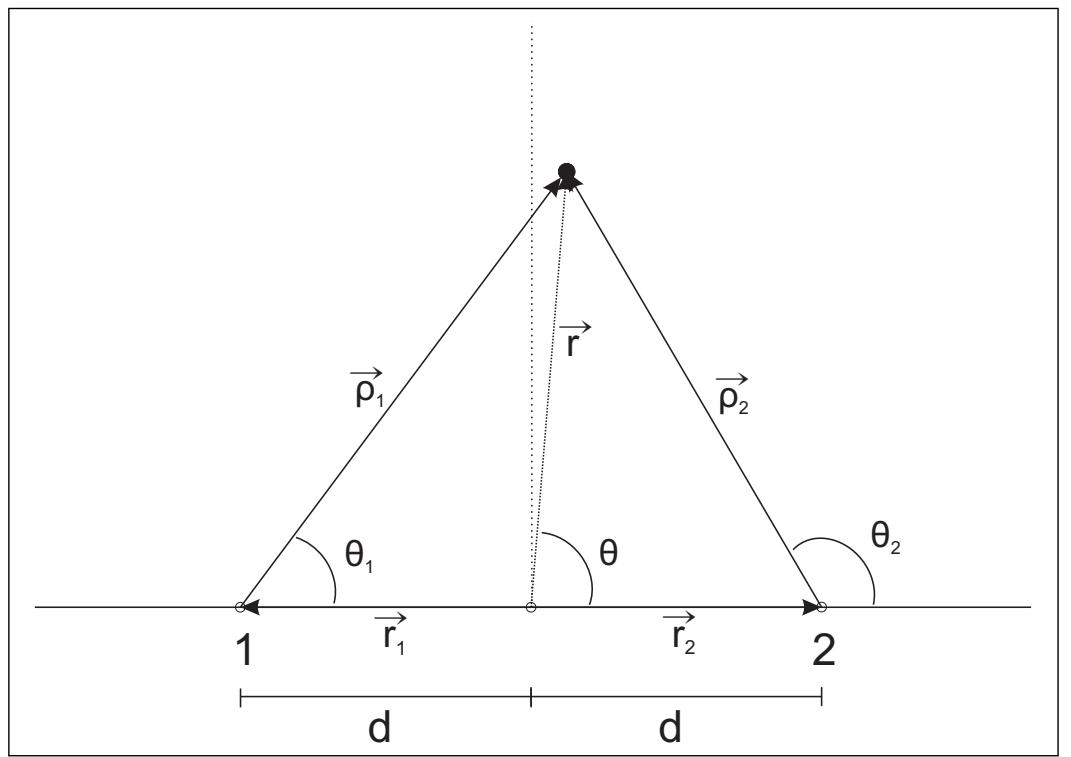

Figura 2.6: Geometria de Array Linear com 2 sensores e fonte em campo próximo

que:

$$
\begin{aligned}
& \theta_{1}=\arccos \left(\frac{|\mathbf{r}| \cos (\theta)-\left|\mathbf{r}_{1}\right|}{\left|\mathbf{r}-\mathbf{r}_{1}\right|}\right) \\
& \theta_{2}=\arccos \left(\frac{|\mathbf{r}| \cos (\theta)-\left|\mathbf{r}_{2}\right|}{\left|\mathbf{r}-\mathbf{r}_{2}\right|}\right)
\end{aligned}
$$

Considerando a geometria do array os módulos são substituídos por: 


$$
\begin{aligned}
& \left|\mathbf{r}-\mathbf{r}_{1}\right|=\sqrt{r^{2}+d^{2}-2 r d \cos (\theta)} \\
& \left|\mathbf{r}-\mathbf{r}_{2}\right|=\sqrt{r^{2}+d^{2}+2 r d \cos (\theta)}
\end{aligned}
$$

e portanto, os ângulos são:

$$
\begin{aligned}
& \theta_{1}=\arccos \left(\frac{r \cos (\theta)+d}{\sqrt{r^{2}+d^{2}-2 r d \cos (\theta)}}\right) \\
& \theta_{2}=\arccos \left(\frac{r \cos (\theta)-d}{\sqrt{r^{2}+d^{2}+2 r d \cos (\theta)}}\right)
\end{aligned}
$$

Com estas relações, a resposta do array é:

$$
p(\mathbf{r}, t)=\frac{S e^{j \omega t}}{2}\left(\frac{e^{-j k\left|\mathbf{r}-\mathbf{r}_{1}\right| \cos \left(\theta_{k}-\theta_{1}\right)}}{\left|\mathbf{r}-\mathbf{r}_{1}\right|}+\frac{e^{-j k\left|\mathbf{r}-\mathbf{r}_{2}\right| \cos \left(\theta_{k}-\theta_{2}\right)}}{\left|\mathbf{r}-\mathbf{r}_{2}\right|}\right)
$$

Fazendo a substituição dos módulos pelas Eqs. (2.19) e (2.20):

$$
p(r, \theta, t)=\frac{S e^{j w t}}{2}\left(\frac{e^{-j k \beta_{k, 1} \sqrt{r^{2}+d^{2}-2 r d \cos (\theta)}}}{\sqrt{r^{2}+d^{2}-2 r d \cos (\theta)}}+\frac{e^{-j k \beta_{k, 2} \sqrt{r^{2}+d^{2}+2 r d \cos (\theta)}}}{\sqrt{r^{2}+d^{2}+2 r d \cos (\theta)}}\right)
$$

Os $\beta_{k, 1}$ são definidos como sendo:

$$
\begin{aligned}
& \beta_{k, 1}(r, \theta)=\cos \left(\theta_{k}-\theta_{1}\right) \\
& \beta_{k, 2}(r, \theta)=\cos \left(\theta_{k}-\theta_{2}\right)
\end{aligned}
$$

E para um número $N$ qualquer de sensores, a resposta do array é:

$$
p(r, \theta, t)=\frac{S e^{j w t}}{N} \sum_{n=1}^{N} \frac{e^{-j k \beta_{k, n} \sqrt{r^{2}+x_{n}^{2}+2 r x_{n} \cos (\theta)}}}{\sqrt{r^{2}+x_{n}^{2}+2 r x_{n} \cos (\theta)}}
$$

em que $x_{n}$ é a posição do sensor na reta $X$.

Pela Eq. (2.27), existe dependência na direção (ângulo $\theta$ ) e na distância (r). Para configurações em campo próximo temos mais informação a respeito da localização da fonte.

Assim como feito para o campo distante, os conceitos de campo próximo são extendidos para arrays bidimensionais (situados no plano XZ) com $N$ sensores, escrevendo a norma do vetor $\boldsymbol{\rho}_{n}$ como sendo: 


$$
\begin{aligned}
\left|\boldsymbol{\rho}_{n}\right| & =\left|\mathbf{r}-\mathbf{r}_{n}\right| \\
& =\sqrt{|\mathbf{r}|^{2}+\left|\mathbf{r}_{n}\right|^{2}-2 \mathbf{r} \cdot \mathbf{r}_{n}} \\
& =\sqrt{r^{2}+\left(x_{n}^{2}+z_{n}^{2}\right)-2 r \cos (\phi)\left(x_{n} \sin (\theta)+z_{n} \cos (\theta)\right)}
\end{aligned}
$$

em que $r$ é a distância do sensor de referência até a fonte, $x_{n}$ e $z_{n}$ são as coordenadas no plano XZ dos $n$ sensores, e $\phi, \theta$ os ângulos do vetor de direção do array. Substituindo a Eq. (2.28) na resposta do array temos:

$$
p(r, \theta, \phi, t)=\frac{S e^{j \omega t}}{N} \sum_{n=1}^{N} \frac{e^{-j k \beta_{k, n} \sqrt{r^{2}+\left(x_{n}^{2}+z_{n}^{2}\right)-2 r \cos (\phi)\left(x_{n} \sin (\theta)+z_{n} \cos (\theta)\right)}}}{\sqrt{r^{2}+\left(x_{n}^{2}+z_{n}^{2}\right)-2 r \cos (\phi)\left(x_{n} \sin (\theta)+z_{n} \cos (\theta)\right)}}
$$

Em resumo, a combinação dos sensores no array formam uma resposta direcionada e esta funciona como um filtro espacial. É importante especificar qual é o campo no qual se situa a fonte para poder analisar corretamente os dados coletados pelo array. Na Sec. 3.1, aplicam-se pesos nas saídas de cada elemento permitem direcionar a resposta do array para uma direção desejada e com um processamento de sinais adequado é possível construir uma imagem acústica. Em todas as respostas do array nota-se a dependência do número de onda $|\mathbf{k}|=\frac{2 \pi}{\lambda}$, e portanto, o array tem resposta diferente para cada comprimento de onda (ou frequência) diferente. Esta característica não é vista como um problema quando consideramos sinais de fontes de banda estreita, já que a resposta espacial para a dada frequência de interesse é conhecida e tem variação desprezível. No entanto, para fontes de banda larga, como em acústica, temos que varrer uma faixa de frequências e para cada uma, o filtro espacial varia. Para construir a imagem acústica é necessário ter a resposta do array para toda a faixa de interesse. Para evitar que em algumas frequências a resolução de fontes seja melhor do que em outras, soluções como a CDB (Constant Directivity Beamforming) [5] procuram manter a resposta espacial constante no projeto e construção do array, para uma larga faixa de frequências. 


\section{Capítulo 3}

\section{Processamento Digital de Sinais em Arrays}

As Secs. 3.1 a 3.3 a seguir, mostram como extrair a posição das fontes acústicas através dos dados coletados nos sensores do array e utilizando os conceitos explicados na Sec. 2.1. O método clássico é realizar a varredura do espaço mudando adequadamente os pesos aplicados na saída dos sensores [33]. Este método tem uma limitação na sua resolução espacial que dificulta ou até mesmo impossibilita a identificação de duas fontes pontuais distintas. Em especial quando incluímos ruído branco presente nas medidas dos sensores, a localização das fontes pela simples inspeção da imagem acústica pode ficar inviável.

Existem métodos que procuram melhorar a imagem acústica através de processos iterativos. São os chamados algoritmos de deconvolução utilizados originalmente em rádio astronomia. Dentre estes métodos destacamos na Sec. 3.3 o CLEAN[18] e DAMAS[6]. Os algoritmos de deconvolução são bons desde que as estimativas iniciais das posições das fontes também sejam boas. Além dos algoritmos de deconvolução existem os métodos de alta-resolução que permitem soluções com melhor desempenho e maior precisão na estimativa da posição das fontes. Em particular o ESPRIT, cuja descrição se encontra no Cap. 4, e que comparado ao beamforming clássico, apresenta uma solução vantajosa. Adicionalmente o ESPRIT serve como estimativa inicial para os algoritmos de deconvolução, garantindo convergências nas iterações presentes nestes métodos.

\subsection{Beamforming Clássico}

Nas Secs. 2.3 e 2.4, o uso de pesos multiplicativos adequados nos sensores do array permite direcioná-lo, alterando sua resposta espacial. O beamforming clássico, o Delay $\mathscr{G}$ Sum, ou atraso e soma, aplica estes pesos, ou neste caso simplesmente pesos atrasadores, diretamente na saída dos sensores do array. Os atrasadores são ajustados para apontar o feixe do array para uma direção desejada. A escolha dos atrasos, depende da posição dos 
sensores e a direção do feixe que se deseja. A Fig. 3.1 ilustra o diagrama de blocos para o beamforming utilizando Delay \& Sum.

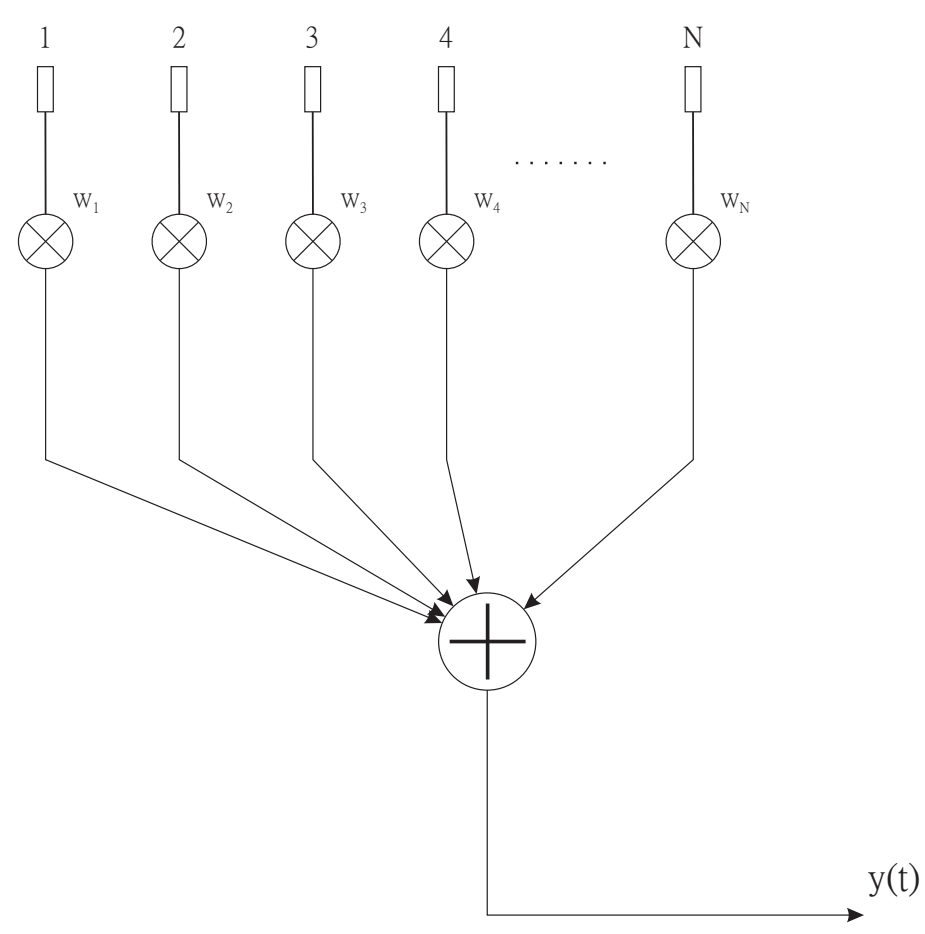

Figura 3.1: Diagrama de blocos para "Delay ES Sum"

Considera-se a seguir que o array recebe um sinal de banda estreita, ou seja, todos os parâmetros são para uma frequência $f_{0}$. Lembramos que em acústica o sinal recebido é de banda larga, e portanto, é necessário varrer as frequências (ou comprimentos de onda) dentro da faixa de interesse. Os pesos multiplicativos na saída de cada um dos elementos são definidos como:

$$
w_{i}(\theta, \phi, r)=e^{-j \psi_{i}(\theta, \phi, r)}
$$

Os pesos servem para aplicar uma defasagem nos sinais dos sensores, formatando o feixe do array. O sinal na saída do somador $y(t)$ é a combinaçao das saídas de cada sensor ponderados por $w_{i}(\theta, \phi, r)$ :

$$
y(t)=\sum_{i=1}^{N} w_{i}(\theta, \phi, r) s_{i}(t)
$$

Aqui vemos a semelhança com filtragem temporal do tipo FIR. Mas, diferente da filtragem temporal, os valores amostrados nos $N$ sensores para um mesmo instante $t$ são a entrada do filtro, ou seja, é realizada uma amostragem espacial e consequentemente há uma filtragem espacial. Nesta filtragem, direções distintas são filtradas basta escolher os valores $\omega_{i}(\theta, \phi, r)$ adequados que posicionam o feixe nas direções desejadas. A Eq. 3.2 na forma vetorial é: 


$$
y(t)=\mathbf{s}^{T} \mathbf{w}
$$

onde,

$$
\mathbf{s}=\left[\begin{array}{c}
s_{1}(t) \\
s_{2}(t) \\
s_{3}(t) \\
\vdots \\
s_{N}(t)
\end{array}\right]
$$

é o vetor de sinais recebidos pelos $N$ sensores e

$$
\mathbf{w}=\left[\begin{array}{c}
w_{1}(\theta, \phi, r) \\
w_{2}(\theta, \phi, r) \\
w_{3}(\theta, \phi, r) \\
\vdots \\
w_{N}(\theta, \phi, r)
\end{array}\right]
$$

é o vetor de pesos aplicado antes do somador do array.

A imagem acústica é essencialmente a potência recebida de cada direção. O cálculo da potência proveniente das diversas direções $(\theta, \phi, r)$ é feito com o sinal $y(t)$ resultante do somador. Calcula-se a potência da seguinte maneira:

$$
\begin{aligned}
E\left[|y(t)|^{2}\right] & =E\left[y^{*}(t) y(t)\right] \\
& =E\left[\mathbf{w}^{H} \mathbf{s}^{*} \mathbf{s}^{T} \mathbf{w}\right] \\
& =\mathbf{w}^{H} \underbrace{E\left[\mathbf{s}^{*} \mathbf{s}^{T}\right]}_{=\mathbf{P}_{s}} \mathbf{w} \\
P_{y}(\theta, \phi, r) & =E\left[|y(t)|^{2}\right]=\mathbf{w}^{H} \mathbf{P}_{s} \mathbf{w}
\end{aligned}
$$

em que o termo $E\left[\mathbf{s}^{*}(t) \mathbf{s}^{T}(t)\right]=\mathbf{P}_{s}$ é a matriz de covariância espacial do array no instante $t$. Observa-se que a combinação dos sinais y tem dependência em $(\theta, \phi, r)$ e que por abuso de notação escreve-se apenas como $y(t)$. Como visto na Sec. 2.4, há a dependência de $r$ em $P_{y}$, que tem o efeito de focalização da fonte acústica.

Em geral, uma matriz de correlação é dada por $E\left[\mathbf{s}^{*}(t) \mathbf{s}^{T}(t+\tau)\right]$ e a sua transformada de Fourier equivale a:

$$
\mathcal{F}\left[E\left[\mathbf{s}^{*}(t) \mathbf{s}^{T}(t+\tau)\right]\right]=\mathbf{S}(f)
$$


em que os elementos da matriz são densidades espectrais de potência do sinal recebido no sensor na diagonal principal e o restante são densidades espectrais de potência cruzada dos sinais. No entanto, para a Eq. (3.4) tem-se que

$$
\left.\mathcal{F}\left[E\left[\mathbf{s}^{*}(t) \mathbf{s}^{T}(t+\tau)\right]\right]\right|_{\tau=0}
$$

Isto resulta em integrar as densidades espectrais de potência, os elementos da matriz $\mathbf{S}(f)$ para $\{f:-\infty<f<+\infty\}$. O resultado é a matriz $\mathbf{P}_{s}$ de potências que é utilizada de fato no cálculo da imagem acústica na Eq. (3.4).

Este é o contexto teórico para o cálculo da matriz $\mathbf{S}(f)$. No entanto, na prática, há um número limitado de amostras para calcular a matriz e é necessário recorrer a estimativas para os elementos da matriz $\mathbf{S}(f)$, calculando assim $\hat{\mathbf{S}}(f)$.

\subsection{Estimação Espectral Não-Paramétrica}

Devido à limitação do número de amostras e quantidade de realizações possíveis estimase a matriz de covariância $\mathbf{S}(f)$ por $\hat{\mathbf{S}}(f)$. Para este fim, o estimador mais intuitivo para as densidades espectrais de potência e densidades espectrais de potência cruzada (os elementos da matriz $\mathbf{S}(f)$ ) é o periodograma (3.7), usado em estimação espectral não-paramétrica [26], 30]. Para dois sinais $x(t)$ e $y(t)$ vindos de 2 sensores distintos do array as suas transformadas são $X(f)$ e $Y(f)$ respectivamente. Os peridogramas para as densidades espectrais de potência e densidades espectrais de potência cruzada são dados por:

$$
\hat{S}_{x}(f)=\frac{1}{N}|\hat{X}(f)|^{2}, \quad \hat{S}_{x y}(f)=\frac{1}{N} \hat{X}(f) \hat{Y}^{*}(f)
$$

A densidade espectral de potência (ou densidade espectral de potência cruzada) estimada através do periodograma tem limitações quanto a sua utilidade. Quando estima-se a densidade espectral de potência (ou o espectro cruzado), é desejável que:

1. $E\left[\hat{S}_{i j}(f)\right] \approx S_{i j}(f)$ para todo $f \mathrm{e}$,

2. $V A R\left[\hat{S}_{i j}(f)\right] \rightarrow 0$ para um número de amostras $N \rightarrow \infty$,

características denominadas de viés e consistência respectivamente. Demonstra-se em [26] que a primeira condição é valida apenas para alguns valores de frequência, enquanto que a segunda condição não é atendida. Para corrigir estas deficiências é necessário aplicar uma janela de dados para que a condição (1) seja atendida e uma janela da autocorrelação para a variância na condição (2) (ver [26]).

A janela de dados aplica pesos diferentes à cada uma das $N$ amostras de um processo estocástico estacionário antes do cálculo das transformadas de Fourier. Seu objetivo é reduzir o vazamento de potência espectral. A estimativa da densidade espectral de potência com o janelamento de dados é obtida por: 


$$
\hat{S}^{\prime}(f)=\left|\sum_{k=1}^{N} h(k) s(k) e^{-j 2 \pi f k}\right|^{2}
$$

A janela é representada por uma sequência $h(k)$ de comprimento $N$, cujos termos tendem a zero suavemente em suas extremidades, em $h(1)$ e $h(N)$. Exemplos de janelas utilizadas são Hanning, Hamming, Blackman e Kaiser, sendo que a mais utilizada é a janela de Hamming.

A janela de autocorrelação $w(\tau)$ de tamanho $2 N-1$ e sua transformada de Fourier $W(f)$ tem como objetivo suavizar o espectro. O estimador utilizando esta janela é calculado aplicando a convolução da janela transformada $W(f)$ sobre o espectro estimado com a janela de dados de (3.8):

$$
\hat{S}^{w}(f)=\int_{-f(N)}^{f(N)} W(f-\alpha) \hat{S}^{\prime}(\alpha) d \alpha
$$

com o qual resulta um espectro com menos variância. Um exemplo é a chamada janela de Bartlett:

$$
w(\tau)= \begin{cases}1-\frac{|\tau|}{m}, & |\tau|<m \\ 0, & |\tau| \geq m\end{cases}
$$

em que $m \leq N$. Bartlett (1950) argumentou que a Eq. (3.10) poderia reduzir as flutuações devidas a amostragem do peridograma dividindo a sequência original de tamanho $N$ em $N / m$ blocos com $m$ amostras cada. Os periodogramas dos $m$ blocos são calculados e a média destes periodogramas é o resultado final. Na realidade, este procedimento de medianização dos periodogramas equivale a aplicar (3.10) em (3.9). Welch(1967) melhorou esta técnica, argumentando que ao dividir os blocos com sobreposição (50\% das amostras do bloco anterior são incluídas no próximo bloco) é possível reduzir a variância além da obtida pelo método de Bartlett [26]. Outras janelas utilizadas são as janelas de Parzen, Daniell e Papoulis.

Estes métodos reduzem as características indesejáveis do periodograma e são aplicados na estimativa dos elementos da matriz em (3.5). No entanto, mesmo com estes esforços há restrições na resolução de fontes como se vê nos resultados de simulações nas Figs. 5.5 e 5.6 no Cap. 5, em que o reconhecimento de 2 fontes pontuais não é trivial. Em aplicações práticas, o efeito é que a imagem formada pode esconder informações sobre outras fontes presentes. Nos métodos de alta-resolução é encontrada uma solução para este problema.

\subsection{Métodos de Deconvolução}

Uma forma de melhorar os resultados obtidos pelo beamforming clássico é através dos algoritmos de deconvolução. Levando em consideração que o array é um sistema óptico, a imagem acústica obtida é o resultado da convolução da resposta unitária do array, ou seja a PSF, e as fontes acústicas. Os algoritmos de deconvolução visam realizar o processo 
inverso. A PSF do array é conhecida, dada a sua geometria, e teoricamente pode-se retirar as distorções e imperfeições que o array ocasiona na imagem acústica através da deconvolução. No entanto, a inversão não é bem condicionada devido a presença de nulos na PSF. Para contornar este problema, os algoritmos de deconvolução são iterativos. A cada passada identificam uma fonte na imagem acústica orignalmente gerada pelo beamforming clássico. O algoritmo pára por um critério que deve ser escolhido de forma a não confundir fontes com o ruído. Os resultados são bons apesar da elevada carga computacional [12].

O algoritmo CLEAN 18] para deconvolução foi desenvolvido para aplicações de radio astronomia de alta resolução. O algoritmo começa com a imagem acústica 'suja', obtida pelo beamforming clássico, e a PSF. A cada iteração busca-se na imagem 'suja' o maior pico e subtrai-se a PSF centrada neste ponto. Isto se repete até que o maior pico da imagem resultante das sucessivas subtrações está no nível do ruído. Neste momento interrompemos a iteração e o algoritmo termina remontando uma imagem 'limpa' através das subtrações feitas nos passos anteriores.

Apesar de funcionar em diversos casos, o CLEAN apresenta problemas quando há a presença de fontes correlacionadas. Há também a seleção do critério de interrupção do algoritmo que escolhe-se para que seja o valor médio do ruído presente na medida. No entanto, se houver fontes próximas deste valor médio, ou se for impossível medir este valor médio, pode-se perder fontes que estavam presentes através do algoritmo.

A dificuldades do CLEAN em ambientes em que ocorrem reflexões (túneis de vento de seção fechada), foi uma motivação para o desenvolvimento do algoritmo DAMAS [6] especificamente para aplicações aeroacústicas. O algoritmo é mais adequado a medidas aeroacústicas oferecendo imagens melhores do que o CLEAN ao considerar a reverberação que ocorre em túneis de vento. O artigo [6] mostra que são necessários mais de 1000 iterações para o algoritmo convergir obtendo imagens satisfatórias. 


\section{Capítulo 4}

\section{Alta Resolução em Arrays}

Algoritmos de alta resolução para determinar a direção de chegada (DoA - Direction of Arrival) e para estimação espectral são comuns em processamento digital de sinais, como por exemplo, os algoritmos CAPON [7], MUSIC [29] e Root-MUSIC [2]. O MUSIC foi o primeiro a mostrar os benefícios da decomposição em autovalores da matriz de covariância, determinando o DoA através do espaço de sinal construído com os autovetores da decomposição. De interesse particular é o ESPRIT (Estimation of Signal Parameters by Rotational Invariance Technique) [27] que como o MUSIC utiliza a decomposição em autovalores da matriz de correlação, mas evita a varredura dos ângulos de chegada. O ESPRIT tem fácil implementação e produz estimativas precisas com relativamente poucas amostras. Estas vantagens fazem o ESPRIT um candidato interessante para a aplicação em aeroacústica, pois o beamforming clássico tem processamento longo devido a varredura, produzindo resultados pouco confiáveis, e para conseguir boas resoluções com grandes faixas dinâmicas as geometrias dos arrays tornam-se complexas, onerando o processamento. Ao aplicá-lo em aeroacústica foi necessário estender o ESPRIT para arrays planares usualmente empregados. Como o array planar permite determinar 2 parâmetros da direção (azimute e elevação com relação ao centro do array), a dificuldade estaria em parear os valores para cada fonte de ruído. O 2D-ESPRIT [16] apresenta uma extensão que permite parear corretamente os valores de azimute e elevação, e compartilha com o ESPRIT uma implementação simples. Nas Secs. 4.1 e 4.2 que seguem, detalhamos o ESPRIT e sua extensão, o 2D-ESPRIT.

\subsection{ESPRIT}

O ESPRIT consegue através do deslocamento de arrays idênticos, localizar fontes com alta resolução. Assim como seres humanos localizam fontes sonoras através da diferença de fase sentida entre os ouvidos, o ESPRIT se utiliza da diferença de fase causada pelo deslocamento entre dois arrays idênticos.

Considere os arrays idênticos $X$ e $Y$, deslocados por um vetor $\vec{\Delta}$, como ilustra a Fig. 4.1. Ambos arrays recebem ondas planas de fontes de banda estreita localizadas no campo 


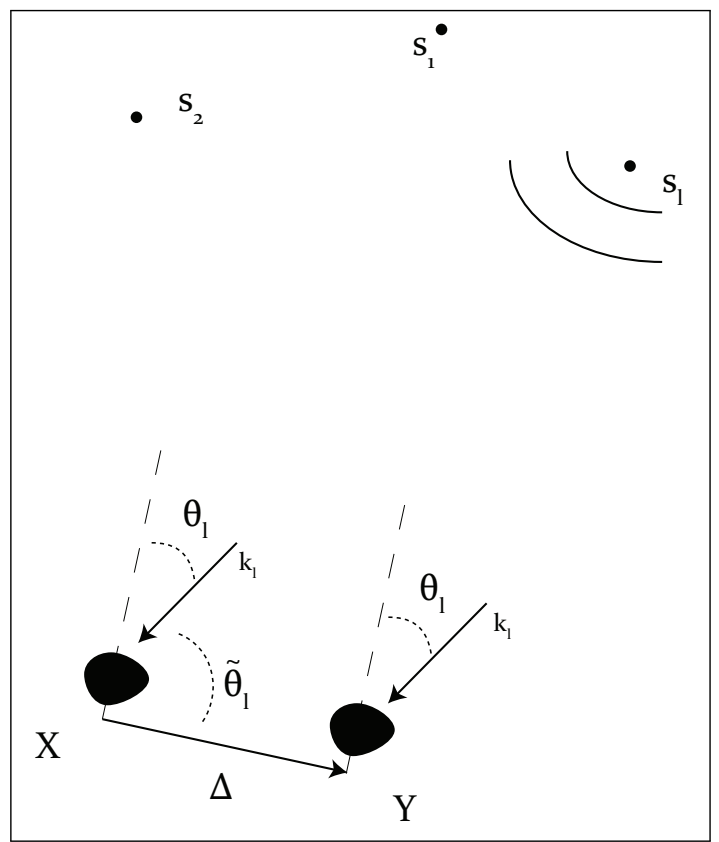

Figura 4.1: Configuração típica para uso do ESPRIT. Cada sinal $s_{k}$ das fontes localizadas em campo distante é recebida pelos arrays $X$ e $Y$. Deseja-se determinar o ângulo $\theta_{l}$ que é a direção da onda plana incidente nos arrays referente ao vetor de deslocamento $\vec{\Delta}$.

distante. O problema se resume em localizar as $d$ fontes presentes através dos ângulos $\theta_{l}$, com $l=1,2, \cdots, d$ que são os complementares dos ângulos $\widetilde{\theta}_{l}$ formados entre o vetores de propagação de onda $\vec{k}_{l}$ e o vetor de deslocamento $\vec{\Delta}$. Para determinar os ângulos $\theta_{l}$, calculam-se os produtos internos:

$$
\overrightarrow{k_{l}} \cdot \vec{\Delta}=\left|\vec{k}_{l}\right||\vec{\Delta}| \cos \left(\widetilde{\theta}_{l}\right)
$$

e fazendo a substituição $\widetilde{\theta}_{l}=\frac{\pi}{2}-\theta_{l}$ na Eq. (4.1), resulta em:

$$
\begin{aligned}
\vec{k}_{l} \cdot \vec{\Delta} & =\left|\vec{k}_{l}\right||\vec{\Delta}| \cos \left(\frac{\pi}{2}-\theta_{l}\right) \\
& =\left|\vec{k}_{l}\right||\vec{\Delta}|\left(\cos \left(\frac{\pi}{2}\right) \cos \left(\theta_{l}\right)+\sin \left(\frac{\pi}{2}\right) \sin \left(\theta_{l}\right)\right) \\
& =\left|\vec{k}_{l}\right||\vec{\Delta}| \sin \left(\theta_{l}\right)
\end{aligned}
$$

O resultado é exatamente a diferença de fase entre a onda que atinge o array $X$ e $Y$, já que, devido ao deslocamento, a onda plana atinge um array antes do outro. A diferença de fase é dependente da diferença entre a distância da fonte até o array $X$ e a distância da fonte até o array $Y$ é dado por:

$$
\zeta=|\vec{\Delta}| \sin \left(\theta_{l}\right)
$$

A Fig. 4.2 ilustra esta diferença. 


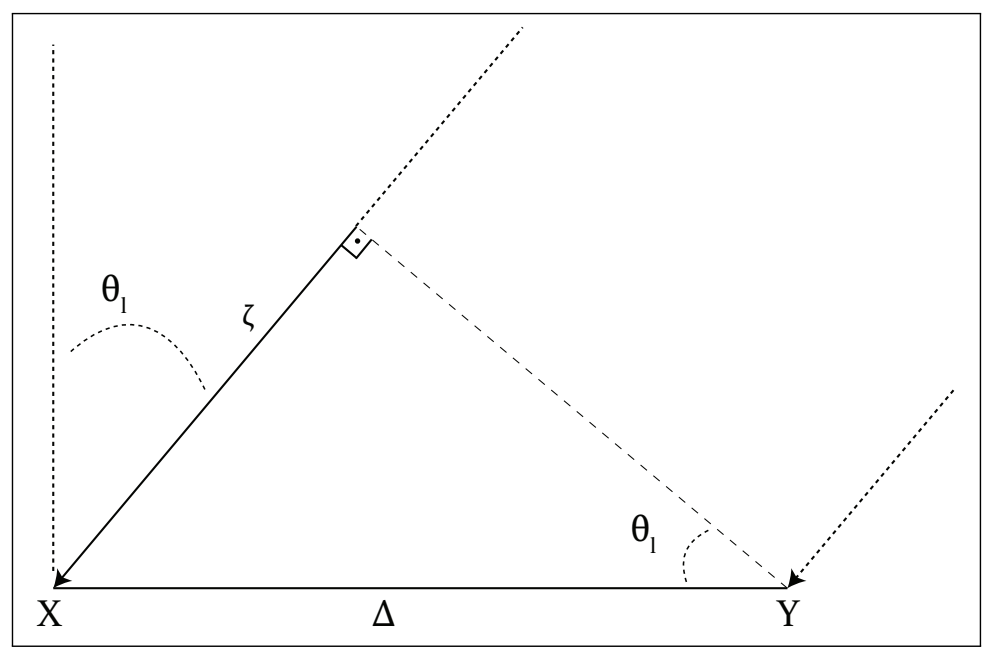

Figura 4.2: A diferença de fase entre dois arrays $X$ e $Y$. A diferença na distância percorrida pela onda plana para $X$ e para $Y$ depende do ângulo $\theta_{l}$.

Os sinais de banda estreita, definidos como:

$$
s_{l}(t)=A_{l} e^{j 2 \pi f t+\nu_{l}}
$$

com $A_{l}$ amplitude do sinal, $f$ a frequência do sinal e $\nu_{l}$ uma fase qualquer, permitem que um atraso temporal $\tau$ no sinal seja representado por:

$$
s_{l}(t-\tau)=s_{l}(t) e^{-j 2 \pi f \tau}
$$

Ao expressar atrasos como na Eq. (4.5), os sinais recebidos em cada sensor $i$ nos arrays $X$ e $Y$ são:

$$
\begin{aligned}
& x_{i}(t)=\sum_{l=1}^{d} s_{l}(t) a_{i}\left(\theta_{l}\right)+n_{x i}(t), \\
& y_{i}(t)=\sum_{l=1}^{d} s_{l}(t) a_{i}\left(\theta_{l}\right) e^{j k|\vec{\Delta}| \sin \left(\theta_{l}\right)}+n_{y i}(t), \quad(i=1,2, \cdots, m)
\end{aligned}
$$

em que, $n_{x i}(t)$ e $n_{y i}(t)$ são ruídos brancos aditivos de média nula e variância $\sigma^{2}$. $a_{i}\left(\theta_{l}\right)$ é o atraso do sinal no i-ésimo sensor relativo ao primeiro sensor do mesmo array, ou seja:

$$
a_{i}\left(\theta_{l}\right)=e^{j 2 \pi f \bar{\tau}_{i}\left(\theta_{l}\right)}
$$

Os $a_{i}$ 's estão diretamente relacionados a geometria do array, e ver-se-á que no ESPRIT é desnecessário conhecer estes parâmetros para determinar os ângulos $\theta_{l}$. O vetor formado pelos $a_{i}\left(\theta_{l}\right), i=1,2,3, \cdots, m$ é chamado de "steering vector". Cada ângulo $\theta_{l}$ tem seu "steering vector" associado, determinando os atrasos relativos aplicados a cada sensor do array para uma direção. 
A principal diferença entre as Eqs. (4.6) e (4.7) é a presença do fator $e^{j k|\vec{\Delta}| \sin \left(\theta_{l}\right)}$ que é a diferença de fase vista na Eq. (4.2) e relaciona os sinais dos arrays $X$ e $Y$. Esta ligação entre os dois arrays é que permite localizar as fontes.

Introduz-se a notação matricial para as Eqs. (4.6) e (4.7), combinando as saídas de cada sensor no seu respectivo array:

$$
\begin{aligned}
& \mathbf{x}(t)=\mathbf{A} \mathbf{s}(t)+\mathbf{n}_{x}(t) \\
& \mathbf{y}(t)=\mathbf{A} \boldsymbol{\Phi} \mathbf{s}(t)+\mathbf{n}_{y}(t)
\end{aligned}
$$

em que:

$$
\begin{aligned}
& \mathbf{x}=\left[\begin{array}{c}
x_{1}(t) \\
x_{2}(t) \\
x_{3}(t) \\
\vdots \\
x_{m}(t)
\end{array}\right] \\
& \mathbf{y}=\left[\begin{array}{c}
y_{1}(t) \\
y_{2}(t) \\
y_{3}(t) \\
\vdots \\
y_{m}(t)
\end{array}\right] \text {, } \\
& \mathbf{s}(t)=\left[\begin{array}{c}
s_{1}(t) \\
s_{2}(t) \\
s_{3}(t) \\
\vdots \\
s_{d}(t)
\end{array}\right] \\
& \mathbf{A}=\left[\begin{array}{ccccc}
a_{1}\left(\theta_{1}\right) & a_{1}\left(\theta_{2}\right) & a_{1}\left(\theta_{3}\right) & \ldots & a_{1}\left(\theta_{d}\right) \\
a_{2}\left(\theta_{1}\right) & a_{2}\left(\theta_{2}\right) & a_{2}\left(\theta_{3}\right) & \ldots & a_{2}\left(\theta_{d}\right) \\
a_{3}\left(\theta_{1}\right) & a_{3}\left(\theta_{2}\right) & a_{3}\left(\theta_{3}\right) & \ldots & a_{3}\left(\theta_{d}\right) \\
\vdots & \vdots & \vdots & \ddots & \vdots \\
a_{m}\left(\theta_{1}\right) & a_{m}\left(\theta_{2}\right) & a_{m}\left(\theta_{3}\right) & \ldots & a_{m}\left(\theta_{d}\right)
\end{array}\right]
\end{aligned}
$$

$\mathrm{e}$

$$
\boldsymbol{\Phi}=\operatorname{diag}\left(e^{j k|\vec{\Delta}| \sin \left(\theta_{l}\right)}\right), \quad l=1,2, \cdots, d
$$

A diferença de fase entre os sinais que chegam nos arrays $X$ e $Y$, é representada por $\boldsymbol{\Phi}$. Cada elemento da diagonal corresponde a uma fonte com seu respectivo $\theta_{l}$. Portanto, ao determinar a matriz $\boldsymbol{\Phi}$ as fontes são localizadas. A matriz $\boldsymbol{\Phi}$ é obtida comparando os sinais 
recebidos pelos arrays através da correlação e a correlação cruzada:

$$
\begin{aligned}
& \mathbf{R}_{x x}=E\left[\mathbf{x}(t) \mathbf{x}^{H}(t)\right] \\
& \mathbf{R}_{x y}=E\left[\mathbf{x}(t) \mathbf{y}^{H}(t)\right]
\end{aligned}
$$

em que, $\mathbf{x}^{H}(t)$ e $\mathbf{y}^{H}(t)$ são os conjugados transpostos de $\mathbf{x}(t)$ e $\mathbf{y}(t)$ respectivamente.

Utilizando as Eqs. (4.9) e (4.10) nas Eqs. (4.16) e (4.17):

$$
\begin{aligned}
\mathbf{R}_{x x} & =E\left[\mathbf{x}(t) \mathbf{x}^{H}(t)\right] \\
& =E\left[\mathbf{A} \mathbf{s}(t) \mathbf{s}^{H}(t) \mathbf{A}^{H}+\mathbf{A} \mathbf{s}(t) \mathbf{n}_{x}^{H}(t)+\mathbf{n}_{x}(t) \mathbf{s}^{H}(t) \mathbf{A}^{H}+\mathbf{n}_{x}(t) \mathbf{n}_{x}^{H}(t)\right] \\
& =\mathbf{A} E\left[\mathbf{s}(t) \mathbf{s}^{H}(t)\right] \mathbf{A}^{H}+E\left[\mathbf{n}_{x}(t) \mathbf{n}_{x}^{H}(t)\right] \\
& =\mathbf{A} \mathbf{R}_{s} \mathbf{A}^{H}+\sigma^{2} \mathbf{I} \\
\mathbf{R}_{x y} & =E\left[\mathbf{x}(t) \mathbf{y}^{H}(t)\right] \\
& =E\left[\mathbf{A} \mathbf{s}(t) \mathbf{s}^{H}(t) \mathbf{\Phi}^{H} \mathbf{A}^{H}+\mathbf{A} \mathbf{s}(t) \mathbf{n}_{y}^{H}(t)+\mathbf{n}_{x}(t) \mathbf{s}^{H}(t) \mathbf{\Phi}^{*} \mathbf{A}^{H}+\mathbf{n}_{x}(t) \mathbf{n}_{y}^{H}(t)\right] \\
& =\mathbf{A} E\left[\mathbf{s}(t) \mathbf{s}^{H}(t)\right] \mathbf{\Phi}^{H} \mathbf{A}^{H} \\
& =\mathbf{A} \mathbf{R}_{s} \mathbf{\Phi}^{H} \mathbf{A}^{H}
\end{aligned}
$$

Assumindo que as potências dos sinais sejam maiores que a potência do ruído, os menores autovalores de $\mathbf{R}_{x x}$ serão iguais a $\sigma^{2}$ e cada autovalor maior do que $\sigma^{2}$ é associado a potência de uma fonte [30]. Retira-se o rúido em $\mathbf{R}_{x x}$ definindo a matriz $\mathbf{C}$ :

$$
\mathbf{C}=\mathbf{R}_{x x}-\lambda_{\min } \mathbf{I}=\mathbf{A} \mathbf{R}_{s} \mathbf{A}^{H}
$$

Por simplicidade de notação define-se a matriz $\mathbf{D}$ :

$$
\mathbf{D}=\mathbf{R}_{x y}=\mathbf{A R}_{s} \Phi^{H} \mathbf{A}^{H}
$$

As matrizes $\mathbf{C}$ e $\mathbf{D}$ são similares exceto pela matriz $\boldsymbol{\Phi}$ que deseja-se determinar. A proposta é comparar as matrizes definindo a função matricial:

$$
\widetilde{\mathbf{X}}(\gamma)=\mathbf{C}-\gamma \mathbf{D}
$$

com $\gamma \in \mathbb{C}$ sendo o parâmetro livre. Deseja-se encontrar $\gamma^{\prime}$ s para que $|\widetilde{\mathbf{X}}(\gamma)|=0$, em que $\mid$.| é o determinante. Desta forma, os $\gamma$ 's que permitem fazer isto são os fatores que representam a diferença entre as duas matrizes, ou seja, representam de alguma forma a matriz $\mathbf{\Phi}$.

Após algumas manipulações algébricas na Eq. (4.22) obtém-se a diferença por:

$$
\widetilde{\mathbf{X}}(\gamma)=\mathbf{C}-\gamma \mathbf{D}=\mathbf{A} \mathbf{R}_{s} \mathbf{A}^{H}-\gamma \mathbf{A} \mathbf{R}_{s} \Phi^{H} \mathbf{A}^{H}=\mathbf{A R}_{s}\left(\mathbf{I}-\gamma \boldsymbol{\Phi}^{H}\right) \mathbf{A}^{H}
$$


Tomando o determinante em $\widetilde{\mathbf{X}}(\gamma)$ e igualando a zero:

$$
\begin{aligned}
|\widetilde{\mathbf{X}}(\gamma)| & =0 \\
\left|\mathbf{A R}_{s}\left(\mathbf{I}-\gamma \boldsymbol{\Phi}^{H}\right) \mathbf{A}^{H}\right| & =0
\end{aligned}
$$

nota-se que a Eq. (4.24) é igual a zero se a matriz $\mathbf{R}_{s}$ ou a matriz $\left(\mathbf{I}-\gamma \boldsymbol{\Phi}^{H}\right)$ é singular, já que as matrizes $\mathbf{A}$ e $\mathbf{A}^{H}$ apenas aplicam uma transformação linear do sinal para o espaço do array. A matriz $\mathbf{R}_{s}$ não é singular devido a sua construção que produz uma matriz de posto completo. Resta a matriz $\left(\mathbf{I}-\gamma \boldsymbol{\Phi}^{H}\right)$ que ao escolher $\gamma^{\prime}$ 's convenientes pode vir a ser singular. A matriz $\boldsymbol{\Phi}^{H}$ é:

$$
\boldsymbol{\Phi}^{H}=\operatorname{diag}\left(e^{-j k|\vec{\Delta}| \sin \left(\theta_{l}\right)}\right), \quad l=1,2, \cdots, d
$$

Para que $\left|\left(\mathbf{I}-\gamma \boldsymbol{\Phi}^{H}\right)\right|=0$, e consquentemente também $|\widetilde{\mathbf{X}}(\gamma)|=0$, apenas $\gamma_{l}=e^{j k|\vec{\Delta}| \sin \left(\theta_{l}\right)}, \quad l=$ $1,2, \cdots, d$ são soluções, e são exatamente os elementos da diagonal na matriz $\boldsymbol{\Phi}$ a determinar.

Numericamente os $\gamma$ 's são determinados através da decomposição em autovalores generalizados. Autovalores generalizados são soluções para a equação:

$$
\mathbf{A x}=\lambda \mathbf{B x}, \quad \mathbf{x} \neq 0
$$

para matrizes $\mathbf{A}, \mathbf{B} \in \mathbb{C}^{n x n}, \lambda \in \mathbb{C}$ são os autovalores generalizados e $\mathbf{x}$ são os autovetores generalizados de $\mathbf{A}-\lambda \mathbf{B}$. Os autovalores generalizados são calculados através das raizes $\lambda_{i}$ da função característica:

$$
|\mathbf{A}-\lambda \mathbf{B}|=0
$$

Uma observação importante se faz quando $\operatorname{posto}(\mathbf{B})<n$. Para este caso, o conjunto de autovalores generalizados pode ser finito, vazio ou infinito dependendo da matriz A [14]. Já para posto $(\mathbf{B})=n$ há sempre $n$ autovalores generalizados.

Retornando a eq. (4.22), os $\gamma_{l}$ da matriz $\widetilde{\mathbf{X}}(\gamma)$ são os autovalores generalizados das matrizes $(\mathbf{C}, \mathbf{D})$ :

$$
G E V(\mathbf{C}, \mathbf{D})=\{\gamma \in \mathbb{C}:|\mathbf{C}-\gamma \mathbf{D}|=0\}
$$

$\mathrm{Na}$ prática, tem-se apenas estimativas das matrizes $\mathbf{C}$ e $\mathbf{D}$ calculadas a partir dos dados coletados pelo array, ou seja, as matrizes $\hat{\mathbf{C}}$ e $\hat{\mathbf{D}}$. Eventualmente há situações onde $\hat{\mathbf{D}}$ é mal condicionada e aplicam-se métodos numéricos robustos para determinar os autovalores generalizados. Em geral, os $G E V(\hat{\mathbf{C}}, \hat{\mathbf{D}})$ são calculados através do método numérico QZ [14]. Similar ao método QR para diagonalização de matrizes, o QZ permite a diagonalização simultânea, das matrizes $\hat{\mathbf{C}}$ e $\hat{\mathbf{D}}$ em matrizes diagonais $\hat{\mathbf{C}}^{\prime}=\mathbf{Q} \hat{\mathbf{C Z}}$ e $\hat{\mathbf{D}}^{\prime}=\mathbf{Q} \hat{\mathbf{D} Z}$. Como a matriz $\hat{\widetilde{\mathbf{X}}}(\gamma)=\hat{\mathbf{C}}-\gamma \hat{\mathbf{D}}$ é equivalente a $\hat{\tilde{\mathbf{X}}}^{\prime}(\gamma)=\hat{\mathbf{C}}^{\prime}-\gamma \hat{\mathbf{D}}^{\prime}$, esta última pode ser resolvida sem problemas de cálculo numérico. 
Para finalizar, calculam-se os ângulos estimados $\hat{\theta}_{l}$ a partir dos autovalores generaliza$\operatorname{dos} \hat{\gamma}_{l}$ :

$$
\hat{\theta}_{l}=\arcsin \left(\frac{\arg \left(\hat{\gamma}_{l}\right)}{k|\vec{\Delta}|}\right), \quad l=1,2,3, \cdots, d
$$

Ressalta-se que em todo o algoritmo não é necessário conhecer a geometria do array para determinar a localização das fontes, ou seja a matriz A não foi explicitamente definida. Não é feita varredura como no beamforming clássico, os autovalores generalizados determinam diretamente as posições das fontes. O ESPRIT prevê ainda que além das direções pode-se determinar a potência relativa de cada fonte, a matriz $\mathbf{A}$ que define a geometria dos arrays $X$ e $Y$ através dos dados coletados, e uma reconstrução dos sinais originais de cada fonte [27].

O ESPRIT tem algumas limitações que precisam ser observadas. Sinais de banda estreita são considerados no modelo de sinal, enquanto que, os sinais acústicos são de banda larga. Esta restrição é contornada ao considerar que o ESPRIT é sintonizável em diferentes frequências e podendo dividir o espectro acústico em sub-bandas, com o ESPRIT sendo aplicado em cada frequência central. As fontes cujas localizações são determinas não podem ser correlacionadas, dado que na matriz $\boldsymbol{\Phi}$ fontes correlacionadas ocuparão uma mesma linha, sendo impossível distinguir entre as duas. Para eliminar o ruído na Eq. (4.20), foram calculados os autovalores de $\mathbf{R}_{x x}$. Isto permite determinar a potência do ruído $\sigma^{2}$. A matriz $\mathbf{R}_{x x}$ tem dimensão $m$, e se houver um número de fontes $d \geq m$ torna-se impossível obter a potência do ruído, já que na decomposição por autovalores há no máximo $m$ autovalores. Em outras palavras, o número de sensores do array determina o número máximo de fontes que é possível localizar.

\subsection{D-ESPRIT}

O ESPRIT como apresentado em [27] é limitado a arrays cujos deslocamentos são apenas em uma direção e a localização das fontes é feito por um único parâmetro angular. Em aeroacústica, aplicam-se arrays planares localizando bidimensionalmente as fontes através dos parâmetros de azimute e elevação. Para obter a localização através de 2 parâmetros com o ESPRIT é preciso uma extensão dos conceitos apresentados na Sec. 4.1. Uma possibilidade é a aplicação do ESPRIT para dois vetores de deslocamento ortogonais que forneceriam independentemente o azimute e elevação. No entanto, separando os parâmetros desta forma como 2 aplicações do ESPRIT independentes, haverá uma etapa posterior, a de parear os resultados, i.e. associar os valores de azimute e elevação das fontes. Na Sec. 4.2.1 este problema do pareamento é investigado. A Sec. 4.2.2 introduz a extensão do ESPRIT o 2D-ESPRIT e explica como se faz o pareamento automático. 


\subsubsection{O Problema do Pareamento}

O pareamento não é uma tarefa trivial, e tem diferentes algoritmos descritos em literatura para fazê-lo [8], [31, 332. O pareamento se torna um obstáculo quando são consideradas mais de uma fonte a ser localizada. Para apreciar a vantagem trazida pelo 2D-ESPRIT, o problema do pareamento é explorado. Um exemplo simples em que se deseja localizar no espaço tridimensional 2 fontes distintas localizadas no campo distante segue para ilustrar o problema.

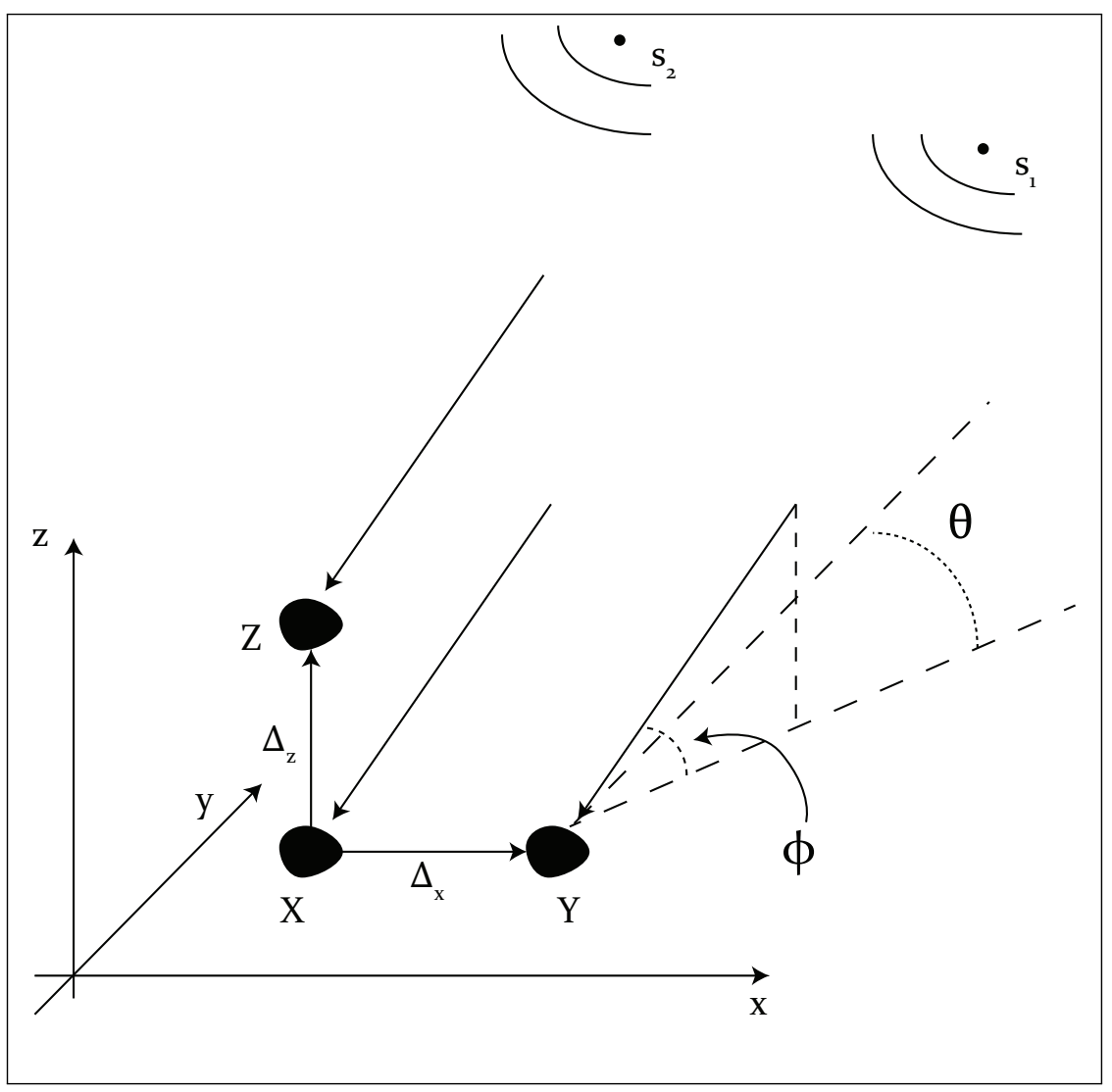

Figura 4.3: Configuração típica para aplicação do ESPRIT em dois vetores de deslocamento. Os arrays estão posicionados sobre o plano $X Z$. A elevação $\phi$ é medida do plano $X Y$ e o azimute $\theta$ é medido sobre o $X Y$ a partir do eixo $y$.

O ESPRIT é aplicado em dois vetores de deslocamento distintos, ilustrados na Fig. 4.3. Os deslocamentos são ortogonais entre si e os 3 arrays são idênticos. As diferenças de fase entre os arrays para uma onda plana atingindo os arrays são descritas por:

$$
u_{l}=|\vec{k}|\left|\vec{\Delta}_{x}\right| \cos \phi_{l} \sin \theta_{l}
$$

para diferença de fase entre os arrays $X$ e $Y$ e

$$
v_{l}=|\vec{k}|\left|\vec{\Delta}_{z}\right| \cos \phi_{l} \cos \theta_{l}
$$

para os arrays $X$ e $Z$. 
São resultados do ESPRIT, aplicado independentemente a cada deslocamento, as estimativas $\hat{u}_{1}, \hat{u}_{2}$ e $\hat{v}_{1}, \hat{v}_{2}$. Como não há conhecimento da posição das fontes, um pareamento das estimativas $(u, v)$ para identificar precisamente as posições faz-se necessário. Há duas possibilidades para este exemplo didático, os pares $\left(\hat{u}_{1}, \hat{v}_{1}\right)$ e $\left(\hat{u}_{2}, \hat{v}_{2}\right)$ ou os pares $\left(\hat{u}_{1}, \hat{v}_{2}\right)$ e $\left(\hat{u}_{2}, \hat{v}_{1}\right)$. Um critério para determinar a combinação correta deve ser definido. No 2D-ESPRIT, esta etapa de escolha é dispensada calculando simultâneamente os parâmetros e pareando-os automaticamente. O resultado da aplicação do 2D-ESPRIT sobre os dados coletados do array são pares de parâmetros associados a cada uma das fontes.

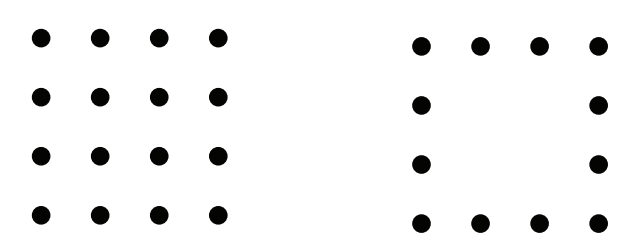

Figura 4.4: Exemplos de arrays com centro simetria utilizáveis no 2D-ESPRIT.

\subsubsection{D-ESPRIT como Solução do Pareamento}

O algoritmo 2D Unitary ESPRIT [16], ou simplesmente 2D-ESPRIT, apresenta uma solução prática para o pareamento que é computacionalmente eficiente e sem *precisar* de cálculos complicados como os vistos em [8] que utiliza soluções de sistemas não lineares. O 2D-ESPRIT requer um array cuja geometria é centro simétrica e que tenha dupla invariância. A geometria centro simétrica é aquela em que os $M$ sensores do array são posicionados aos pares em volta de um centro geométrico ou centróide. Uma estrutura de dupla invariância é uma geometria onde existe um par de subarrays idênticos de $m_{x}$ sensores deslocados no eixo $X$ e outro par de subarrays idênticos de $m_{z}$ sensores deslocados no eixo $Z$. Alguns exemplos estão ilustrados na Fig. 4.4. Em particular, o array quadrado de 4x4 sensores satisfaz as propriedades e é usado na implementação empregada aqui.

Na Fig. 4.5, o array quadrado $4 \mathrm{x} 4$ apresenta seus subarrays no eixo $X$ à esquerda e os subarrays no eixo $Z$ à direita. Para identificar cada subarray definem-se matrizes de seleção $J_{x 1}, J_{x 2}, J_{z 1}$ e $J_{z 2}$. Estas matrizes são binárias identificando quais os elementos do array pertencem ao subarray. Devido a centro simetria do array as matrizes de seleção de uma mesma invariância estão relacionados por:

$$
\mathbf{J}_{x 2}=\boldsymbol{\Pi}_{m x} \mathbf{J}_{x 1} \boldsymbol{\Pi}_{M}
$$

e

$$
\mathbf{J}_{z 2}=\Pi_{m z} \mathbf{J}_{z 1} \boldsymbol{\Pi}_{M}
$$




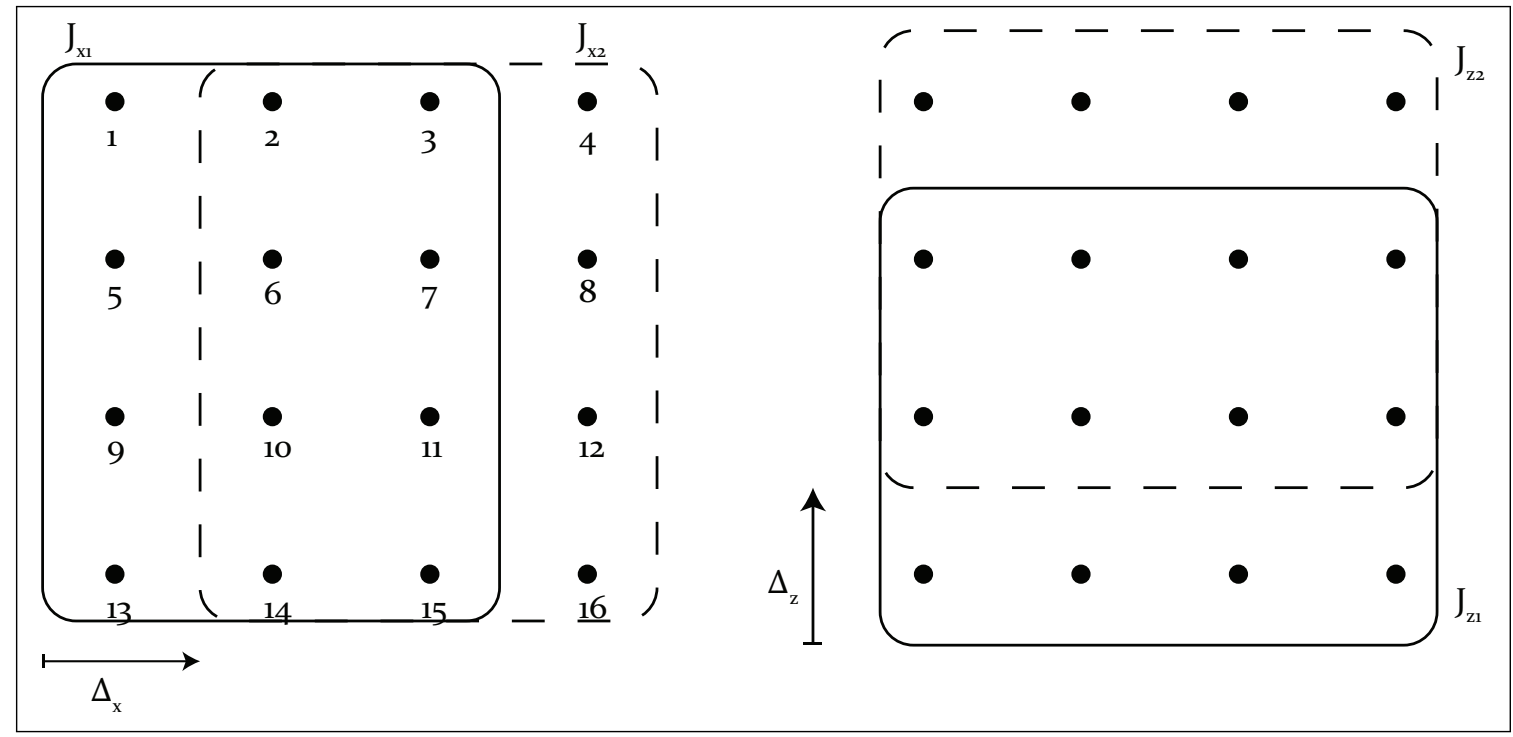

Figura 4.5: Array quadrado com 16 elementos dispostos uniformemente $4 \mathrm{x} 4$ sob o plano $X Z$. No lado esquerdo, os sub-arrays idênticos que tem vetor de deslocamento $\vec{\Delta}_{x}$ e do lado direito, os sub-arrays que tem vetor de deslocamento $\vec{\Delta}_{z}$.

onde $\Pi_{n}$ é a matriz de permutação que troca a ordem das linhas ou colunas.

$$
\Pi_{n}=\left[\begin{array}{cccccc}
0 & 0 & \cdots & 0 & 0 & 1 \\
0 & 0 & \cdots & 0 & 1 & 0 \\
0 & 0 & \cdots & 1 & 0 & 0 \\
\vdots & \vdots & \cdots & \vdots & \vdots & \vdots \\
0 & 1 & \cdots & 0 & 0 & 0 \\
1 & 0 & \cdots & 0 & 0 & 0
\end{array}\right]_{n x n}
$$

Como exemplo considere o array 2x2 da Fig. 4.6. Com a numeração dada pela figura, a matriz de seleção do primeiro subarray do eixo $X$ é:

$$
\mathbf{J}_{x 1}=\left[\begin{array}{llll}
1 & 0 & 0 & 0 \\
0 & 0 & 1 & 0
\end{array}\right]
$$

que indica que os sensores 1 e 3 participam do subarray $x 1$. Para o segundo subarray no eixo $X$, a matriz de seleção é:

$$
\mathbf{J}_{x 2}=\left[\begin{array}{llll}
0 & 1 & 0 & 0 \\
0 & 0 & 0 & 1
\end{array}\right]
$$

ou seja, os sensores 2 e 4 estão no subarray $x 2$. Devido à centro simetria do array, verifica-se 


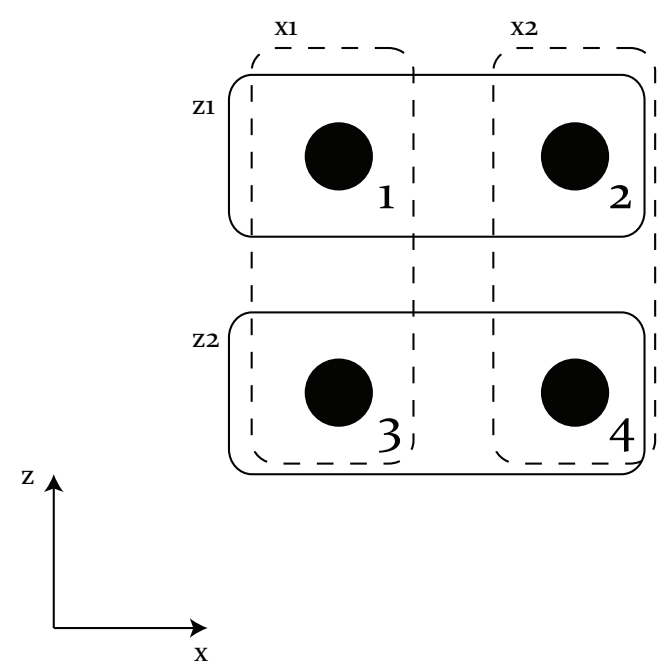

Figura 4.6: Exemplo da aplicação das matrizes de seleção para os subarrays $J_{x 1}, J_{x 2}, J_{z 1}$ e $J_{z 2}$, em um array $2 \times 2$ quadrado com elementos uniformemente espaçados.

que:

$$
\begin{aligned}
\mathbf{J}_{x 2} & =\Pi_{m x} \mathbf{J}_{x 1} \boldsymbol{\Pi}_{M} \\
& =\left[\begin{array}{ll}
0 & 1 \\
1 & 0
\end{array}\right]\left[\begin{array}{llll}
1 & 0 & 0 & 0 \\
0 & 0 & 1 & 0
\end{array}\right]\left[\begin{array}{llll}
0 & 0 & 0 & 1 \\
0 & 0 & 1 & 0 \\
0 & 1 & 0 & 0 \\
1 & 0 & 0 & 0
\end{array}\right] \\
& =\left[\begin{array}{llll}
0 & 1 & 0 & 0 \\
0 & 0 & 0 & 1
\end{array}\right]
\end{aligned}
$$

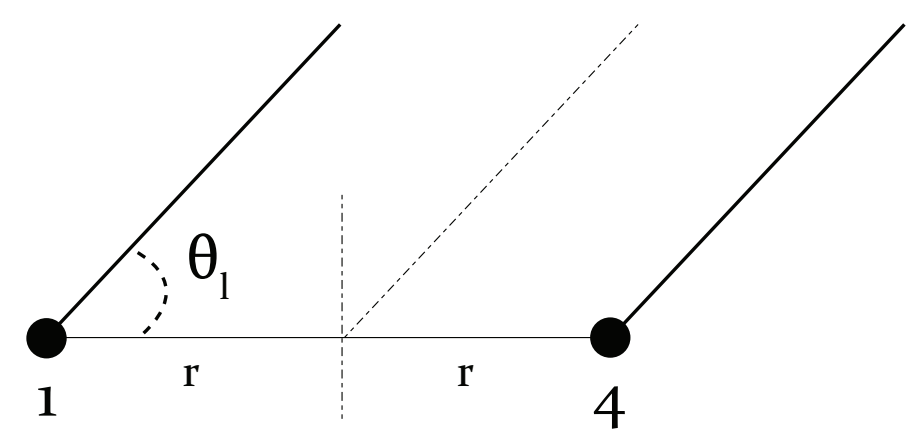

Figura 4.7: Par de sensores 1-4 do array 2x2 em destaque. Ambos distam do centróide de $r$, que permite relacionar a fase da onda plana que atinge os sensores através do conjugado complexo.

Uma consequência da geometria centro simétrica do array é que os sinais recebidos pelos pares de sensores posicionados ao redor do centroide estão relacionados. Uma onda plana qualquer atingindo um par de sensores localizados sobre o plano XZ, formando um ângulo $\theta_{l}$, tem fase adiantada em um dos sensores e tem fase igualmente atrasada no outro 
sensor, sempre tomando o centro geométrico do par de sensores como referência. A Fig. 4.7 ilustra este arranjo. Os valores das fases são devidos à escolha do centro entre os sensores 1 e 4 como referência. O "steering vector" para uma fonte neste exemplo unidimensional é dado por:

$$
\mathbf{a}\left(\theta_{l}\right)=\left[\begin{array}{l}
a_{1}\left(\theta_{l}\right) \\
a_{4}\left(\theta_{l}\right)
\end{array}\right]=\left[\begin{array}{c}
e^{-j k r \sin \left(\theta_{l}\right)} \\
e^{j k r \sin \left(\theta_{l}\right)}
\end{array}\right]
$$

Os $a_{i}\left(\theta_{l}\right)$ são os fatores que multiplicam os sinais recebidos efetuando os atrasos necessários para poder orientar o array para uma direção desejada. Como observado na Eq. (4.37) os $a_{i}\left(\theta_{l}\right)$ dos pares estão relacionados:

$$
a_{1}\left(\theta_{l}\right)=\bar{a}_{4}\left(\theta_{l}\right)
$$

em que a barra indica o complexo conjugado.

No caso de arrays planares os "steering vectors" têm dependência adicional da elevação $a_{i}\left(\theta_{l}, \phi_{l}\right)$ e os pares obedecem a seguinte relação:

$$
a_{k}^{\prime}\left(\theta_{l}, \phi_{l}\right)=\bar{a}_{k}^{\prime \prime}\left(\theta_{l}, \phi_{l}\right) \quad k=1,2, \cdots, M / 2
$$

Juntando todos os "steering vectors" para cada fonte em uma matriz tem-se:

$$
\begin{aligned}
\mathbf{A}_{T} & =\left[\begin{array}{cccc}
a_{1}\left(\theta_{1}, \phi_{1}\right) & a_{1}\left(\theta_{2}, \phi_{2}\right) & \cdots & a_{1}\left(\theta_{d}, \phi_{d}\right) \\
a_{2}\left(\theta_{1}, \phi_{1}\right) & a_{2}\left(\theta_{2}, \phi_{2}\right) & \cdots & a_{2}\left(\theta_{d}, \phi_{d}\right) \\
\vdots & \vdots & & \vdots \\
a_{M}\left(\theta_{1}, \phi_{1}\right) & a_{M}\left(\theta_{2}, \phi_{2}\right) & \cdots & a_{M}\left(\theta_{d}, \phi_{d}\right)
\end{array}\right] \\
& =\left[\begin{array}{llll}
\mathbf{a}\left(\theta_{1}, \phi_{1}\right) & \mathbf{a}\left(\theta_{2}, \phi_{2}\right) & \cdots & \mathbf{a}\left(\theta_{d}, \phi_{d}\right)
\end{array}\right]
\end{aligned}
$$

Procura-se manter a simetria exibida pelo array nos "steering vectors", mantendo os elementos dos pares centro hermitanos em linhas opostas:

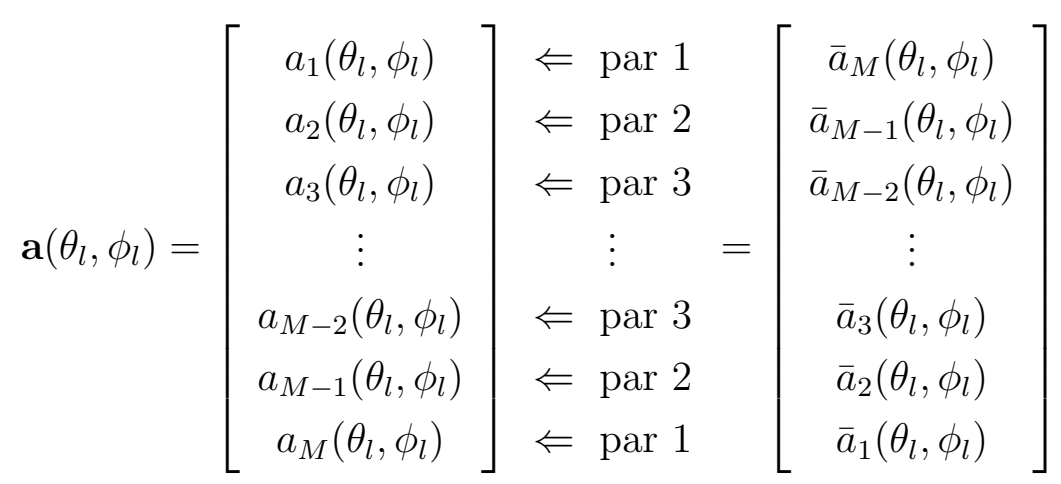


Com esta construção, a matriz $\mathbf{A}_{T}$ contém vetores coluna centro hermitianos e vale:

$$
\mathbf{A}_{T}=\Pi_{M} \overline{\mathbf{A}}_{T}
$$

Ao calcular as matrizes de covariância $\mathbf{C}_{x}$ e covariância cruzada $\mathbf{D}_{x}$ como feito para o ESPRIT, a geometria centro simétrica resulta em matrizes $\mathbf{C}_{x}^{\prime}$ e $\mathbf{D}_{x}^{\prime}$ que são centro hermitianas. Uma matriz $\mathbf{M} \in \mathbb{C}^{n x m}$ é centro hermitiana se $\Pi_{n} \overline{\mathbf{M}}_{m}=\mathbf{M}$. Da mesma forma, estimam-se as matrizes $\mathbf{C}_{z}^{\prime}$ e $\mathbf{D}_{z}^{\prime}$ para o eixo $Z$. No entanto, as equações matriciais que permitem determinar $\gamma_{x}$ e $\gamma_{z}$ :

$$
\begin{aligned}
\widetilde{X}\left(\gamma_{x}\right) & =\mathbf{C}_{x}^{\prime}-\gamma_{x} \mathbf{D}_{x}^{\prime} \\
\widetilde{Z}\left(\gamma_{z}\right) & =\mathbf{C}_{z}^{\prime}-\gamma_{z} \mathbf{D}_{z}^{\prime}
\end{aligned}
$$

não possuem os mesmos autovetores generalizados impossibilitando o pareamento automático. Em geral, as Eqs. (4.43) e (4.44) tem autovalores $\gamma_{x, l} \neq \gamma_{z, l}$ e autovetores $\mathbf{v}_{x, l}$ que podem ser mapeados para os autovetores $\mathbf{v}_{z, l}$. Os autovalores $\gamma_{x, l}$ e $\gamma_{z, l}$ são pareados já que para cada autovetor está associado um autovalon! 1 .

O teorema descrito em 21] junto com a geometria centro simétrica permitem o pareamento automático sem precisar definir o mapeamento entre os autovetores $\mathbf{v}_{x, l}$ e $\mathbf{v}_{z, l}$. O teorema mencionado realiza um mapeamento bijetor que transforma matrizes centro hermitianas $\mathbf{M} \in \mathbb{C}^{m x n}$ em matrizes $\mathbf{R} \in \mathbb{R}^{m x n}$.

$$
f: \mathbf{M} \mapsto \mathbf{Q}_{m}^{H} \mathbf{M Q}_{n} \in \mathbb{R}^{m x n}
$$

Assim qualquer matriz $\mathbf{Q} \in \mathbb{C}^{m x n}$ unitária que obedeça a relação $\Pi_{m} \overline{\mathbf{Q}}=\mathbf{Q}$, isto é, seja centro hermitiana, realiza um mapeamento semelhante ao da Eq. (4.45). Neste contexto, é usual definir a matriz $\mathbf{Q}$ como:

$$
\mathbf{Q}_{2 p+1}=\frac{1}{\sqrt{2}}\left[\begin{array}{ccc}
\mathbf{I}_{p} & \mathbf{0} & j \mathbf{I}_{p} \\
\mathbf{0}^{T} & \sqrt{2} & \mathbf{0}^{T} \\
\boldsymbol{\Pi}_{p} & \mathbf{0} & -j \boldsymbol{\Pi}_{p}
\end{array}\right]
$$

para $\mathbf{Q}$ com dimensão ímpar, e

$$
\mathbf{Q}_{2 p}=\frac{1}{\sqrt{2}}\left[\begin{array}{cc}
\mathbf{I}_{p} & j \mathbf{I}_{p} \\
\boldsymbol{\Pi}_{p} & -j \boldsymbol{\Pi}_{p}
\end{array}\right]
$$

para dimensão par.

Para um array centro simétrico qualquer e com matriz de "steering vectors" $\mathbf{A}_{T} \in \mathbb{C}^{M x d}$

\footnotetext{
${ }^{1}$ Se as amplitudes de cada fonte fossem diferentes seria óbvio o pareamento já que seria possível ordenar os autovalores generalizados $\gamma_{x}$ e $\gamma z$ e associá-los as amplitudes. No entanto, com amplitudes iguais ou até mesmo com valores próximos, esta ordenação não permite associar os pares às fontes.
} 
aplica-se a transformação em cada vetor através da equação:

$$
\mathbf{Q}^{H} \mathbf{A}_{T}=\mathbf{G}
$$

em que $\mathbf{G} \in \mathbb{R}^{M x d}$.

Os subarrays no eixo $X$ estão relacionados por:

$$
\mathbf{J}_{x 1} \mathbf{A}_{T} \boldsymbol{\Phi}_{x}=\mathbf{J}_{x 2} \mathbf{A}_{T}
$$

em que $\boldsymbol{\Phi}_{x}$ é matriz diagonal contendo as direções das $d$ fontes que deseja-se determinar.

$$
\boldsymbol{\Phi}_{x}=\left[\begin{array}{ccccc}
e^{j u_{1}} & 0 & 0 & \cdots & 0 \\
0 & e^{j u_{2}} & 0 & \cdots & 0 \\
0 & 0 & e^{j u_{2}} & \cdots & 0 \\
& & & \ddots & \\
0 & 0 & 0 & \cdots & e^{j u_{d}}
\end{array}\right]
$$

com $u_{l}, \quad l=1,2, \cdots, d$ definido na Eq. (4.30). Como Q é unitária:

$$
\begin{aligned}
\mathbf{J}_{x 1} \mathbf{Q}_{M} \mathbf{Q}_{M}^{H} \mathbf{A}^{T} \boldsymbol{\Phi}_{x} & =\mathbf{J}_{x 2} \mathbf{Q}_{M} \mathbf{Q}_{M}^{H} \mathbf{A}_{T} \\
\mathbf{J}_{x 1} \mathbf{Q}_{M} \mathbf{G} \boldsymbol{\Phi}_{x} & =\mathbf{J}_{x 2} \mathbf{Q}_{M} \mathbf{G}
\end{aligned}
$$

Como as matrizes de seleção $\mathbf{J}_{x 1}, \mathbf{J}_{x 2}$ satifazem $\mathbf{J}_{x 2}=\mathbf{\Pi} \mathbf{J}_{x 1} \boldsymbol{\Pi}$, então aplicando esta igualdade e multiplicando por $\mathbf{Q}_{m x}^{H}$ pela esquerda na eq. (4.52):

$$
\begin{aligned}
\mathbf{Q}_{m x}^{H} \mathbf{J}_{x 1} \mathbf{Q}_{M} \mathbf{G} \boldsymbol{\Phi}_{x} & =\mathbf{Q}_{m x}^{H} \mathbf{J}_{x 2} \mathbf{Q}_{M} \mathbf{G} \\
\mathbf{Q}_{m x}^{H} \mathbf{J}_{x 1} \mathbf{Q}_{M} \mathbf{G} \boldsymbol{\Phi}_{x} & =\mathbf{Q}_{m x}^{H} \Pi_{m x} \underbrace{\Pi_{m x} \mathbf{J}_{x 2} \Pi_{M}}_{\mathbf{J}_{x 1}} \Pi_{M} \mathbf{Q}_{M} \mathbf{G} \\
\mathbf{Q}_{m x}^{H} \mathbf{J}_{x 1} \mathbf{Q}_{M} \mathbf{G} \boldsymbol{\Phi}_{x} & =\underbrace{\mathbf{Q}_{m x}^{H} \boldsymbol{\Pi}_{m x}}_{\mathbf{Q}_{m x}^{T}} \mathbf{J}_{x 1} \underbrace{\Pi_{M} \mathbf{Q}_{M}}_{\mathbf{Q}_{M}^{*}} \mathbf{G} \\
\mathbf{Q}_{m x}^{H} \mathbf{J}_{x 1} \mathbf{Q}_{M} \mathbf{G} \boldsymbol{\Phi}_{x} & =\mathbf{Q}_{m x}^{T} \mathbf{J}_{x 1} \mathbf{Q}_{M}^{*} \mathbf{G} \\
\mathbf{Q}_{m x}^{H} \mathbf{J}_{x 1} \mathbf{Q}_{M} \mathbf{G} \boldsymbol{\Phi}_{x} & =\left(\mathbf{Q}_{m x}^{H} \mathbf{J}_{x 1} \mathbf{Q}_{M}\right)^{*} \mathbf{G}
\end{aligned}
$$

em que foram utilizadas as identidades $\Pi \Pi=\mathbf{I}$ e $\Pi \mathbf{\Pi}=\mathbf{Q}^{*}$. A substituição a seguir, resulta em um mapeamento simples dos autovalores do domínio dos reais para os autovalores originais no domínio dos complexos. Definem-se as matrizes:

$$
\begin{aligned}
\mathbf{K}_{x 1} & =\operatorname{Re}\left\{\mathbf{Q}_{m x}^{H} \mathbf{J}_{x 1} \mathbf{Q}_{M}\right\} \\
\mathbf{K}_{x 2} & =\operatorname{Im}\left\{\mathbf{Q}_{m x}^{H} \mathbf{J}_{x 1} \mathbf{Q}_{M}\right\}
\end{aligned}
$$


e substituindo na eq. (4.57),

$$
\left(\mathbf{K}_{x 1}+j \mathbf{K}_{x 2}\right) \mathbf{G} \boldsymbol{\Phi}_{x}=\left(\mathbf{K}_{x 1}-j \mathbf{K}_{x 2}\right) \mathbf{G}
$$

Pode-se fatorar a matriz $\boldsymbol{\Phi}_{x}$ da seguinte maneira:

$$
\Phi_{x}=\Phi_{x / 2} \Phi_{x / 2}
$$

em que

$$
\boldsymbol{\Phi}_{x / 2}=\left[\begin{array}{ccccc}
e^{j \frac{u_{1}}{2}} & 0 & 0 & \cdots & 0 \\
0 & e^{j \frac{u_{2}}{2}} & 0 & \cdots & 0 \\
0 & 0 & e^{j \frac{u_{3}}{2}} & \cdots & 0 \\
\vdots & \vdots & \vdots & \ddots & \vdots \\
0 & 0 & 0 & \cdots & e^{j \frac{u_{d}}{2}}
\end{array}\right]
$$

Retornando a Eq. (4.60) pode-se re-escrevê-la como:

$$
\begin{gathered}
\left(\mathbf{K}_{x 1}+j \mathbf{K}_{x 2}\right) \mathbf{G} \boldsymbol{\Phi}_{x / 2} \boldsymbol{\Phi}_{x / 2}=\left(\mathbf{K}_{x 1}-j \mathbf{K}_{x 2}\right) \mathbf{G} \\
\left(\mathbf{K}_{x 1}+j \mathbf{K}_{x 2}\right) \mathbf{G} \boldsymbol{\Phi}_{x / 2}=\left(\mathbf{K}_{x 1}-j \mathbf{K}_{x 2}\right) \mathbf{G} \boldsymbol{\Phi}_{x / 2}^{H} \\
\mathbf{K}_{x 1} \mathbf{G}\left(\boldsymbol{\Phi}_{x / 2}-\boldsymbol{\Phi}_{x / 2}^{H}\right)=-j \mathbf{K}_{x 2} \mathbf{G}\left(\boldsymbol{\Phi}_{x / 2}+\boldsymbol{\Phi}_{x / 2}^{H}\right)
\end{gathered}
$$

em que $\boldsymbol{\Phi}_{x / 2} \boldsymbol{\Phi}_{x / 2}^{H}=$ I. Já que $\left(\boldsymbol{\Phi}_{x / 2}+\boldsymbol{\Phi}_{x / 2}^{H}\right)$ continua sendo uma matriz diagonal na Eq. (4.65) então vale:

$$
\left(\boldsymbol{\Phi}_{x / 2}+\boldsymbol{\Phi}_{x / 2}^{H}\right)\left(\boldsymbol{\Phi}_{x / 2}+\boldsymbol{\Phi}_{x / 2}^{H}\right)^{-1}=\mathbf{I}
$$

e na Eq. (4.65),

$$
\begin{aligned}
\mathbf{K}_{x 1} \mathbf{G} \underbrace{\frac{-1}{j}\left(\boldsymbol{\Phi}_{x / 2}-\boldsymbol{\Phi}_{x / 2}^{H}\right)\left(\boldsymbol{\Phi}_{x / 2}+\boldsymbol{\Phi}_{x / 2}^{H}\right)^{-1}}_{\mathbf{\Psi}_{x}} & =\mathbf{K}_{x 2} \mathbf{G} \\
\mathbf{K}_{x 1} \mathbf{G} \boldsymbol{\Psi}_{x} & =\mathbf{K}_{x 2} \mathbf{G}
\end{aligned}
$$


A matriz $\Psi_{x}$ pode ser simplificada utilizando a equação de Euler $e^{j x}=\cos (x)+j \sin (x)$ :

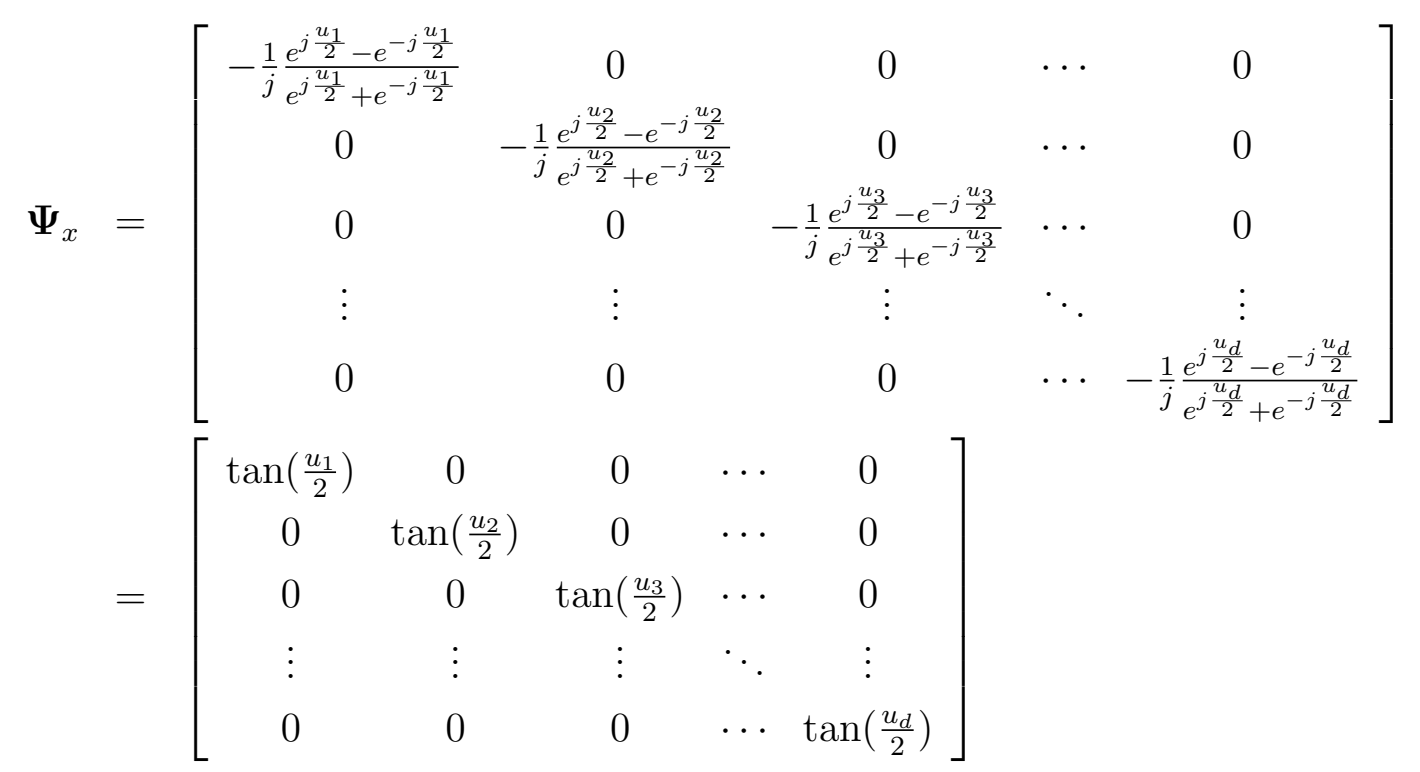

que é uma matriz real $\Psi_{x} \in \mathbb{R}^{d x d}$. As tangentes na matriz $\boldsymbol{\Psi}_{x}$ são resultado da substituição feita com as matrizes $\mathbf{K}_{1}$ e $\mathbf{K}_{2}$. A Eq. (4.68) indica que pode-se determinar a localização da fonte través do mapeamento da Eq. (4.45). O mesmo raciocinio é feito para obter a equação matricial para o eixo $Z$ :

$$
\mathbf{K}_{z 1} \mathbf{G} \Psi_{z}=\mathbf{K}_{z 2} \mathbf{G}
$$

A matriz G está presente nas duas equações, já que é representação dos "steering vectors" de todo o array no domínio dos reais.

Até este momento não foram considerados os dados coletados do array. Assim como no ESPRIT, o 2D-ESPRIT considera dois subespaços vetoriais de sinal e de ruído. Esta separação permite uma rejeição ao ruído melhor do que o beamforming clássico, vista no Cap. 5. Há diversas maneiras de construir o subespaço de sinal no 2D-ESPRIT. A mais intuitiva é obter a estimativa da matriz de covariância $\hat{\mathbf{R}}_{T}$ de todo o array (as matrizes de seleção são utilizadas para cada subarray), e mapeá-la para o domínio dos reais utilizando as matrizes unitárias Q. Como a estimativa da matriz de covariância não é exatamente centrohermitiana, devemos garantir que o resultado do mapeamento seja real tomando apenas as parcelas reais assim:

$$
\hat{R}_{Q Q}=\operatorname{Re}\left\{\mathbf{Q}^{H} \hat{\mathbf{R}}_{T} \mathbf{Q}\right\}
$$

A decomposição em autovalores da Eq. (4.72), separam-se os $d$ maiores autovalores referentes a cada fonte, e seus respectivos autovetores $\mathbf{e}_{l}$. Os autovetores são agrupados na matriz $\mathbf{E}_{s}$, que representa os sinais projetados sobre o espaço vetorial do array.

$$
\mathbf{E}_{s}=\left[\begin{array}{llll}
\mathbf{e}_{1} & \mathbf{e}_{2} & \cdots & \mathbf{e}_{d}
\end{array}\right]
$$


Outra forma sugerida por [16] para obter a matriz $\mathbf{E}_{s}$ é através da decomposição em valores singulares (SVD) da matriz de dados expandida, definida para ser centro hermitiana:

$$
\left[\begin{array}{ll}
\mathbf{X} & \Pi \mathbf{X} \Pi
\end{array}\right]
$$

em que cada coluna da matriz $\mathbf{X}$ contém as amostras coletadas em todos os sensores do array para um intante $t$.

A matriz $\mathbf{G}$ nas Eqs. (4.68) e (4.71) está relacionada com a matriz $\mathbf{E}_{s}$ por:

$$
\mathbf{E}_{s}=\mathbf{G T}
$$

em que T é uma matriz desconhecida que multiplicada pelos "steering vectors" do array, obtém-se os vetores dos sinais recebidos. Isto é verdade já que, como visto no Cap. 3, para conseguir os vetores de sinais em $\mathbf{E}_{s}$ é necessário utilizar os "steering vectors".

Como $\mathbf{E}_{s}$ e $\mathbf{G}$ são de posto completo vale:

$$
\mathbf{G}=\mathbf{E}_{s} \mathbf{T}^{-1}
$$

e aplicando esta relação na Eq. (4.68),

$$
\begin{aligned}
\mathbf{K}_{x 1} \mathbf{E}_{s} \mathbf{T}^{-1} \boldsymbol{\Psi}_{x} & =\mathbf{K}_{x 2} \mathbf{E}_{s} \mathbf{T}^{-1} \\
\mathbf{K}_{x 1} \mathbf{E}_{s} \underbrace{\mathbf{T}^{-1} \boldsymbol{\Psi}_{x} \mathbf{T}}_{\boldsymbol{\Gamma}_{x}} & =\mathbf{K}_{x 2} \mathbf{E}_{s}
\end{aligned}
$$

e para a Eq. (4.71)

$$
\mathbf{K}_{z 1} \mathbf{E}_{s} \underbrace{\mathbf{T}^{-1} \boldsymbol{\Psi}_{z} \mathbf{T}}_{\boldsymbol{\Gamma}_{x}}=\mathbf{K}_{z 2} \mathbf{E}_{s}
$$

Tanto a matriz $\boldsymbol{\Gamma}_{x}$ quanto a matriz $\boldsymbol{\Gamma}_{z}$ são reais e tal que $\boldsymbol{\Gamma}_{x}, \boldsymbol{\Gamma}_{z} \in \mathbb{R}^{d x d}$, já que aqui as equações do ESPRIT estão todas no domínio dos reais feitas através do mapeamento $f$. Em ambas as Eqs. (4.78) e (4.79), as matrizes $\boldsymbol{\Gamma}_{x}$ e $\boldsymbol{\Gamma}_{z}$ são calculadas através de mínimos quadrados. Com estas matrizes determinadas, nota-se que $\boldsymbol{\Gamma}_{x}$ e $\boldsymbol{\Gamma}_{z}$ são decomposições em autovalores (diagonal de $\Psi_{x}$ e $\Psi_{z}$ ) e em autovetores $\mathbf{T}$ os quais ambas matrizes compartilham. Estes autovetores que são utilizados em ambas as decomposições são aqueles que permitem o pareamento automático, que é impossível de ser realizado no domínio complexo. A decomposição para determinar os $\mathbf{T}$ 's e as diagonais de $\boldsymbol{\Psi}_{x}$ e $\boldsymbol{\Psi}_{z}$ deve ser feita simultaneamente. O cálculo simlutâneo é feito levando em consideração que todas as matrizes estão no domínio dos reais e portanto é válido construír a matriz complexa a partir das matrizes $\boldsymbol{\Gamma}_{x}$ e $\boldsymbol{\Gamma}_{z}$ :

$$
\boldsymbol{\Gamma}_{x}+j \boldsymbol{\Gamma}_{z}
$$


cuja decomposição em autovalores e autovetores é:

$$
\boldsymbol{\Gamma}_{x}+j \boldsymbol{\Gamma}_{z}=\mathbf{T} \underbrace{\left(\boldsymbol{\Psi}_{x}+j \boldsymbol{\Psi}_{z}\right)}_{\Omega_{\text {pair }}} \mathbf{T}^{-1}
$$

Ao calcular os autovalores e autovetores como na Eq. (4.81) acima pareamos os autovalores contidos nas matrizes $\boldsymbol{\Psi}_{x}$ e $\boldsymbol{\Psi}_{z}$. Como a decomposição gerou uma matriz complexa $\Omega_{\text {pair }}$, extraem-se os autovalores $\psi_{x, l}$ e $\psi_{z, l}$ por:

$$
\begin{aligned}
& \Psi_{x}=\operatorname{diag}\left\{\psi_{x, l}, l=1,2,3, \cdots, d\right\}=\operatorname{Re}\left\{\Omega_{\text {pair }}\right\} \\
& \Psi_{z}=\operatorname{diag}\left\{\psi_{z, l}, l=1,2,3, \cdots, d\right\}=\operatorname{Im}\left\{\Omega_{\text {pair }}\right\}
\end{aligned}
$$

em que a diagonal principal de $\boldsymbol{\Psi}_{x}$ e $\boldsymbol{\Psi}_{z}$ contém, os autovalores ordenados $\psi_{x, l}$ e $\psi_{z, l}$. Por fim calculam-se as fases $u_{l}$ e $v_{l}$ sem voltar ao domínio dos reais, já que:

$$
\begin{aligned}
& u_{l}=2 \arctan \left(\psi_{x, l}\right) \\
& v_{l}=2 \arctan \left(\psi_{z, l}\right)
\end{aligned}
$$

Resume-se o 2D-ESPRIT na seção a seguir.

\section{Resumo do 2D-ESPRIT}

Resumindo o algoritmo é composto pelos seguintes passos:

1. Construir a matriz $\mathbf{E}_{s}$ com os $d$ maiores autovetores da decomposição de $\operatorname{Re}\left\{\mathbf{Q}^{H} \mathbf{R}_{T} \mathbf{Q}\right\}$.

2. Com

$$
\begin{gathered}
\mathbf{K}_{x 1}=\mathbf{Q}_{m x}^{H}\left(\mathbf{J}_{x 1}+\mathbf{J}_{x 2}\right) \mathbf{Q}_{M} \\
\mathbf{K}_{x 2}=\mathbf{Q}_{m x}^{H} j\left(\mathbf{J}_{x 1}-\mathbf{J}_{x 2}\right) \mathbf{Q}_{M}
\end{gathered}
$$

e

$$
\begin{aligned}
& \mathbf{K}_{z 1}=\mathbf{Q}_{m z}^{H}\left(\mathbf{J} \mathbf{z} \mathbf{1}+\mathbf{J}_{\mathbf{z} 2}\right) \mathbf{Q}_{M} \\
& \mathbf{K}_{z 2}=\mathbf{Q}_{m x}^{H} j\left(\mathbf{J}_{z 1}-\mathbf{J}_{z 2}\right) \mathbf{Q}_{M}
\end{aligned}
$$

calculam-se as matrizes $\boldsymbol{\Gamma}_{x}$ e $\boldsymbol{\Gamma}_{z}$ através de mínimos quadrados as equações:

$$
\begin{aligned}
\mathbf{K}_{x 1} \mathbf{E}_{s} \boldsymbol{\Gamma}_{x} & =\mathbf{K}_{x 2} \mathbf{E}_{s} \\
\mathbf{K}_{z 1} \mathbf{E}_{s} \boldsymbol{\Gamma}_{z} & =\mathbf{K}_{z 2} \mathbf{E}_{s}
\end{aligned}
$$


3. As matrizes $\boldsymbol{\Gamma}_{x}$ e $\boldsymbol{\Gamma}_{z}$ são decompostas simultaneamente através da equação:

$$
\boldsymbol{\Gamma}_{x}+j \boldsymbol{\Gamma}_{z}=\mathbf{T}\left(\boldsymbol{\Psi}_{x}+\mathbf{\Psi}_{z}\right) \mathbf{T}^{-1}
$$

4. Os autovalores da decomposição presentes nas diagonais das matrizes $\boldsymbol{\Psi}_{x}=\operatorname{diag}\left\{\tan \left(u_{l}\right) / 2\right\}$ e $\boldsymbol{\Psi}_{z}=\operatorname{diag}\left\{\tan \left(v_{l}\right) / 2\right\}$ são os pares de cada fonte $l$.

\subsubsection{Comentários Finais}

O algoritmo 2D-ESPRIT resolve o problema do pareamento através da restrição a geometria do array e a aplicação do teorema [21] que aplica o mapeamento descrito na Eq. (4.45). A aplicação do mapeamento possibilitou encontrar autovetores comuns entre as duas invariâncias, fundamental para que haja um pareamento automático entre os parâmetros de azimute e elevação. No entanto, o mapeamento não pode ser aplicado a qualquer geometria do array. Arrays cuja geometria é centro simetrica têm matrizes de "steering vectors" centro hermitianas. As matrizes centro hermitianas por sua vez são aquelas que validam o mapeamento. O ESPRIT como descrito na Sec. 4.1 continua presente como evidenciado nas Eqs. (4.68) e (4.71). Diferente do ESPRIT, todos os cálculos são feitos no domínio dos reais, resultando em um ganho computacional. O 2D-ESPRIT apresenta a solução para o pareamento com simplicidade, favorecendo uma implementação eficiente. As vantagens do ESPRIT são herdados, fazendo do 2D-ESPRIT um algoritmo rápido e utilizando poucas amostras para obter altas resoluções. No entanto, o 2D-ESPRIT adquire desvantagens do ESPRIT como a impossibilidade de fontes correlacionadas e que os sinais a serem identificados são de banda estreita. Mesmo assim, o 2D-ESPRIT é útil para identificar fontes na estrutura de uma aeronave, localizando-as com maior precisão. Na Sec. 5, a implementação do 2D-ESPRIT se mostra extremamente útil mesmo com as restrições descritas. Algoritmos de alta-resolução mostram como é possível retirar o máximo de informação de dados captados de um array. 


\section{Capítulo 5}

\section{Resultados}

Os resultados apresentados a seguir, são consequências de estudos feitos através de simulador. Foram desenvolvidos em MatLab ${ }^{\circledR}$, softwares de simulação para beamforming clássico e 2D-ESPRIT. Para reduzir o custo do estudo desenvolveu-se também um gerador de sinais recebidos pelo array. Neste gerador, uma fonte pontual tonal é colocada em qualquer posição e o gerador tem como saída os sinais de cada microfone de um array. Os sinais gerados desta forma servem de entrada para os algoritmos desenvolvidos.

O beamforming clássico, por ser uma ferramenta de análise comum em processamento de sinais em arrays, foi implementado para comparar o desempenho do 2D-ESPRIT. Houve esforço considerável para que a implementação do beamforming clássico servisse como referência confiável. A Sec. 5.1 apresenta os resultados de verificação do algoritmo de beamforming clássico. Na Sec. [5.2, os algoritmos beamforming clássico e 2D-ESPRIT são comparados para alguns casos de interesse. Nesta mesma seção as características de alta resolução e rejeição ao ruído são verificadas, apresentando o 2D-ESPRIT como uma alternativa com melhor desempenho.

\subsection{Beamforming Clássico}

Na Fig. 5.1, a geometria para validação do algoritmo de beamforming clássico é composta por um array de 4 sensores identificados pelos círculos vermelhos e uma fonte pontual tonal localizada no ' $x$ ' em azul. As Figs. 5.2 e 5.3 apresentam as imagens acústicas resultantes do algoritmo de beamforming clássico em campo distante e campo próximo respectivamente, utilizando-se a geometria da Fig. 5.1. Os eixos para cada caso são diferentes ( $\phi$ e $\theta$ para campo distante e $\mathrm{X}$ e Z para campo próximo) já que em campo próximo é especificado um plano focal sobre o qual é calculada a imagem acústica.

As imagens acústicas apresentadas são as esperadas para este caso simples, certificando que o algoritmo funciona como esperado. Para exemplificar a dificuldade em localizar fontes próximas umas das outras, utiliza-se uma configuração com um número maior de microfones como na Fig. 5.4. Neste experimento, um array de 16 sensores num arranjo retangular com 


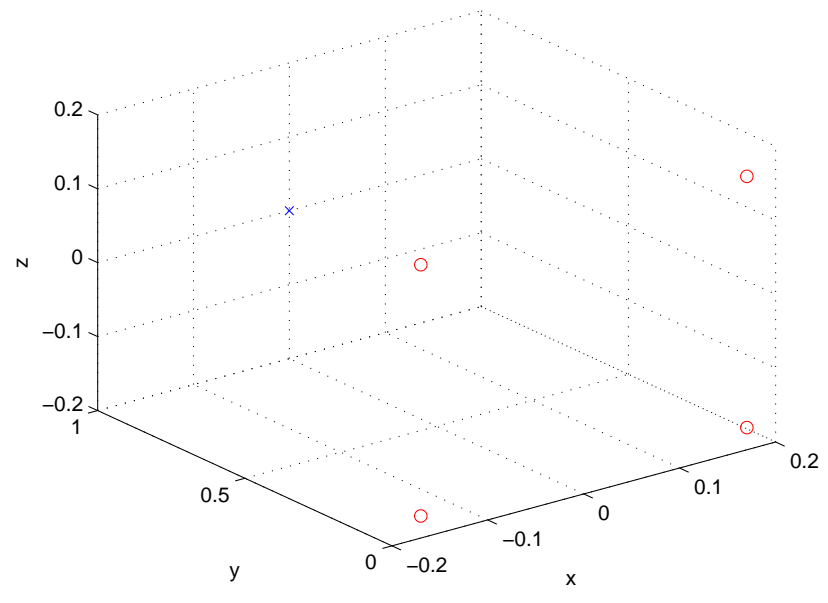

Figura 5.1: Array de 4 sensores (círculos vermelhos) com fonte pontual tonal localizada a 1 metro do plano do array ('x' em azul). Esta configuração básica é utilizada para verificar o funcionamento do algoritmo de beamforming clássico.

Far Field Radiation Pattern

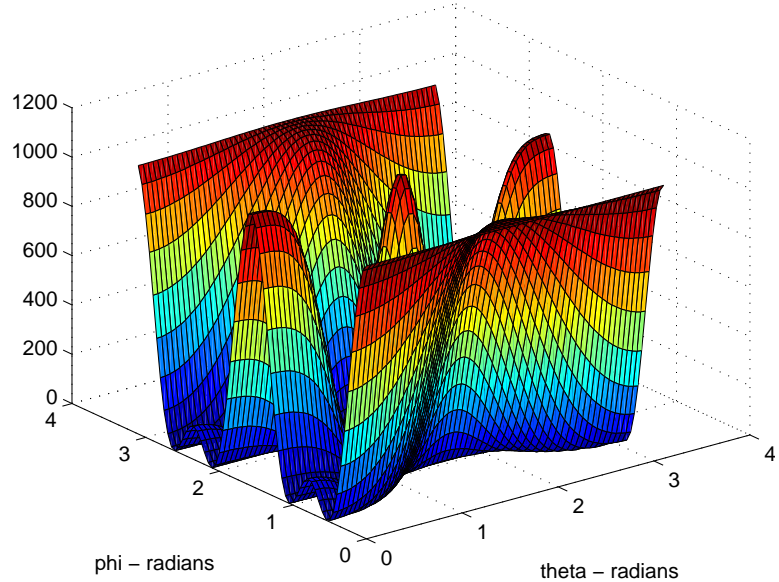

Figura 5.2: Imageamento acústico para uma fonte de $1 \mathrm{KHz}$ posicionada a 1 metro do array em campo distante utilizando o algoritmo de beamforming clássico com 1000 amostras a uma taxa de 40 Kamostras/s. Não há ruído aditivo

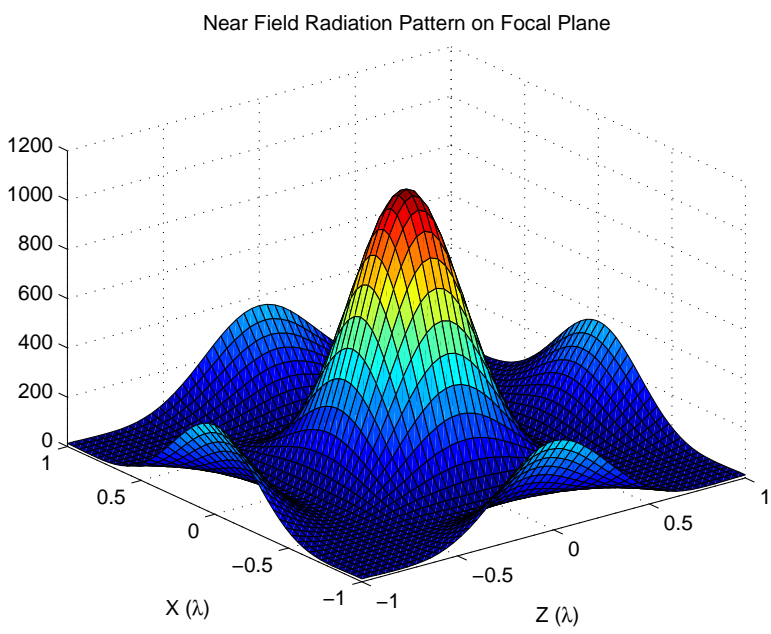

Figura 5.3: Imageamento acústico para uma fonte de $1 \mathrm{KHz}$ posicionada a 1 metro do array em campo próximo utilizando o algoritmo de beamforming clássico com 1000 amostras a uma taxa de 40 Kamostras/s. Não há ruído aditivo

os sensores uniformemente espaçados é utilizado. Colocam-se 2 fontes tonais cada vez mais próximas e determina-se o ponto onde há impossibilidade de localizar as fontes. As Figs. 5.5 e 5.6 ilustram para o campo distante e campo próximo respectivamente a transição para localização de duas fontes. Ocorre o vazamento de potência devido a largura do lóbulo do array, contaminando a estimativa da fonte vizinha. 


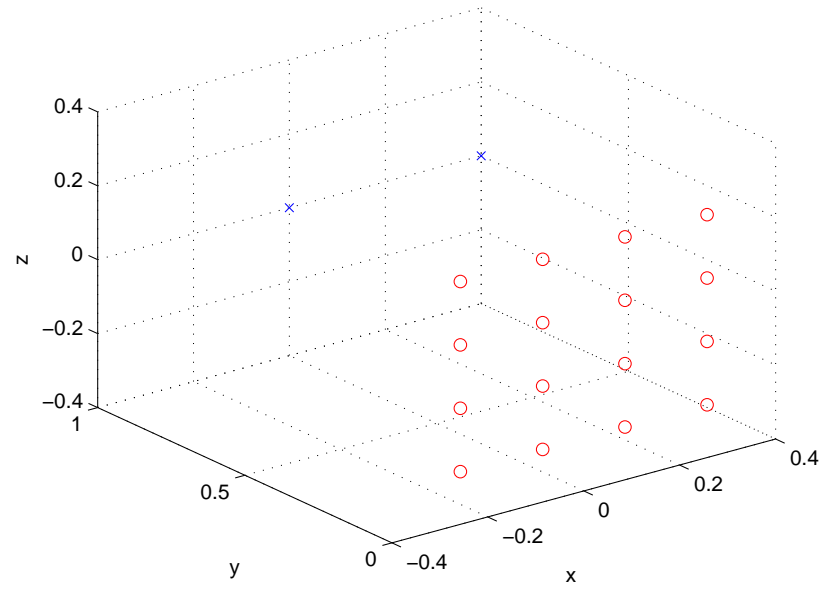

Figura 5.4: Geometria para 2 fontes, uma localizada diretamente a frente e outra deslocada no eixo $X$ por 0.4 metros. O array utilizado é de 16 sensores uniformemente distribuídos num quadrado.

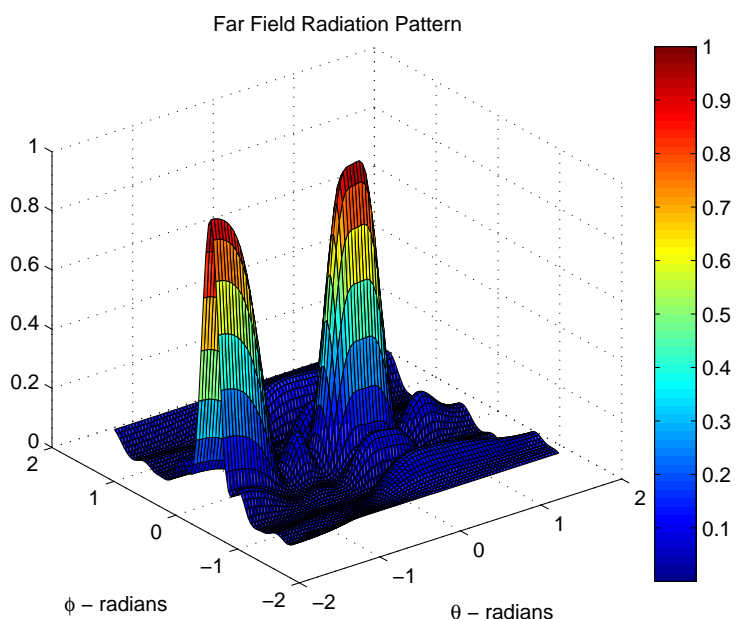

Figura 5.5: Imageamento de campo distante para 2 fontes tonais de $1500 \mathrm{~Hz}$, com 1000 amostras a uma taxa de 40Kamostras/s. As fontes estão localizadas num plano a $1 \mathrm{~m}$ do array, deslocadas entre si por 0.4 no eixo $\mathrm{X}$.

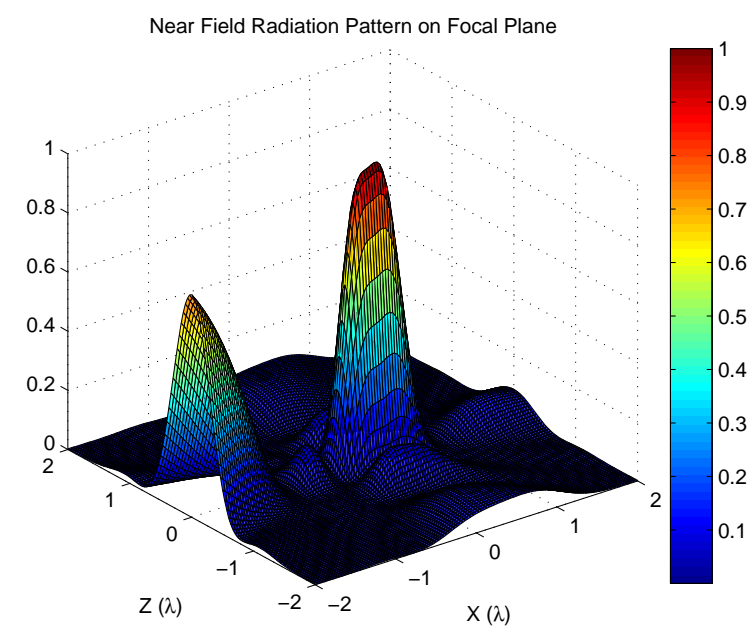

Figura 5.6: Imageamento de campo próximo para 2 fontes tonais de $1500 \mathrm{~Hz}$, com 1000 amostras a uma taxa de 40Kamostras/s. As fontes estão localizadas num plano a $1 \mathrm{~m}$ do array, deslocadas entre si por 0.4 no eixo $\mathrm{X}$.

\subsection{Alta Resolução aplicando 2D-ESPRIT}

Na seção anterior, verificamos apenas o funcionamento do beamforming clássico e analisamos um caso particular sem os efeitos de ruído. Ruído aditivo é incluído na seguinte comparação para simular casos reais. Para evitar a especificação de dois parâmetros separados de potência de ruído e potência do sinal, definimos a razão sinal / ruído como sendo:

$$
S N R=\frac{P_{s}}{\sigma^{2}}
$$


onde $P_{s}$ é a potência do sinal e $\sigma^{2}$ é a potência do ruído.

Os resultados para uma fonte tonal em campo distante para o 2D-ESPRIT e o beamforming clássico, são apresentados nas Figs. 5.7 e 5.8 respectivamente. Ambos utilizaram 800 amostras captadas a uma taxa de 40Kamostras/s de um array quadrado com 16 sensores uniformemente espaçados $(4 \mathrm{x} 4)$. O ruído aditivo manteve uma $\mathrm{SNR}=40 \mathrm{~dB}$. Observa-se que os lóbulos laterais não permitem obter apenas a imagem da fonte com o beamforming clássico, dando a impressão que há mais uma fonte em $(\theta, \phi)=(0.4,-1.2)$. Já para o 2DESPRIT com este array de geometria relativamente simples é capaz de indicar com precisão a localização da fonte.

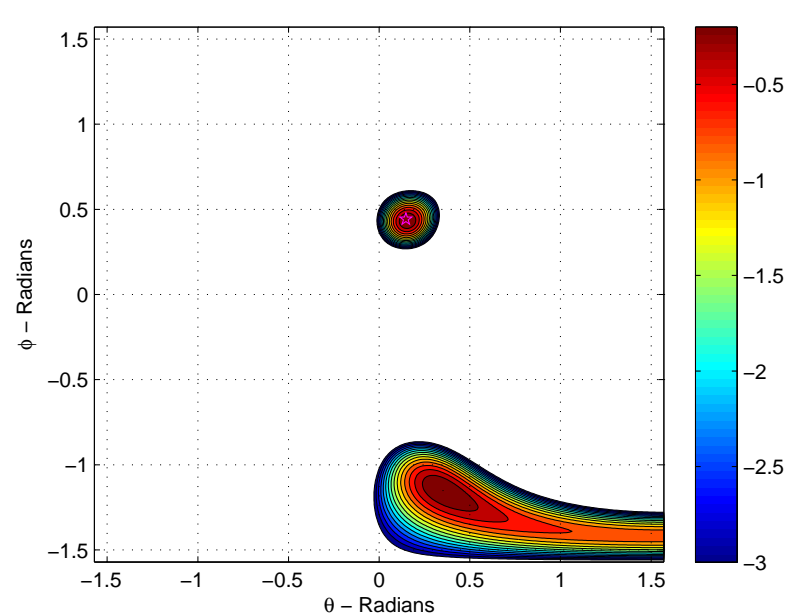

Figura 5.7: Imagem acústica gerada por beamforming clássico para uma fonte de $1500 \mathrm{~Hz}$. A estrela em magenta indica o local verdadeiro da fonte. O array utilizado é quadrado com 16 sensores uniformemente espaçados. 800 amostras a uma taxa de 40Ksamples/s.

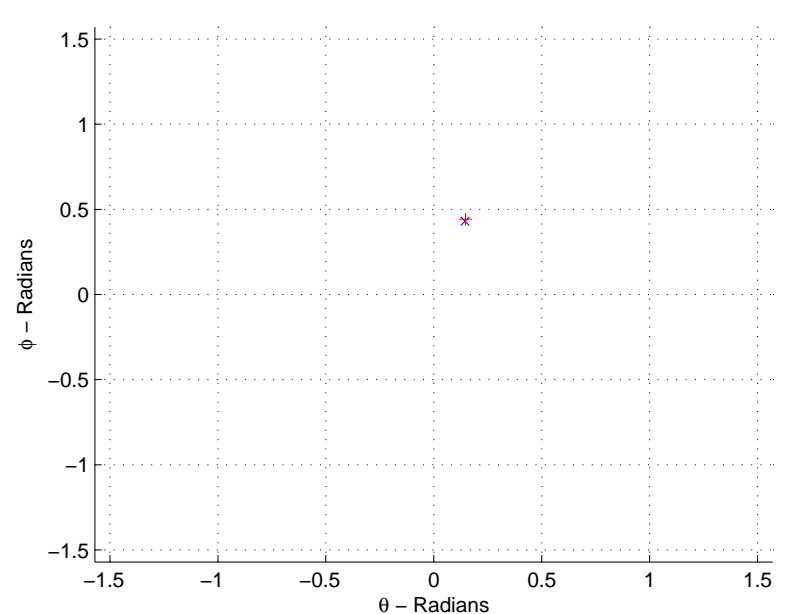

Figura 5.8: Imagem acústica gerada por 2D-ESPRIT para uma fonte de $1500 \mathrm{~Hz}$. A cruz em vermelho indica o local verdadeiro da fonte, enquanto que o ' $x$ ' em azul é a estimativa do 2D-ESPRIT. O array utilizado é quadrado com 16 sensores uniformemente espaçados. 800 amostras a uma taxa de 40Ksamples/s.

Desconsiderando o erro cometido por lóbulos laterais no beamforming clássico, é interessante analisar o erro cometido pelos dois algoritmos para diferentes potências de ruído aditivo. No beamforming clássico, foi considerada a posição do pico da imagem acústica resultante no cálculo do erro de estimativa. A Fig. 5.9 ilustra o comportamento do erro em relação a $S N R$. O erro é calculado em ambos por:

$$
e_{R M S}=\sqrt{(\theta-\hat{\theta})^{2}+(\phi-\hat{\phi})^{2}}
$$

Como pode-se observar o 2D-ESPRIT tem um desempenho sempre melhor do que o beamforming clássico. O 2D-ESPRIT chega a ter aproximadamente $5 \mathrm{~dB}$ de melhora para $S N R=30 \mathrm{~dB}$. Isto se deve a característica de rejeição ao ruído do 2D-ESPRIT ao separar o sinal recebido pelo array em espaço de sinal e espaço de ruído. Esta característica permite 


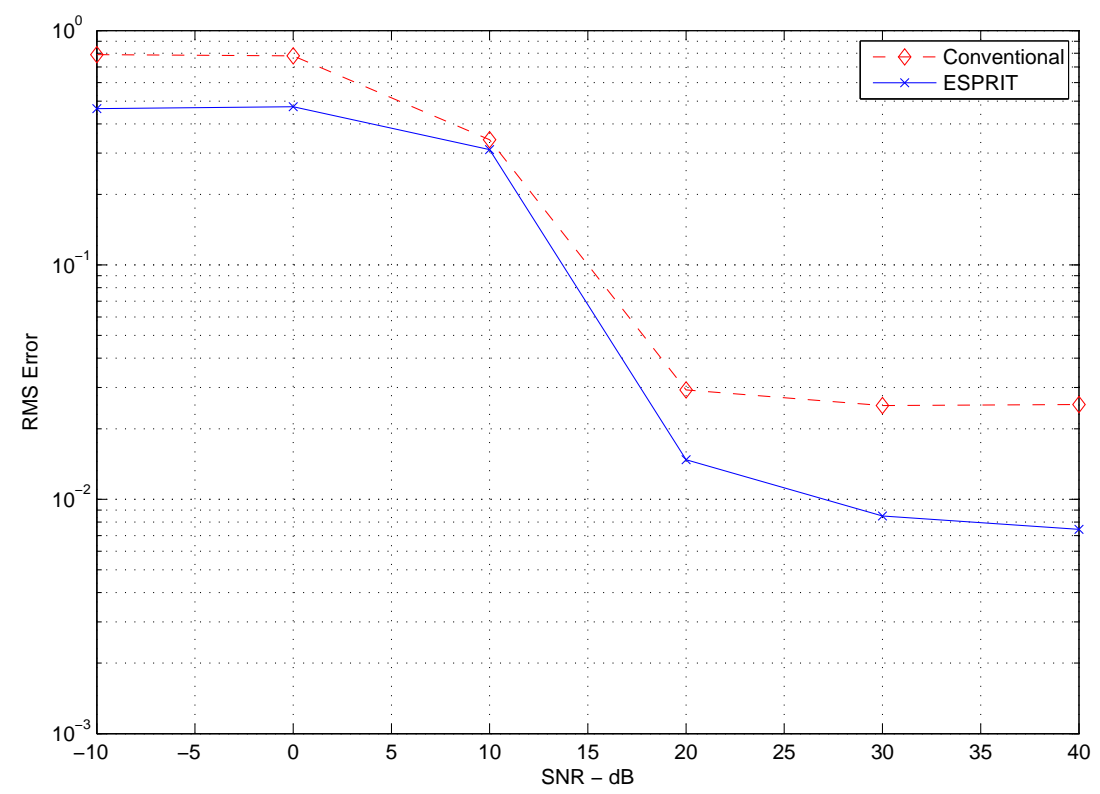

Figura 5.9: Erros de localização para array quadrado 4x4. Foram utilzadas 800 amostras captadas no array de uma fonte tonal pontual localizada no campo distante na frequência de $1500 \mathrm{~Hz}$. Variando a SNR é possível comparar o desempenho do beamforming clássico e do 2D-ESPRIT

que o 2D-ESPRIT não seja somente um algoritmo de alta resolução, mas também retira parte do ruído aditivo presente na medida.

A propriedade de alta resolução do 2D-ESPRIT é verificada com duas fontes pontuais próximas. Considerando o mesmo array anterior de 16 sensores, 800 amostras a uma taxa de 40Kamostras/s, posicionam-se duas fontes de $1500 \mathrm{~Hz}$ próximas uma da outra. As fontes são geradas a partir da filtragem de ruído branco por um filtro estreito em $1500 \mathrm{~Hz}$. O ruído é descorrelacionado para cada fonte, garantindo assim, fontes com mesma frequência mas descorrelacionadas entre si. As Figs. 5.10 e 5.11 apresentam a imagem acústica gerada pelo beamforming clássico e pelo 2D-ESPRIT respectivamente. No beamforming clássico, não é possível determinar a posição das fontes, nem mesmo é possível distinguir a presença de duas fontes. O pico ficou entre as duas fontes que são indicadas pelas estrelas em magenta. O 2D-ESPRIT consegue com precisão identificar as duas fontes e localiza-las corretamente.

A precisão do 2D-ESPRIT se estende para a localização de multiplas fontes. Para exemplificar o seu funcionamento para este caso, são colocadas 4 fontes tonais a $1500 \mathrm{~Hz}$ em diferentes posições. As Figs. 5.12 e 5.13 apresentam a imagem acústica destas fontes para 100 iterações com 800 amostras e 1000 amostras respectivamente. Em ambos os casos as amostras foram colhidas a uma taxa de $40 \mathrm{Kamostras} / \mathrm{s}$ e $S N R=40 \mathrm{~dB}$. Comparando estas duas figuras, o 2D-ESPRIT tem a variância de sua estimativa diminuida com o aumento do número de amostras. Isto é vantajoso, já que pode-se melhorar a precisão colhendo um número maior de amostras. As Figs. 5.14 e 5.15 apresetam o beamforming clássico e o 


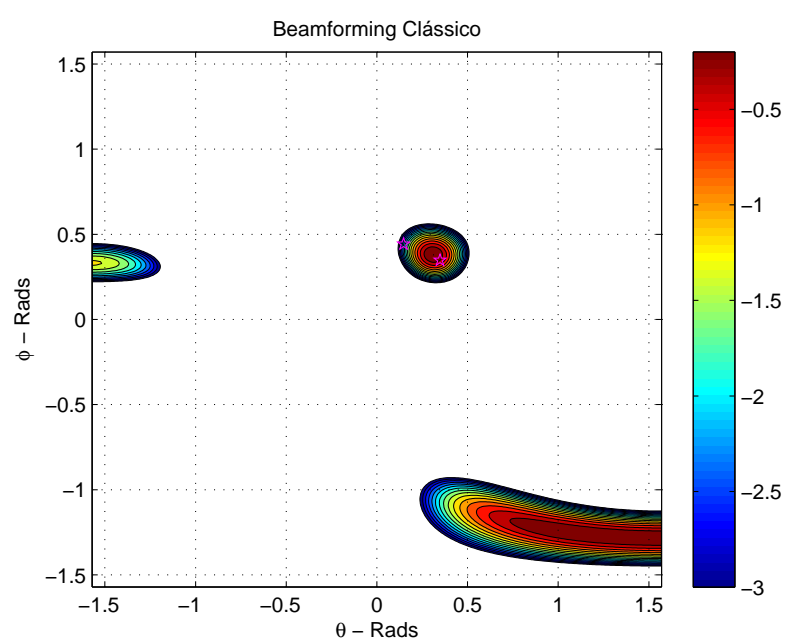

Figura 5.10: Imagem acústica gerada por beamforming clássico para 2 fontes tonais de $1500 \mathrm{~Hz}$. As estrelas em magenta indicam as posições verdadeiras das fontes. O array utilizado é quadrado com 16 sensores uniformemente espaçados. 800 amostras a uma taxa de 40Ksamples/s.

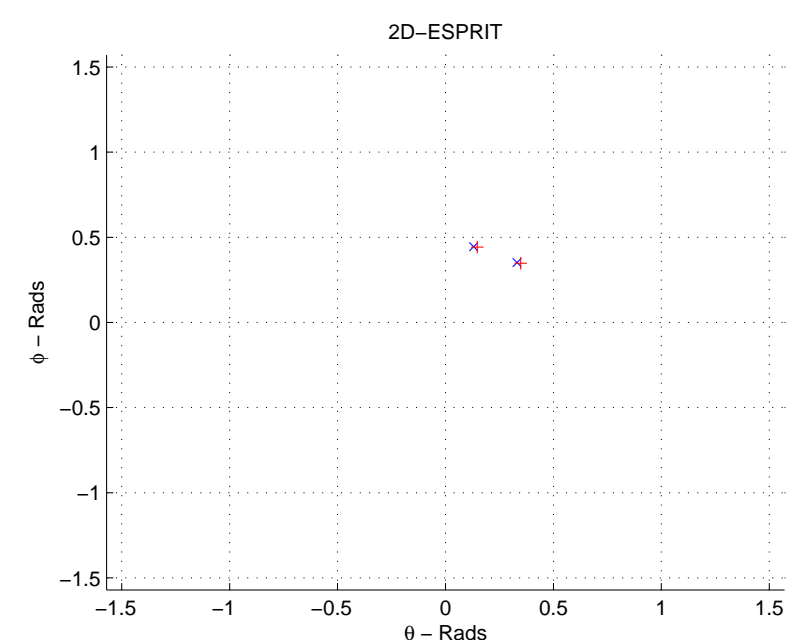

Figura 5.11: Imagem acústica gerada por 2D-ESPRIT para 2 fontes tonais de $1500 \mathrm{~Hz}$. As cruzes em vermelho indicam as posições verdadeiras das fontes, e os ' $\mathrm{x}$ ' em azul indicam as estimativas do 2D-ESPRIT. O array utilizado é quadrado com 16 sensores uniformemente espaçados. 800 amostras a uma taxa de 40Ksamples/s.

2D-ESPRIT respectivamente aplicados as condições de 4 fontes tonais em $1500 \mathrm{~Hz}$ com 1000 amostras com uma taxa de amostragem de 40Kamostras/s e $\mathrm{SNR}=40 \mathrm{~dB}$.

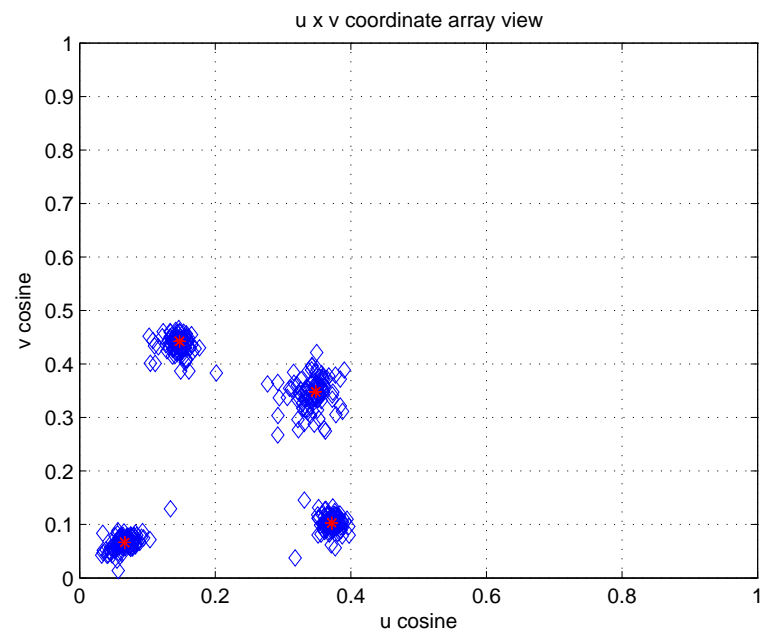

Figura 5.12: Imagem acústica gerada por 2D-ESPRIT com 4 fontes e 800 amostras a uma taxa de $40 \mathrm{Kamostras} / \mathrm{s}$. Todas as fontes são tonais em $1500 \mathrm{~Hz}$. 


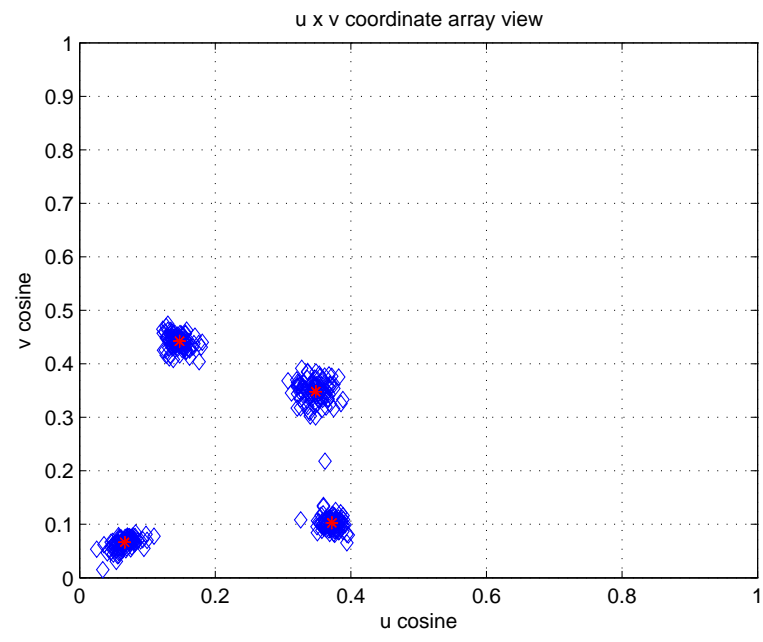

Figura 5.13: Imagem acústica gerada por 2D-ESPRIT com 4 fontes e 1000 amostras a uma taxa de 40Kamostras/s. Todas as fontes são tonais em $1500 \mathrm{~Hz}$.

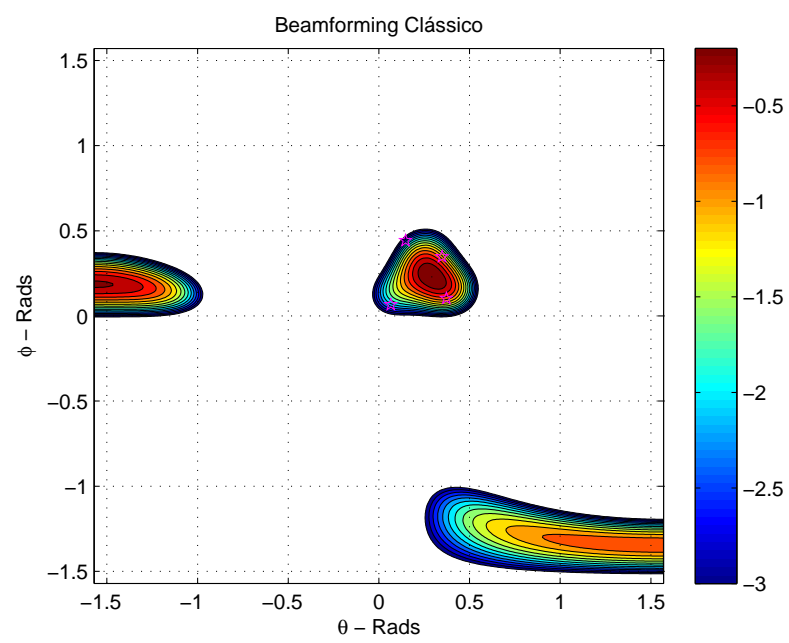

Figura 5.14: Imagem acústica gerada por beamforming clássico com 4 fontes indicadas pelas estrelas em magenta. $\mathrm{O}$ array utilizado é quadrado com 16 sensores uniformemente espaçados. 800 amostras a uma taxa de 40Ksamples/s.

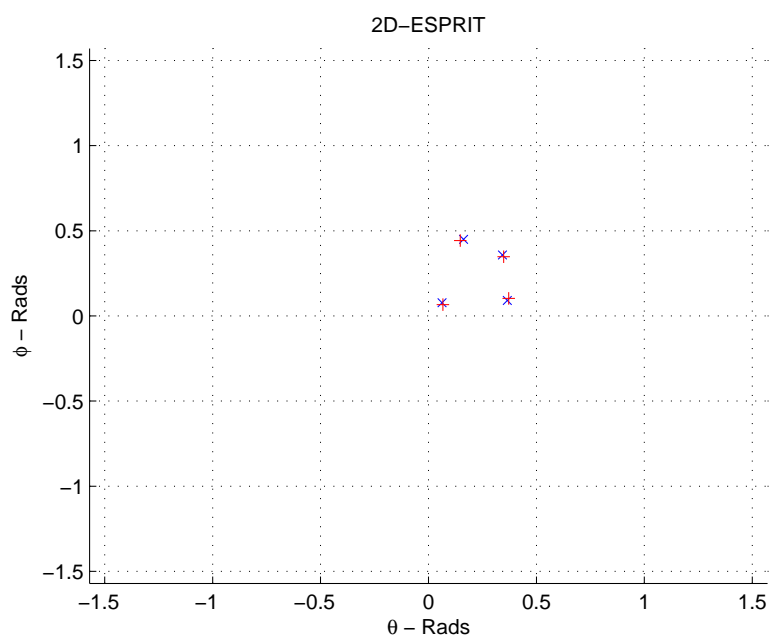

Figura 5.15: Imagem acústica gerada por 2D-ESPRIT com 4 fontes indicadas pelas cruzes vermelhas. Os ' $\mathrm{x}$ ' em vermelho indicam as estimativas do 2D-ESPRIT. O array utilizado é quadrado com 16 sensores uniformemente espaçados. 800 amostras a uma taxa de 40Ksamples/s. 


\section{Capítulo 6}

\section{Conclusões}

$\mathrm{Na}$ análise feita no Cap. 2, os campos distante e próximo definem formas diferentes de localizar as fontes. Em particular, a distância do array até a fonte é um parâmetro adicional a ser determinado quando a fonte está em campo próximo. Isto implica em obter mais informação sobre a fonte em campo próximo do que em campo distante. O campo próximo do array é semelhante ao funcionamento de uma camera fotográfica. Ao focalizar um objeto o conjunto óptico é ajustado para um plano focal localizado a uma certa distância. Em arrays, o "foco" é feito através dos pesos. As fontes acústicas são bem definidas na imagem acústica gerada, porém fontes fora do plano focal estão "fora de foco" e sem boa definição na imagem acústica. Ao utilizar os métodos de processamento de sinais devemos ter o conceito de campo distante versus campo próximo em mente para poder analisar corretamente as imagens acústicas que são geradas.

As imagens acústicas são geradas, numa primeira análise, por beamforming clássico. O beamforming clássico é comumente empregado para determinar a posição de fontes, mas apesar de simples e intuitivo, o beamforming clássico produz imagens acústicas insatisfatórias e que impossibilitam a correta identificação de fontes. A resolução de fontes próximas é limitado pela largura do lóbulo principal do array. Modificando a geometria do array e o número de sensores empregados, ou aplicando ferramentas de estimação espectral como janelamentos e alisamentos espectrais, o beamforming clássico produz bons resultados mas com um custo elevado no projeto do array (i.e. número elevado de microfones e geometrias complexas) e um processamento oneroso (i.e. custo computacional elevado).

O método de alta resolução ESPRIT permite uma melhor localização das fontes, retirando a dependência da largura do lóbulo principal. A implementação mostrou que além da maior resolução obtida com o 2D-ESPRIT, este tem, frente ao ruído de fundo, um desempenho sempre melhor que o beamforming para localizar as fontes de som. Em geral, para o 2D-ESPRIT, o projeto do array é simples e com poucos microfones. O beamforming clássico para ter boa resolução depende da largura do lóbulo principal que por sua vez depende da geometria do array e da quantidade de microfones empregados. Outra vantagem do 2D-ESPRIT é sua baixa carga computacional, já que, a varredura espacial não é feita 
como no beamforming clássico. Ressalta-se, porém, que o 2D-ESPRIT como implementado é limitado a fontes estáticas e em campo distante. Com considerações do efeito Doppler, o método é aplicável a situações de ensaios de "fly-over" onde as fontes presentes no avião em movimento estão no campo distante de um array montado na cabeceira da pista de pouso.

Conclui-se mencionando que o método 2D-ESPRIT é aplicado tanto para localizar as fontes isoladamente, quanto para os métodos de deconvolução. Neste último, o 2D-ESPRIT gera os pontos iniciais para o processo iterativo da deconvolução. Iniciando o processo iterativo na proximidade das fontes pode garantir que a deconvolução irá convergir.

\subsection{Contribuições}

Ds possíveis alternativas de processamento dos dados do array, o beamforming clássico é limitado nos aspectos de resolução e da estimativa da localização quando na presença de ruído aditivo. No entanto, o beamforming clássico é útil numa primeira abordagem ao construir a imagem acústica, desde que observadas suas limitações. Destes estudos e análises resultou o desenvolvimento de software capaz de gerar as imagens acústicas obtidas por um array com qualquer geometria. Dentre os módulos do software de simulação está o gerador de sinais de um array com geometria e número de sensores quaisquer, e no processamento de sinais captados por array foi implementado o beamformer clássico. Estes dois módulos permitem verificar como o beamforming clássico se comporta em diferentes situações.

A estimação espectral paramétrica de alta resolução ESPRIT chamou a atenção para a independência da largura do lóbulo principal do array. Adicionalmente, sua baixa carga computacional e a facilidade adicional de não precisar varrer o espaço para localizar as fontes, despertou interesse em aplicar o ESPRIT no imageamento acústico. No formato original, o ESPRIT é apresentado como um método de alta resolução para arrays lineares. O desafio foi aplicar um método baseado no ESPRIT que trabalhasse nos dados coletados de arrays planares. O 2D-ESPRIT foi implementado para arrays planares, apresentando as características de alta resolução desejadas. O módulo 2D-ESPRIT de processamento de sinais do array é a contribuição feita para implementação do algoritmo. A Fig. 6.1 ilustra os módulos criados em MatLab e a sua aplicação.

Devido a flexibilidade trazida pela modularização dos componentes, é simples utilizar dados captados de arrays nos algoritmos de processamento de sinais para gerar as imagens acústicas ao invés do simulador.

\subsection{Desafios}

O primeiro estudo feito neste trabalho aponta que é mais atraente trabalhar com o array em campo próximo, dada a possibilidade de obter a distância entre fonte e array. A continuidade deste trabalho é implementar um novo método de alta resolução baseado em 


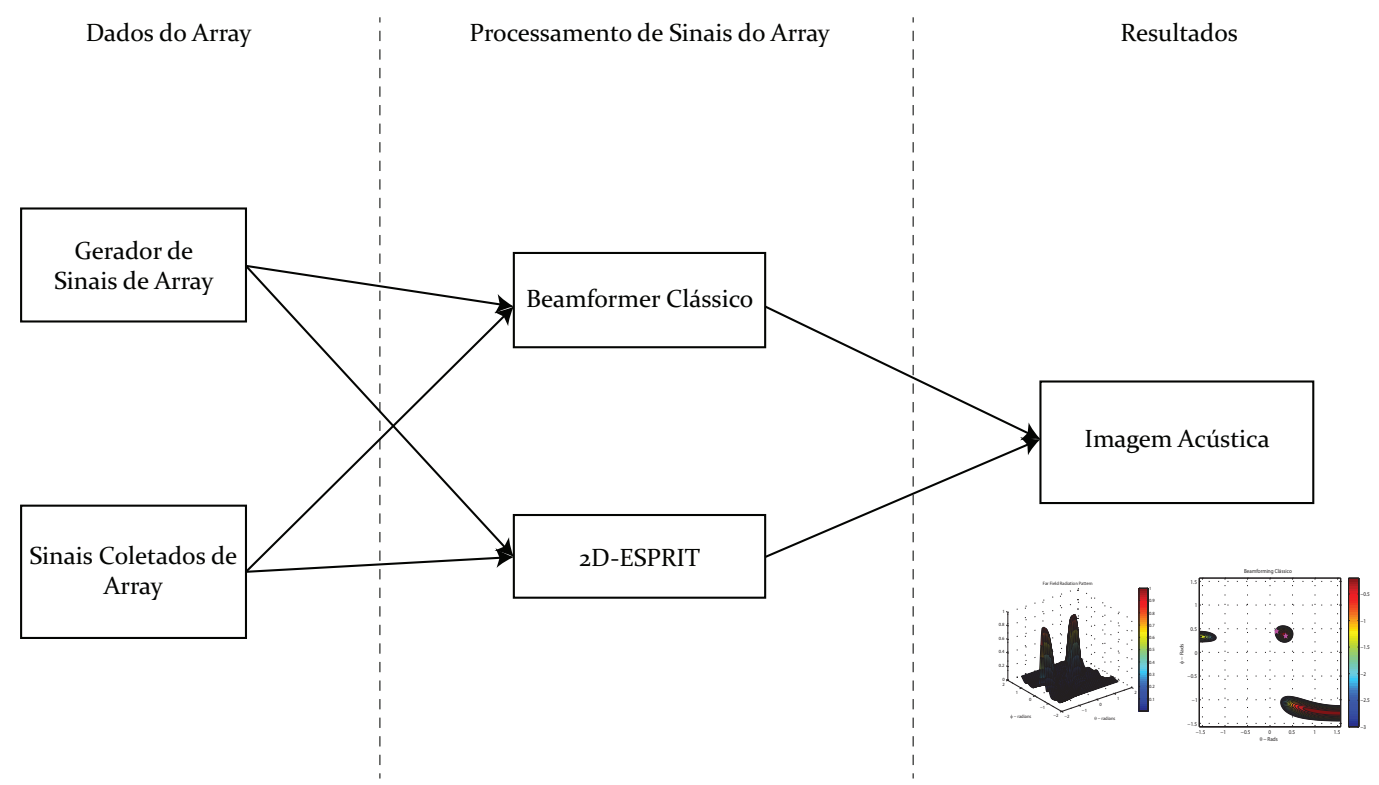

Figura 6.1: Diagrama de uso dos módulos do software desenvolvidos.

ESPRIT para situações onde o array opera em campo próximo. Estão contempladas apenas as fontes estáticas já que estas facilitaram a análise e implementação. Porém, as aplicações em aeroacústicas, especificamente nos testes de "fly-over", há fontes em movimento. Neste caso, é preciso considerar o efeito Doppler e o rastreamento de múltiplas fontes em movimento. Portanto, um segundo objetivo seria aplicar o 2D-ESPRIT e o novo método de alta resolução para estas fontes. Por fim, é desejável obter uma implementação do software desenvolvido neste trabalho num hardware compacto para utilização em campo. O hardware não precisa ser complexo pois, como visto, o 2D-ESPRIT tem baixa carga computacional. Esta solução embarcada forneceria imageamento acústico em tempo real. 


\section{Apêndice A}

\section{Teoria da Difração}

A teoria da difração em óptica estuda o comportamento da propagação da onda eletromagnética quando esta encontra obstáculos. A teoria é generalizada para qualquer propagação ondulatória, como por exemplo a propagação sonora. A difração tem sua melhor descrição através da análise de Fresnel (1818) e a formulação matemática apresentada por Kirchhoff (1882), vide [15] ou [4].

Difrações ocorrem quando uma onda, propagando-se em uma direção qualquer, encontra um obstáculo. O que se percebe é que a onda exibe a propriedade de curvar-se nos cantos de obstáculos. Um experimento simples que verifica o efeito da difração óptica é aquele apresentado na Fig. A.1. A figura ilustra uma fonte $P$ a uma distância suficientemente grande para que as ondas que chegam ao orifício do anteparo sejam planas.

Considerando neste exemplo que a fonte $P$ seja uma fonte de luz, espera-se intuitivamente que numa tela do outro lado do anteparo, apresente uma região central de luz. No entanto, não é este o comportamento que se observa, apresentando um padrão de interferência numa região maior do que a que se espera, ou seja, que a onda de luz dobrou-se nos cantos do orifício.

Para explicar este fenomeno faz-se o uso de dois conceitos: o princípio de Huygens e a interferência de ondas. O princípio de Huygens considera cada ponto de uma frente de onda como sendo uma fonte secundária de ondas de mesmo comprimento de onda que se deslocam com a mesma velocidade. Passado um tempo $\Delta t$, a superfície tangente a estas ondas secundárias é a nova posição da frente de onda. A interferência de ondas explica o comportamento das ondas quando temos mais de uma fonte presente. A Fig. A.2 ilustra o experimento de Young feito com duas fontes coerentes de luz $S_{1}$ e $S_{2}$. No anteparo se observam regiões de máximo e mínimo na intensidade da luz, ou seja, o padrão de interferência. Este padrão se deve as interferências construtivas e destrutivas entre as duas fontes formando respectivamente as regiões de máximo e mínimo na tela. Os tamanhos das regiões de máximo e mínimo dependem do comprimento de onda e da distância das fontes até a tela, já que estes parâmetros indicam o valor das fases da onda de $S_{1}$ e de $S_{2}$ que atingem um ponto $y$ da tela. 
Tela
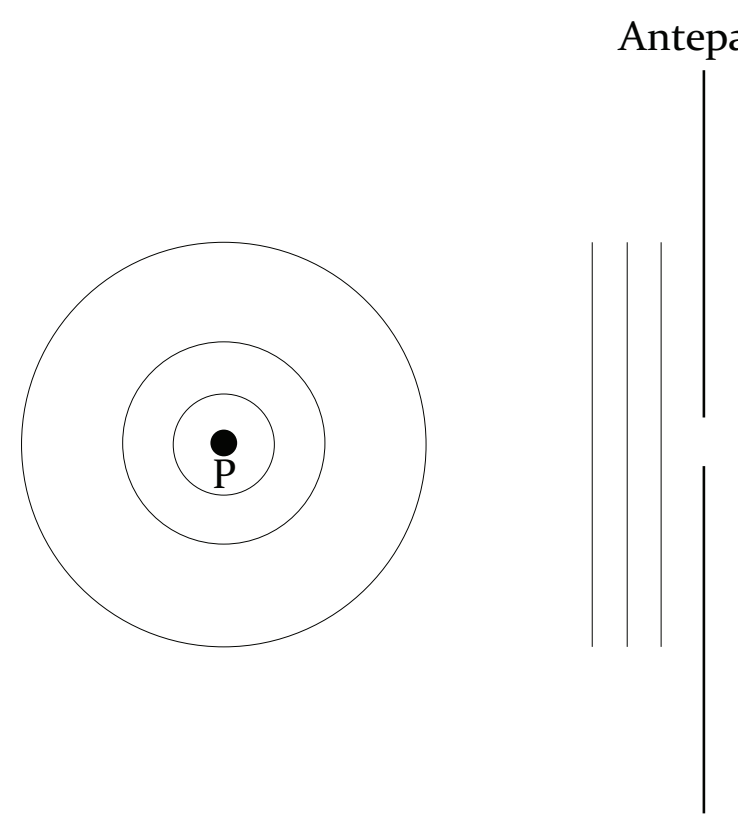

Figura A.1: Condição experimental para observar a difração. Uma fonte monocromática a frente de um anteparo com um furo produz na tela regiões circulares concêntricos iluminadas além de uma região central iluminada.

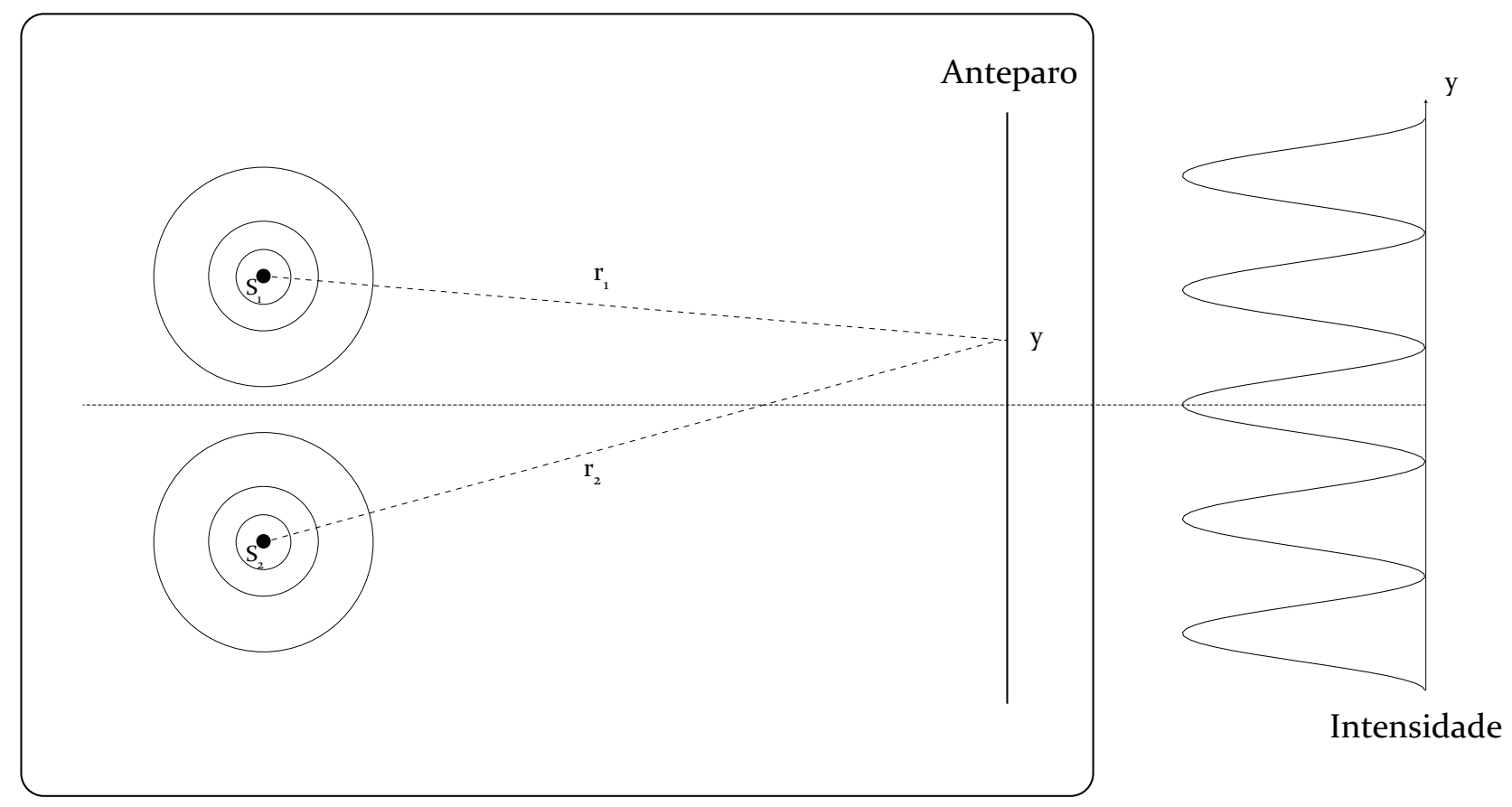

Figura A.2: Experimento de Young para estudo de padrões de interferência. Duas fontes $S_{1}$ e $S_{2}$ distantes $r_{1}$ e $r_{2}$ respectivamente de um anteparo produzem uma padrão de interefência como mostra o gráfico de intensidade luminosa à esquerda. 
Na difração, os dois conceitos são empregados ao dividir o orifício em segmentos na Fig. A.1. Cada segmento do orifício é uma nova fonte da onda que antinge o anteparo, segundo o princípio de Huygens. Aplicando o conceito de interferência de ondas nestas multiplas fontes de ondas no orifício, o padrão de interferência resultante é o que se encontra na tela colocada a frente do anteparo. O formato e as faixas de intensidade máxima e mínima do padrão de interferência, dependem de diversos parâmetros como comprimento de onda, distância entre o anteparo à tela, formato do orifício, etc.

A difração por ser um efeito característico de propagação de ondas, não está restrito apenas à óptica. É na óptica que seu efeito é o mais visível, mas além da obvia extensão para a propagação de ondas eletromagnéticas, a difração é observada também em acústica. 


\section{Referências Bibliográficas}

[1] Constantine A. Balanis. Antenna Theory: Analysis and Design. Wiley-Interscience, 3rd edition, April 2005.

[2] A. Barabell. Improving the resolution performance of eigenstructure-based directionfinding algorithms. In Acoustics, Speech, and Signal Processing, IEEE International Conference on ICASSP '83., volume 8, pages 336 - 339, April 1983.

[3] J. Benesty, J. Chen, and Y. Huang. Microphone Array Signal Processing, volume 1 of Springer Topics in Signal Processing. Springer, March 2008.

[4] M. Born and E. Wolf. Principles of Optics. CambridgeUniversity Press, 1998, 1998.

[5] M. Brandstein and D. Ward, editors. Microphone Arrays: Signal Processing Techniques and Applications (Digital Signal Processing). Springer, 2001.

[6] T. F. Brooks and W. M. Humphreys. A deconvolution approach for the mapping of acoustic sources (damas) determined from phased microphone arrays. In 10th AIAA/CEAS Aeroacoustics Conference. AIAA, 2004.

[7] J. Capon. High-resolution frequency-wavenumber spectrum analysis. Proceedings of the IEEE, 57(8):1408 - 1418, August 1969.

[8] M.P. Clark and L.L. Scharf. Two-dimensional modal analysis based on maximum likelihood. Signal Processing, IEEE Transactions on, 42(6):1443 -1452, June 1994.

[9] Jesus Diaz. Maybe the Most Stupid-Looking Military Invention of All Time, http://gizmodo.com/5153094/maybe-the-most-stupid+looking-military-invention-ofall-time, February 2009.

[10] J. Dmochowski, J. Benesty, and S. Affés. On spatial aliasing in microphone arrays. IEEE TRANSACTIONS ON SIGNAL PROCESSING, 57(4):1383-1395, April 2009.

[11] J.P. Dmochowski and R.A. Goubran. Decoupled beamforming and noise cancellation. IEEE TRANSACTIONS ON INSTRUMENTATION AND MEASUREMENT, 56:8088, 2007. 
[12] Klaus Ehrenfried and Lars Koop. A comparison of iterative deconvolution algorithms for the mapping of acoustic sources. AIAA Journal, 45(7):1584-1594, May 2006.

[13] Jack D. Gaskill. Linear Systems, Fourier Transforms, and Optics (Wiley Series in Pure and Applied Optics). Wiley-Interscience, 1978.

[14] Gene H. Golub and Charles F. Van Loan. Matrix Computations. The John Hopkins University Press, 1996.

[15] Joseph W. Goodman. Introduction to Fourier Optics. Roberts \& Company Publishers, 2004.

[16] M. Haardt, M.D. Zoltowski, C.P. Mathews, and J. Nossek. 2d unitary esprit for efficient 2d parameter estimation. In Acoustics, Speech, and Signal Processing, 1995. ICASSP95., 1995 International Conference on, volume 3, pages 2096 -2099 vol.3, May 1995.

[17] Eugene Hecht. Optics (4th Edition). Addison Wesley, 2001.

[18] J.A. Högbom. Aperture synthesis with a non-regular distribution of interferometer baselines. Astronomy \& Astrophysics Supplement, 15:417-426, June 1974.

[19] William M. Humphreys, Thomas F. Brooks, William W. Hunter, and Kristine R. Meadows. Design and use of microphone directional arrays for aeroacoustic measurements. 36 st Aerospace Sciences Meeting \& Exhibit, Reno NV, pages 98-0471, 1998.

[20] Lawrence E. Kinsler, Austin R. Frey, Alan B. Coppens, and James V. Sanders. Fundamentals of Acoustics. Wiley, 1999.

[21] Anna Lee. Centrohermitian and skew-centrohermitian matrices. Linear Algebra and its Applications, 29:205 - 210, 1980.

[22] M. J. Lighthill. On sound generated aerodynamically. i. general theory. Proceedings of the Royal Society of London. Series A, Mathematical and Physical Sciences, 211(1107):564-587, 1952.

[23] M. J. Lighthill. On sound generated aerodynamically. ii. turbulence as a source of sound. Proceedings of the Royal Society of London. Series A, Mathematical and Physical Sciences, 222(1148):1-32, 1954.

[24] Djamila Mahmoudi. Speech source localization using a multi-resolution technique. Interactive Voice Technology for Telecommunications Applications, 1998. IVTTA '98. Proceedings. 1998 IEEE 4th Workshop, 1:161-165, 1998.

[25] U. Michel, B. Barsikow, J. Helbig, M. Hellmig, and M. Schüttpelz. Flyover noise measurements on landing aircraft with a microphone array. In 4 th AIAA/CEAS Aeroacoustics 
Conference, 1998. 4th AIAA/CEAS Aeroacoustics Conference, Toulouse, France, June 2-4, 1998.

[26] Donald B. Percival and Andrew T. Walden. Spectral Analysis for Physical Applications. Cambridge University Press, 1993.

[27] R. Roy and T. Kailath. Esprit-estimation of signal parameters via rotational invariance techniques. Acoustics, Speech and Signal Processing, IEEE Transactions on, 37(7):984995, Jul 1989.

[28] J A Scales. Theory of seismic imaging, 1995.

[29] R. Schmidt. Multiple emitter location and signal parameter estimation. Antennas and Propagation, IEEE Transactions on, 34(3):276 - 280, March 1986.

[30] Petre Stoica and Randolph Moses. Spectral Analysis of Signals. Prentice Hall, 1st edition, 2005.

[31] A.L. Swindlehurst, B. Ottersten, R. Roy, and T. Kailath. Multiple invariance esprit. Signal Processing, IEEE Transactions on, 40(4):867 -881, April 1992.

[32] A.J. van der Veen, P.B. Ober, and E.F. Deprettere. Azimuth and elevation computation in high resolution doa estimation. Signal Processing, IEEE Transactions on, 40(7):1828 -1832 , July 1992.

[33] B.D. Van Veen and K.M. Buckley. Beamforming: a versatile approach to spatial filtering. ASSP Magazine, IEEE, 5(2):4 -24, april 1988. 